Check for updates

Cite this: Mater. Adv., 2021, 2,6213

Received 14th April 2021, Accepted 26th August 2021

DOI: 10.1039/d1ma00341k

rsc.li/materials-advances

\section{Optical chemosensors for the gas phase detection of aldehydes: mechanism, material design, and application}

\author{
Chenglong Liao, (D) a Jiangfan Shi, (DD ${ }^{\mathrm{b}}$ Miao Zhang, (D) ${ }^{\mathrm{a}}$ Rana Dalapati, (DD ${ }^{\mathrm{b}}$ \\ Qingyun Tian, (D) ${ }^{a}$ Shuai Chen, (D)*bc Chuanyi Wang (D) ${ }^{a}$ and Ling Zang (D)*b
}

\begin{abstract}
Because of their high hazards and presence in various common scenarios (e.g., workplace, residence, or bio-conversion systems), aldehydes have attracted enormous attention in the research and development of chemical sensors and other detection methods that can provide the quick and sensitive detection of aldehydes, particularly in the gas phase, thus enabling the real-time monitoring of air pollution and impact to health and environment. Among all the detection methods, an optical chemosensor, which relies on aldehyde-triggered color or fluorescence emission change, is typically small in size, easy to operate, and low cost, offering great advantages over benchtop spectrophotometric or chromatographic instrumentation methods, which are large in size and non-portable, complicated to operate, and expensive to maintain. This review will provide a comprehensive overview of the most remarkable development in the gas phase detection of aldehydes (especially the highly volatile aliphatic aldehydes such as formaldehyde and acetaldehyde) in recent years, mostly focusing on the colorimetric, fluorometric, colorimetric/fluorometric dual-mode, and chemiluminescent sensing methods. The review is specifically centered on the discussion of the sensor mechanism in correlation with various molecular designs and materials structural engineering, which, when combined, would lead to maximum sensor performance with respect to both the detection sensitivity and selectivity. At the end of this paper, we also provide the future perspectives concerned with the future development of optical chemosensors and miniaturized device systems, which will be more suited for deployment in practical applications for the quick and onsite detection of aldehydes.
\end{abstract}

${ }^{a}$ School of Environmental Science and Engineering, Shaanxi University of Science and Technology, Xi'an 710021, China

${ }^{b}$ Nano Institute of Utah and Department of Materials Science and Engineering, University of Utah, Salt Lake City 84112, Utah, USA. E-mail: shuaichen@jxstnu.edu.cn, lzang@eng.utah.edu; Fax: +1-801-581-4816; Tel: +1-801-587-1551

${ }^{c}$ Flexible Electronics Innovation Institute, Jiangxi Science \& Technology Normal University, Nanchang 330013, Jiangxi, China

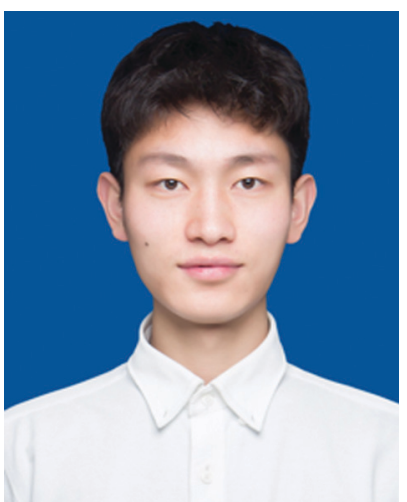

Chenglong Liao
Chenglong Liao graduated from Henan University of Urban Construction (China) in 2019 with a major in environmental science. After that, he has been studying as a master student at Shaanxi University of Science and Technology (China) under the supervision of Prof. Ling Zang and Prof. Shuai Chen. His research is focused on the realtime detection of formaldehyde in the gas phase by optical chemosensor methods.

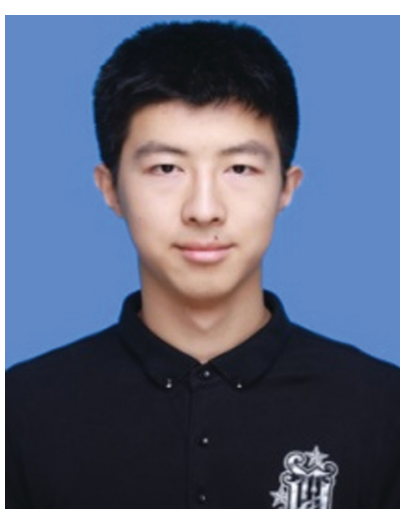

Jiangfan Shi graduated with a BS degree in materials science and engineering from Hunan University, China, in 2020. He is now a PhD student under the supervision of Prof. Ling Zang at the University of Utah. His current research focuses on functional organic nanomaterials as well as their applications in chemical sensors.

Jiangfan Shi 


\section{Introduction}

Aldehydes (R-CHO) are typically colorless, chemically active, and can be formed from the release or decomposition of chemical additives and industrial processes, food fermentation, ripening of fruits, and other sources. Thus, they are found widely in the environment, industrial chemicals, alcohols, as well as human metabolites. ${ }^{1-5}$ Small molecule aldehydes are volatile and can be leaked into air, causing pollution and health hazards. Direct or indirect exposure to gaseous aldehydes can cause various healthrelated issues, such as skin and eye irritation in low concentration contact, central nervous system damage, immune system lesion, blindness, and respiratory dysfunction when higher concentration of aldehydes are inhaled. ${ }^{6-9}$ Aldehydes are also categorized as human carcinogens. For example, there has been explicit epidemiological evidence that exposure to aldehydes can cause nasopharyngeal carcinoma as reported by the International Agency for Research on Cancer. ${ }^{10}$ Moreover, volatile aldehydes have also been identified as biomarkers for many cancers, which are particularly present in exhaled breath. This has led to extensive research on breath analysis with the aim to develop noninvasive diagnosis of diseases simply by detecting aldehydes and other biomarkers such as volatile organic compounds (VOCs) in exhaled breath. Indeed, some aldehydes (e.g., malondialdehyde) have already been used as biomarkers for identifying lung cancer. $^{11-13}$

Among all the aldehydes, the two smallest and most volatile ones, formaldehyde ( $\mathrm{HCHO}, \mathrm{FA})$ and acetaldehyde $\left(\mathrm{CH}_{3} \mathrm{CHO}\right.$, $\mathrm{AA}$, represent the most common and most widely spread indoor air pollutants, causing enormous hazards and health threat. It is no surprise that FA and AA have been studied the most as the target analytes representing aldehydes in the

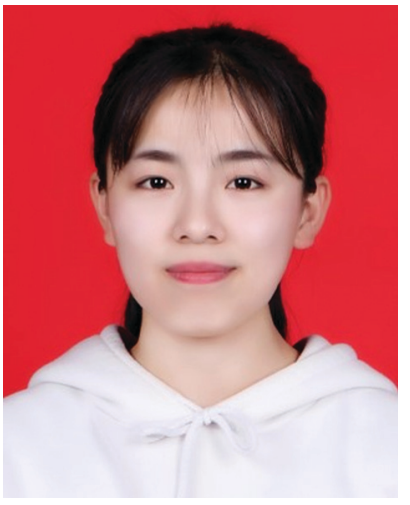

Miao Zhang
Miao Zhang graduated with a major in environmental science from Shanxi Agriculture University, China, in 2019. She is now a master student under the supervision of Prof. Ling Zang and Prof. Shuai Chen at Shaanxi University of Science and Technology, China. Her research is focused on the development of thin-film solid phase microextraction modified with fluorescence chemosensors and its characterization for the trace level detection of water pollutants, particularly organic amines and drugs.

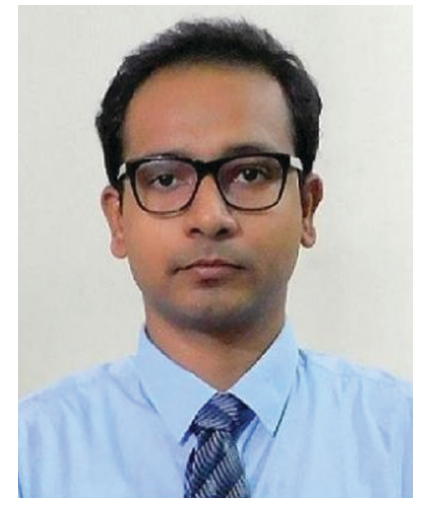

Rana Dalapati
Rana Dalapati got his PhD in Chemistry in 2019 from Indian Institute of Technology, Guwahati, India, under the supervision of Prof. Shyam Prosad Biswas. He is currently a postdoctoral research associate in the group of Prof. Ling Zang at the University of Utah. His major research interests are focused on the design and development of novel MOF structures and their potential application in chemosensors.

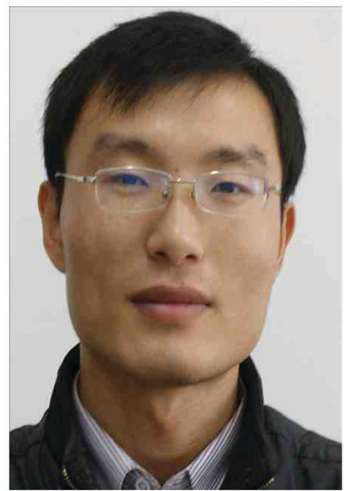

Shuai Chen
Shuai Chen is a professor at Jiangxi Science \& Technology Normal University, China. He earned his PhD degree in materials physics and chemistry from the University of Chinese Academy of Sciences in 2015. He was a postdoctoral fellow with Prof. Ling Zang at University of Utah during 2018-2020. His current research emphasizes the design and fabrication of new organic semiconductor nanocomposites for chemosensors and clean energy materials, and intrinsic conducting polymer films with flexibility and self-healing capability for optoelectronic applications.

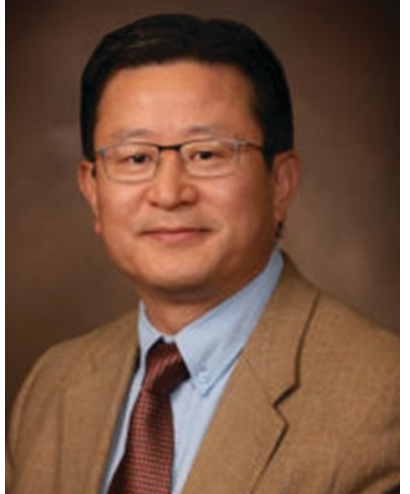

Ling Zang is a professor at the University of Utah. He is also an adjunct professor at Shaanxi University of Science and Technology. He received his $B S$ in chemistry from Tsinghua University and PhD in chemistry from the Chinese Academy of Sciences. He is a Fellow of the National Academy of Inventors (NAI), and was previously an Alexander von Humboldt Fellow, NSF CAREER Award winner, and K. C. Wong Foundation Research Fellow. His current research focuses on nanoscale imaging and molecular probing, organic semiconductor nanostructures, optoelectronic sensors, and nanodevices, with the long-term goal to achieve real applications in the areas of public safety, health monitoring, renewable energy, and clean environment. 
development of chemosensors, especially in gas phase detection. The World Health Organization (WHO) has guidelines to determine that the safe level of exposure to FA in indoor air must not exceed $80 \mathrm{ppb}$ over $30 \mathrm{~min}$, and the Occupational Safety and Health Administration (OSHA) has set the permissible exposure limit at 750 ppb. ${ }^{14}$ For AA, WHO has set the allowable level of exposure concentration to be $<5 \mathrm{ppb}$ and the permissible exposure limit by OSHA is $200 \mathrm{ppm}^{15}$

It has thereby become increasingly important and urgent to develop gaseous sensors to detect aldehydes at a low concentration level, which may find applications in a variety of areas such as environmental monitoring, biomedical analysis, pharmaceuticals, and food industries. ${ }^{16,17}$ Although numerous instrumental analysis methods such as spectrophotometry, ${ }^{18}$ gas chromatography, ${ }^{19}$ high-performance liquid chromatography, ${ }^{20}$ and other techniques derived therefrom have been successfully used in the measurement and analysis of aldehydes in all kinds of samples, these benchtop instrumentations are off-line and based on expensive, complicated apparatus that require long operation time and professionally trained users. Some detection methods, such as semiconductor film sensors, ${ }^{21}$ surface acoustic wave sensors, ${ }^{22}$ and chemiresistive sensors ${ }^{23}$ are much easier to use and can afford on-line detection; however, these sensors often suffer from poor selectivity (against interferences) and low sensitivity (with detection limits usually $>300 \mathrm{ppb}$ ). Alternatively, electrochemical sensors have been extensively used in water and bio-sample analysis due to their simple setup and operation. However, these systems can hardly be adapted into gas phase detection while still maintaining the same level of sensitivity as in the liquid phase simply because it takes extra effort to collect and concentrate the gaseous analytes in the liquid electrolyte where the interfacial electrode reaction occurs. Some research aims to develop all-solid phase electrochemical sensors that allow for the direct detection of gas species through gas phase surface reactions on the electrode, though these electrochemical gas sensors usually suffer from a low efficiency of detection because of the low absorption of gaseous analytes on the electrode surface and low ionic conductivity in the solid phase electrolyte (compared to the liquid phase). To overcome these basic technical limitations, increasing the research efforts has been employed for the development of optical chemosensors that will potentially provide real-time, onsite detection of aldehydes with both high sensitivity and selectivity, and moreover, at low cost and with a small size (portability). ${ }^{24-26}$ Optical chemosensors are based on either color change or fluorescence emission modulation (regarding intensity or wavelength change), which are triggered by chemical interaction between the sensor molecules and aldehydes. One such chemical interaction is aldimine condensation. Both the colorimetric and fluorometric sensors, as well as the colorimetric/fluorometric dual-mode sensors, are suited for the gas phase detection of aldehydes, taking advantages of the vast options of molecular design and structural optimization, which in turn would lead to maximum sensor performance (for high detection sensitivity and selectivity) and simplification or miniaturization of the detection system. In some cases of colorimetric sensors, detection can even be realized with naked eyes but only down to the detection limit as low as ppm-ppb. Moreover, the molecular and material design rules developed from the optical chemosensors for aldehydes can also be adapted to the sensor development for other VOCs as well as the biological analytes in the liquid phase. ${ }^{27-29}$

So far, several excellent reviews have been published focusing on optical chemosensors for FA detection. On the one hand, in view of the requirement of developing portable and real-time analyzers as well as the critical pre-concentration process before analysis, Allouch et al. (2013) focused on the comparison of two different technical routes for gaseous FA trapping (surface adsorption of the doped adsorbent in the solid substrate or uptake into the aqueous reactant) in correlation with the corresponding setup of the optical detection system. ${ }^{24}$ On the other hand, Lee et al. (2013) reviewed the operational principles and sensing performances of real-time and microscale FA gas sensors under the categories of "receptor-" or "transducer-" types, for which several examples on colorimetric detection have been presented. ${ }^{25} \mathrm{Hu}$ et al. (2017) summarized the advances of FA-responsive fluorescent probes for biological applications, with major focus on the different reaction mechanisms and the related design of small recognition molecules. ${ }^{26}$ More recently, Liu et al. (2020) gave special attention to the research progress of fluorescent probes based on organic small-molecule dyes with the amino group, which are capable of detecting three carbonyl species including FA, and enabling solution-phase bioimaging. ${ }^{30}$ Although these reviews cover the current technical status of the detection of FA well, it remains crucial to provide a more comprehensive review to cover broader sensing mechanisms in correlation with the unique molecular designs, which can potentially expand the detection scope from FA to many other aldehydes.

In this paper, we provide a comprehensive review on the recent developments of optical chemosensors, including the colorimetric, fluorometric, and chemiluminescent sensing modes, which have all proven to be effective for the gas phase detection of FA and other aldehydes. Varying sensing mechanisms are covered, including the colorimetric and fluorometric response caused by aldehyde-triggered reactions such as aldimine condensation and Tollens' reaction-mediated localized surface plasmon resonance (LSPR). The development of new sensors based on the microstructural regulation of novel materials such as metal-organic frameworks (MOFs) and quantum dots (QDs) has also been reviewed. Accordingly, the diverse organic and inorganic chromophores of optical chemosensors, as well as their tunable structures and architectures, have also been systematically discussed. Finally, the different sensors are compared for their performance in real applications, which are also correlated with the different sensing mechanisms and membrane substrate materials used, with the aim of providing a reasonable assessment of the current challenges in the gas phase detection of aldehydes, and more importantly, offer some perspectives for the future development of new sensors to further improve the detection sensitivity and selectivity. 


\section{Colorimetric sensors}

Colorimetric chemosensors are becoming more popular due to their highly sensitive and selective response toward gaseous analytes in real-time for onsite detection. ${ }^{31-33}$ The colorimetric signal change can be qualitatively recognized by naked eyes without using any professional equipment and complicated data analysis, which is very useful for the detection of indoor and outdoor aldehydes in a less complicated way. The signal change of the colorimetric system can be quantitatively measured and analyzed via UV-Vis absorption for transparent substrates or reflection for non-transparent substrates, including surface plasmon resonance absorption and light scattering for metal nanostructures. Such a change in the signal can be measured when the sensor molecules interact with aldehyde molecules under appropriate conditions. ${ }^{34-36}$

Based on different types of colorimetric signals due to their different chemical reaction-based sensing mechanisms, the sensors can be roughly divided into five categories, which include aldimine condensation-based sensors, aldimine reactionmediated biomimetic nanostructure tailoring sensor, Hantzsch reaction-based sensor, Sawicki reaction-based sensor, and Tollens' reaction-mediated sensor. A summary and comparison about these sensing mechanisms and the corresponding sensing probe materials, substrates, as well as their sensing performances (selectivity, low detection limit (LDL), response time, etc.) are discussed in the flowing section in line with a brief list in Table 1.

\subsection{Sensors based on aldimine condensation}

The synthesized organic dyes are generally used as chromophore materials for colorimetric chemosensing. Due to the high sensitivity and selectivity, easy synthesis and storage, and excellent stability toward under experimental conditions, reactionbased sensor materials are extensively used by researchers in environmental analysis. ${ }^{37-40}$ Aldimine condensation is a classical addition-elimination reaction based on a nucleophilic addition reaction between the carbonyl group $(-\mathrm{C}=\mathrm{O})$ of the aldehyde molecule and an amine group $\left(-\mathrm{NH}_{2}\right)$ toward the formation of hydrazone, with the loss of one molecule of water $\left(\mathrm{H}_{2} \mathrm{O}\right)$. According to the direct or indirect source of the colorimetric signal, we can classify sensor materials involving the aldehyde-responsive aldimine condensation reaction into three types-hydroxylamine acid salts together with $\mathrm{pH}$ indicators, Schiff reagents, and amine-modified organic chromophores. Moreover, the latter can be further divided into 2,4-dinitrophenylhydrazine (DNPH) and 4-amino-3-hydrazino-5-mercapto1,2,4-triazole (AHMT).

2.1.1 Mixture of hydroxylamine acid salt and $\mathrm{pH}$ indicator. Benefitting from their easy availability and low cost, a mixture of hydroxylamine acid salt and $\mathrm{pH}$ indicator has been used for the indirect colorimetric sensing of aldehydes. The reactions can be divided into two steps. First, the specific aldimine condensation reaction occurs between an amine group $\left(-\mathrm{NH}_{2}\right)$ and an aldehyde group ( $-\mathrm{CHO})$ to generate a Schiff base ${ }^{41}$ by releasing acid ( $\mathrm{HX} ; \mathrm{X}=\mathrm{Cl}, \mathrm{HSO}_{4}$, etc.) in the hydroxylamine salt (eqn (1)). In the second step, the generated acid causes the color change of the $\mathrm{pH}$ indicators such as methyl yellow (MY), methyl red (MR), bromocresol purple (BCP), 4-nitrophenol (4-NP), and rose anthocyanin (RA) in the aqueous phase. ${ }^{42-46}$ The color changes of the $\mathrm{pH}$ indicators arise from their structural change before and after their protonation, as shown in Fig. 1.

Table 1 The summary and comparison of colorimetric chemosensors for gaseous aldehyde detection

\begin{tabular}{|c|c|c|c|c|}
\hline Probing material & Aldehyde & LDL (ppb) & Response time (min) & Ref. \\
\hline Cellulose tape doped with $\left(\mathrm{NH}_{2} \mathrm{OH}\right)_{2} \cdot \mathrm{H}_{2} \mathrm{SO}_{4}$ and $\mathrm{MY}$ & FA & 100 & 30 & 42 \\
\hline Silica-gel cellulose tape doped $\left(\mathrm{NH}_{2} \mathrm{OH}\right)_{2} \cdot \mathrm{H}_{2} \mathrm{SO}_{4}$ and $\mathrm{MY}$ & FA & 80 & 30 & 43 \\
\hline Nylon 6 NFN membrane doped with $\left(\mathrm{NH}_{2} \mathrm{OH}\right)_{2} \cdot \mathrm{H}_{2} \mathrm{SO}_{4}, \mathrm{MY}$ & FA & 50 & 30 & 44 \\
\hline Ormosil coating with $\left(\mathrm{NH}_{2} \mathrm{OH}\right)_{2} \cdot \mathrm{H}_{2} \mathrm{SO}_{4}$ and RA & FA & 60 & 5 & 45 \\
\hline PSMA/PEI with pH indicators & FA & 500 & 1 & 46 \\
\hline Porous glass with Schiff's reagent & FA & 10 & 60 & 51 \\
\hline Electrospun cellulose membrane with Schiff's reagent & FA & 40 & 15 & 52 \\
\hline Glass filter with potassium hydroxide solution and AHMT & FA & 40 & 3 & 54 \\
\hline Finger-type OPD with an SMD LED and $\mathrm{KOH}$ and AHMT & FA & 40 & 20 & 55 \\
\hline Microfluidic chip with AHMT dissolved $\mathrm{HCl}$ and $\mathrm{KOH}$ & FA & 10 & 18 & 56 \\
\hline DNPH and dye & C1-C7 aldehydes & $40(\mathrm{FA})$ & 4 (FA) & 61 \\
\hline \multirow[t]{3}{*}{ CLCs doped with dodecylamine } & Pentyl aldehyde & 2770 & - & 67 \\
\hline & Butyl aldehyde & 4990 & & \\
\hline & Methyl aldehyde & 8502 & & \\
\hline CNCs films with amine groups & Aldehydes & - & - & 68 \\
\hline LRPAs & Aldehydes & - & - & 69 \\
\hline Porous glass impregnated $\beta$-diketone & FA & 14 & $\geq 120$ & 79 \\
\hline 1-Phenyl-1,3-butanedione portable device & FA & 3.5 & 60 & 80 \\
\hline Porous glass impregnated $\beta$-diketone device & FA & 30 & $\geq 60$ & 81 \\
\hline Silica-based hybrid films with Fluoral-p & FA & 60 & 120 & 84 \\
\hline Fluoral-p functionalized PAN & FA & 40 & 30 & 87 \\
\hline Cellulose filter paper doped with KD-XA02 & FA & 5 & 15 & 85 \\
\hline Ag-NPs on ATP-mediated Nafion Film & FA & 60 & 60 & 90 \\
\hline AgNCs@Tollens chemodosimeter & FA & 840 & - & 91 \\
\hline Au@Ag core-shell NPs & FA & 1.5 & - & 92 \\
\hline Au@Ag core-shell NRs with CTAB & FA & 47 & 15 & 93 \\
\hline Au@Ag core-shell NRs & FA & 0.2 & - & 94 \\
\hline Au NPs array & Aldehydes & $900(\mathrm{FA})$ & - & 95 \\
\hline
\end{tabular}



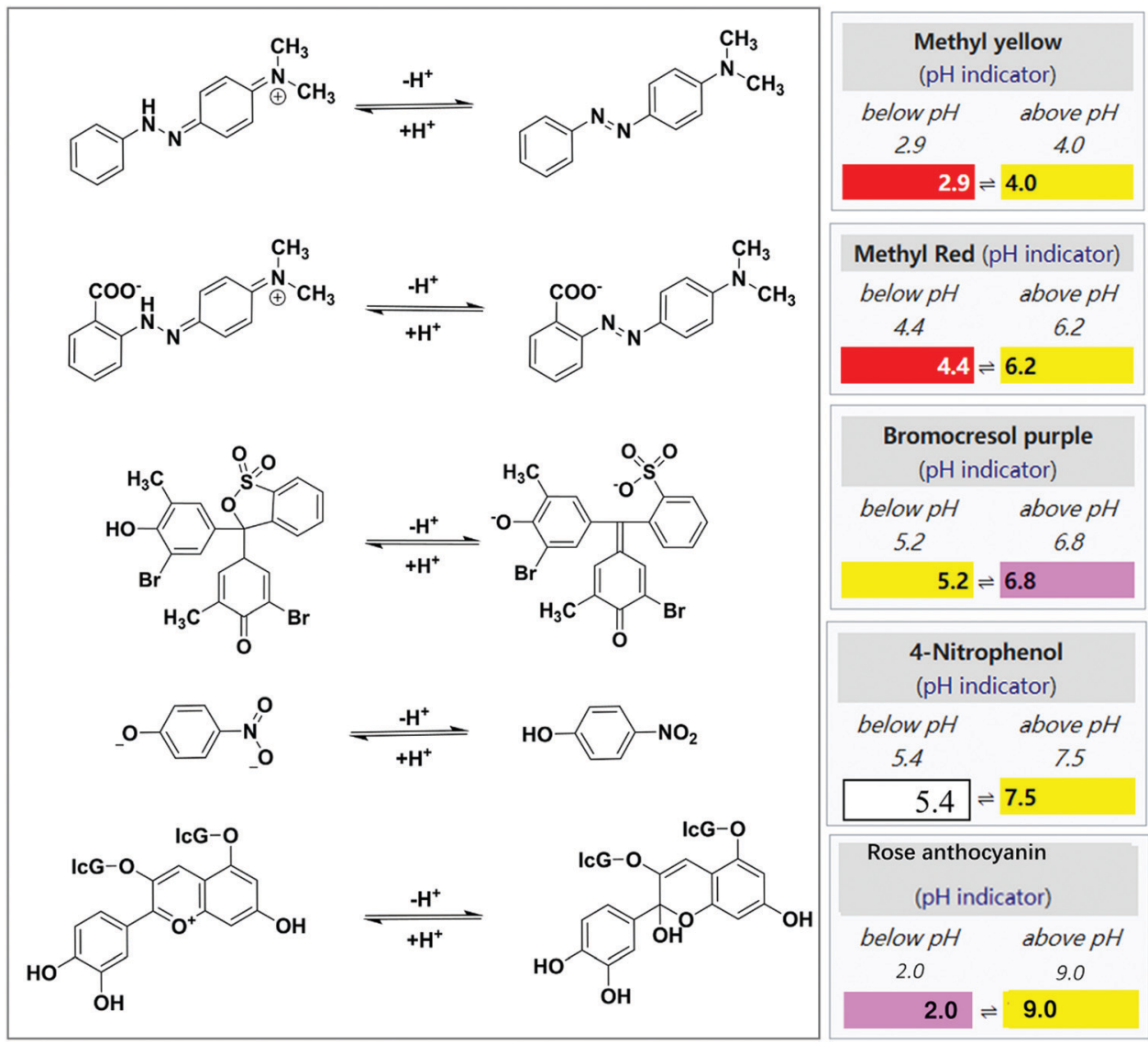

Fig. 1 Structure and color change of the $\mathrm{pH}$ indicators before and after protonation.

$2 \mathrm{HCHO}+\left(\mathrm{NH}_{2} \mathrm{OH}\right)_{2} \cdot \mathrm{HX} \rightarrow 2 \mathrm{H}_{2} \mathrm{C}=\mathrm{NOH}+\mathrm{HX}+2 \mathrm{H}_{2} \mathrm{O}$

The involvement of the classic organic aldimine condensation reaction and $\mathrm{pH}$-induced color change make this method simple. The most emphasis in this aspect is laid on the building of a stable and highly efficient sensing film. In 1994, Nakano et al. reported a disposable and porous cellulose paper impregnated with hydroxylamine sulfate $\left(\left(\mathrm{NH}_{2} \mathrm{OH}\right)_{2} \cdot \mathrm{H}_{2} \mathrm{SO}_{4}\right)$, MY, glycerin, and methanol, and applied it as a miniature colorimetric sensor to monitor FA with yellow to red color change. ${ }^{42}$ However, the LDL was only $100 \mathrm{ppb}$ at a sampling time of $30 \mathrm{~min}$, which cannot meet the detection standard of the WHO. In a follow-up study, they subsequently modified the disposable tape by adding silica gel as an adsorbent on its surface, optimizing the LDL down to $80 \mathrm{ppb} .{ }^{43}$ The silica gel was coated mainly to optimize the adsorption capability of the test paper toward gaseous FA.

In contrast to the above-mentioned low-cost cellulose tapes with rough morphological architectures, other platforms with controllable fiber morphology have been explored to improve the sensor response time and sensitivity. Owing to their exciting optical and structural properties (tunable morphology and size, large surface area, high porosity, interconnectivity, additional surface area, etc.), electro-spinning nanofiber/nets (NFN) membranes have been demonstrated as attractive building blocks for gas chemosensors. ${ }^{47-49}$ As schematized in Fig. 2a, Wang et $a l^{44}$ used nylon-6 NFN membranes as the platform for immobilizing the colorimetric sensing elements after their impregnation in the probe solution with the same components reported by Nakano et $a l .{ }^{43}$ When the MY-impregnated NFN membrane strips were exposed to FA vapor for $30 \mathrm{~min}$, the color changed from yellow to red (Fig. 2b). The sensor strips had an LDL of 50 ppb (Fig. 2b), and good reproducibility was obtained by repeated exposure to FA and treatment with the probe solution after 5 sensing cycles (Fig. 2c).

Similarly, Meng et al. ${ }^{45}$ developed an ormosil sensing coating having a nanoscale rill-like fold surface morphology, which was prepared by the sol-gel reaction of methyltriethoxysilance (MTES) and polydimethylsiloxane (PDMS). The sensor strip was prepared on a glass substrate through spin-coating with a mixture of hydroxylamine sulfate, rose anthocyanin, and ethanol. The sensor strip produced a yellowish-to-rosy color change when exposed to FA vapor. The LDL was $60 \mathrm{ppb}$ for $5 \mathrm{~min}$ sampling time. It should be mentioned that in view of the use of the stiff glass substrate, the pores of the ormosil coating could be easily blocked by the generated water molecules, which will slow the reaction speed.

In addition to hydroxylamine sulfate, there are many kinds of hydroxylamine salts (hydroxylamine hydrochloride $\left(\mathrm{NH}_{2} \mathrm{OH} \cdot \mathrm{HCl}\right)$, hydroxylammonium phosphate $\left(\mathrm{NH}_{2} \mathrm{OH}\right) \mathrm{HPO}$, etc., and their homogeneous compounds) that have been used 

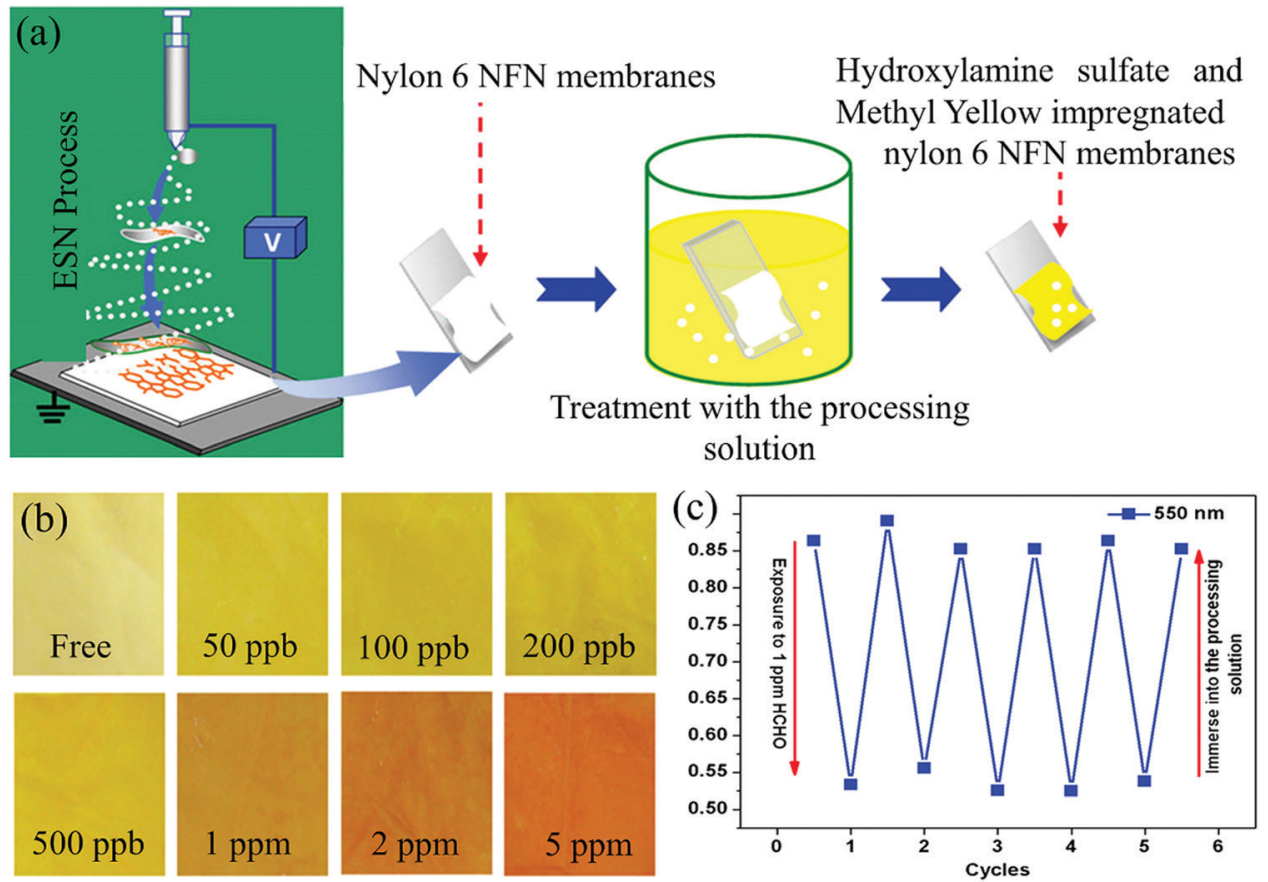

Fig. 2 (a) Schematic illustration of the fabrication, and (b) optical colorimetric response of the sensor strips upon exposure to increasing FA concentrations, and (c) repeated reflectance intensity $(550 \mathrm{~nm})$ changes of the sensor strips upon cyclic exposure to 1 ppm FA and treatment with the processing solution (reproduced with permission. ${ }^{44}$ Copyright: 2012, Elsevier).

for FA detection. The hydroxylamine salts containing hydrochloric acid $(\mathrm{HCl})$ are not very suitable for detection because the released $\mathrm{HCl}$ tends to be volatilized from the system. Such type of film reaction-based sensors could act as highly selective and sensitive strips for the detection of FA vapor with minor interference from other VOCs. However, as the reaction time increased, a dense monolayer of formaldoxime formed on the fiber surface, which limited the further contact of the FA molecules to hydroxylamine acid salts anchored on the substrate membrane. ${ }^{44}$ Nagashima et al. showed that the successful response to sub-ppm FA vapor usually depends on suitable hygroscopicity control with the help of humectants such as glycerin in the membrane and suitable relative humidity $(\mathrm{RH})$, accompanied by FA vapor in an external environment. ${ }^{42}$ Thus, commercially available aldehyde gas chemosensors based on this mechanism still have great challenges to give credible and sensitive sensing performances.

In addition to $\mathrm{pH}$-triggered colorimetric sensing strategy, the $\mathrm{pH}$ shift of the indicator dyes can be caused by aldimine condensation between the aldehyde and the polymercontaining amino groups in their molecular chains. Park et al. reported an efficient colorimetric sensor for gaseous FA based on the core-shell uniform poly(styrene-co-maleic anhydride) (PSMA)/ polyethyleneimine (PEI) nanoparticles impregnated with BCP, MR, or 4-nitrophenol. ${ }^{46}$ In this case, as shown in Fig. 3a, the shell of PEI acted as the sites for the formation of a Schiff base, and PSMA was selected as the core material and ion transporter because of the high reactivity between maleic anhydride and PEI. The colorimetric response was maximized at a PEI/PSMA ratio of 4 , probably owing to the high content of the amine groups.
Optimum FA gas detection was achieved at a $\mathrm{RH}$ of $30 \%$ using the MR colorimetric sensor, which exhibited a large color change (92\%) within 1 min (Fig. 3b). Moreover, the sensor achieved rapid naked-eye detection of low FA concentration of $500 \mathrm{ppb}$ (Fig. 3c).

2.1.2 Schiff reagents. Among the various Schiff base reactions, the Schiff test is quite an early and relatively general chemical test for the detection of various aldehydes. The Schiff reagent, also known as fuchsin sulfite reagent, which is a discolored product from the reaction of a magenta dye (fuchsin, pararosaniline, etc., as shown in Fig. 4a) with sodium bisulfite. The Schiff reagent could provide specific colorimetric response to aldehydes (e.g., violet color for FA and pink color for AA) and not to ketones. Although the mechanism of this sensing reaction is a bit complex, the aldimine-mediated method has been commonly acknowledged. ${ }^{50}$ Promisingly, this reaction also occurs in solid-state colorimetric films, offering advantages such as portability and ease of handling compared to conventional liquid-phase detection.

In 2008, Maruo et al. developed a highly sensitive sensor based on a Schiff's reagent (containing basic fuchsin, sodium sulfite, and $\mathrm{HCl}$ ), which could detect FA vapor through yellow or orange to red-violet color change with an LDL of $10 \mathrm{ppb}$ with 60 min exposure. ${ }^{51}$ In addition to the excellent selectivity to FA, the colorimetric film exhibited good repetitive response with a large concentration range from $20 \mathrm{ppm}$ to $10 \mathrm{ppb}$, benefiting from the porous glass sheets (with an average pore diameter of $4 \mathrm{~nm}$ ), which were used as the distinct substrate to impregnate both the Schiff's reagent and sulfuric or phosphoric acid. However, the possible adsorption of environmental organic compounds on the nanopores of the glass may weaken the 
(a)

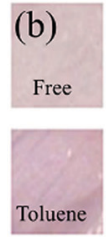

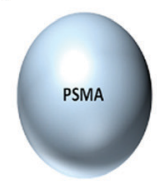
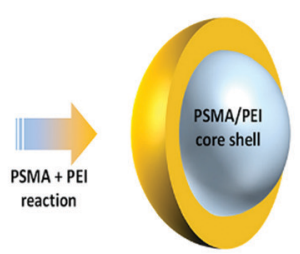

Step 2

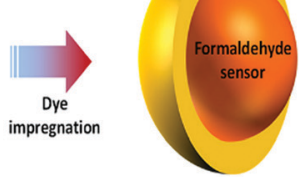

Step 3

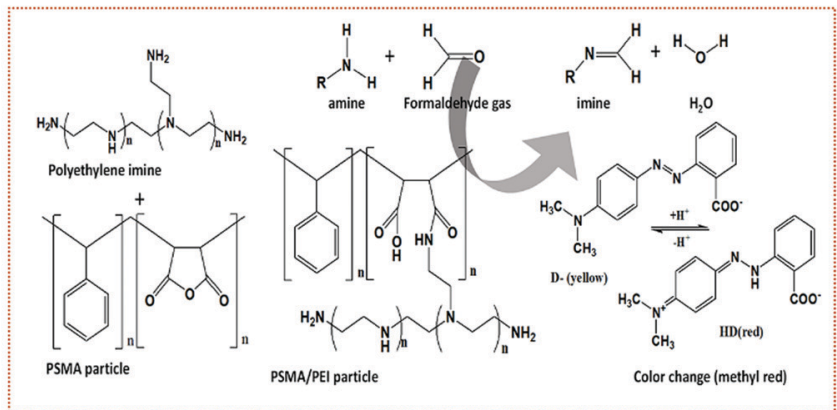

(b)

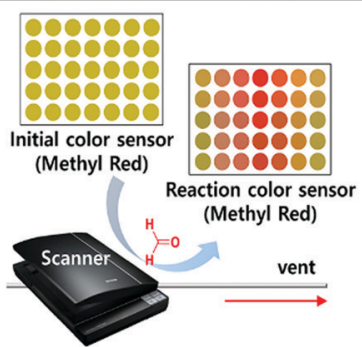

(c)

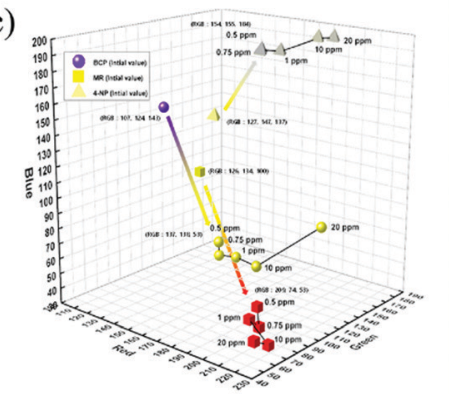

Fig. 3 (a) Schematic illustration of the preparation of a colorimetric sensor for gaseous FA based on PSMA/PEI core-shell nanoparticles doped with a pH indicator dye, and (b) the chromatic response of the sensor strips upon exposure to FA, and (c) the color changes observed for the colorimetric sensors based on $\mathrm{pH}$ indicators (BCP, MR, and 4-NP) when exposed to $0.5-20$ ppm FA (reproduced with permission. ${ }^{46}$ Copyright: 2020 , MDPI).
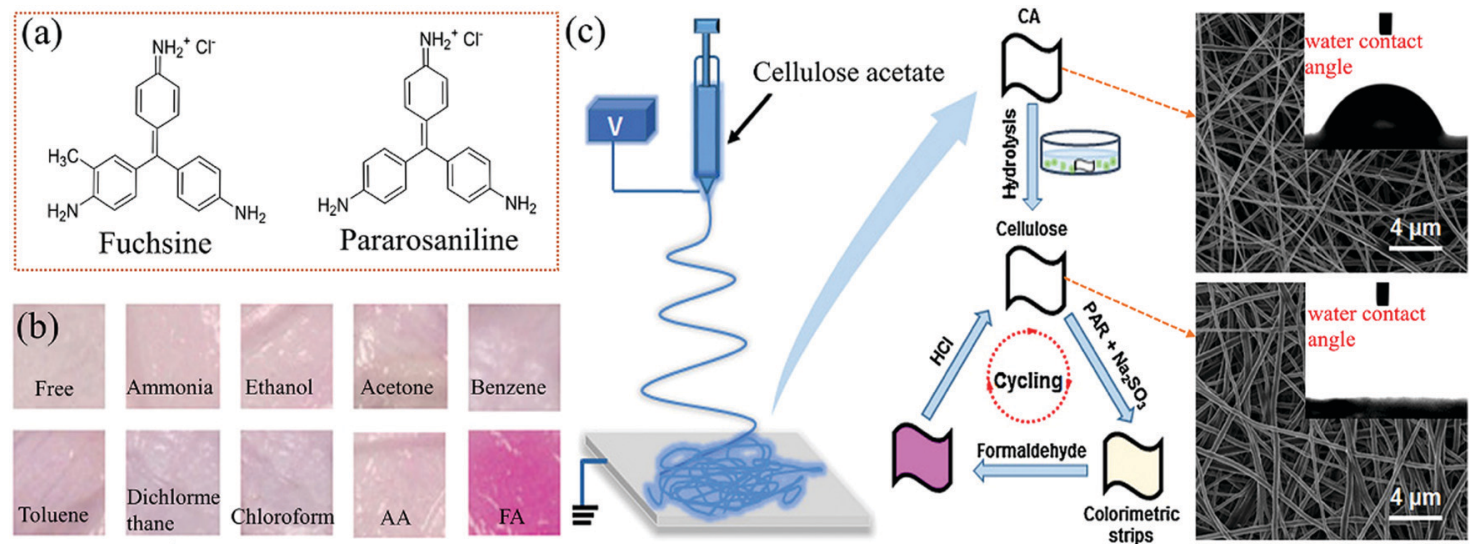

Fig. 4 (a) Chemical structures of fuchsin and pararosaniline. (b) Colorimetric response of the strips of the electrospun cellulose nanofibrous membranes immobilized with Schiff reagents upon exposure to $900 \mathrm{ppb} F A$ and various interfering vapors. (c) The fabrication procedure and morphology of these colorimetric strips (reproduced with permission. ${ }^{22}$ Copyright: 2014, Elsevier).

sensitivity in the ambient atmosphere. Later, in 2014, taking advantage of hydrophilic substrate modification, Ding et al. designed a reversible and stable colorimetric strip based on electrospun cellulose nanofibrous membrane, immobilizing the Schiff reagent (1:1 volumetric aqueous solution of pararosaniline and sodium sulfite) as the probe..$^{52}$ The preparation process of the sensing paper strips is shown in Fig. 4c. Based on the aldimine condensation reaction between FA and the Schiff reagent, the strip shows a distinct color transition from light pink to purple, even in the presence of trace-level FA and other interfering VOCs (Fig. 4b). The remarkably improved sensing performances of the cellulose nanofibrous membrane were attributed to the super-hydrophilicity and enlarged average diameter of the nanofibers of $410 \mathrm{~nm}$ size after the alkaline hydrolysis of the as-spun cellulose acetate membrane (possessing an average diameter of $c a .284 \mathrm{~nm}$ and a contact angle of $71^{\circ}$ ), as demonstrated in Fig. 4c. Benefiting from the apparently increased packing density, large specific surface area, and high porosity of the nanofibrous membranes, the strip exhibited improved naked-eye detection performances with an ultralow LDL of $40 \mathrm{ppb}$ and a short response time of 15 min under a constant temperature $\left(25{ }^{\circ} \mathrm{C}\right)$. Also, the strips showed good reproducibility with the help of $\mathrm{HCl}$; however, in order to realize fast and accurate detection, all the sensing tests required high RH (60\%). Obviously, further composition optimization of these nanofibrous membranes should be considered to meet their commercial use in a less humid environment. 
2.1.3 Amino-modified organic chromophores. Unlike the above $\mathrm{pH}$ indicator-incorporated chromogenic method, the development of intrinsic aldehyde-responsive chromophores as colorimetric sensors is more attractive. Indeed, recently, most commercially available FA vapor detectors usually employ cheap Sawicki's reagent solutions containing the probe compound 3-methyl-2-benzothiazolinonehydrazone hydrochloride hydrate (MBTH), which can first take part in aldimine condensation with aliphatic aldehydes, and subsequently give a colored reaction in an acid solution with the coexistence of an oxidizing agent such as ammonium iron(III) sulfate, after exposure to an atmospheric environment for tens of minutes (Fig. 5a and b). In this review, attention has been paid to organic chromophores without another color indicator.

\subsubsection{4-Amino-3-hydrazino-5-mercapto-1,2,4-triazole (AHMT).} 4-Amino-3-hydrazino-5-mercapto-1,2,4-triazole (AHMT) is an organic chromophore molecule that consists of a 1,2,4-triazole heterocycle ring with three functional groups-amino, thioamide, and hydrazide. Compared to traditional Schiff reagent and Sawicki's reagent, AHMT is one of the most commonly used colorimetric probe for monitoring the concentration of FA in the air because of its high AHMT sensitivity and special selectivity toward FA. ${ }^{53-57}$ Exceptionally, not only the coexistence of nitrogen dioxide, sulfur dioxide, and common VOCs but also vapors of ketones and other aldehydes cannot interfere in its quantitative determination toward FA at room temperature. The white transparent to violet-red color change comes from the aldimine reaction of AHMT after exposure to FA vapor in an alkaline solution, followed by oxidation using oxidizing agents such as potassium permanganate $\left(\mathrm{KMnO}_{4}\right)$ and potassium hydroxide (KOH) (Fig. 6a).

In recent years, many researchers have paid attention to the colorimetric sensing of FA vapor in real-time and the development of a portable analyzer instead of a large laboratory apparatus $^{54}$ to avoid naked-eye infeasibility or inaccuracy, ${ }^{55}$ or an automatically responding system. ${ }^{56}$ Kawamura et al. ${ }^{54}$ designed a hand-held sensor based on the disposable circular chips of the glass filter impregnated with AHMT and $\mathrm{KOH}$ solution. Under exposure to FA vapor at a rate of $400 \mathrm{~mL} \mathrm{~min}^{-1}$, the colorimetric gas sensor obtained an LDL of $40 \mathrm{ppb}$ with a shorter sampling time of $3 \mathrm{~min}$. More importantly, the applicability of various filter chips was carefully investigated. It was observed that the glass or silica is suitable compared to other materials such as cellulose, polyflon, polystyrene, cotton and sulfate paper which could possess abnormal color change of AHMT reagent even without exposure to gaseous FA. In addition, it was also observed that cellulose acetate showed a reactivity in the presence of FA. Such a difference among the substrates was attributed to the chemical inertness between AHMT reagent and glass filter chips. The identification of the color change via naked eyes is not reliable at a very low concentration, and the use of conventional absorbance spectrometer is cumbersome, higher in cost, not portable, and has complex instrumentation. The signal from sensing medium is recorded by measuring the reflected light with a photodiode after the irradiation of the sensing film by the light-emitting diode (LED) detector via a conventional light-path separated manner (Fig. 6b). Thus, the development of an alternative small, portable device setup for reliable color reading with much higher sensitivity, which can detect slight color changes, is desirable. A device smaller than a US dime was developed by Lin et $a .^{55}$ by vertically embedding a semitransparent finger-type organic photodetector (OPD) between a surface-mount device (SMD) LED and a fibrouspaper sensing film containing AHMT reagent (Fig. 6c). Such a compact sensing system can detect FA with concentration ranging from 40 to $1000 \mathrm{ppb}$ within $20 \mathrm{~min}$.

Furthermore, to realize an automatic measuring system for FA sensing, a smartphone-based microfluidic colorimetric sensor (Fig. 6d) was constructed by Chen et al. ${ }^{56}$ The primary parts of the microfluidic chip are illustrated in Fig. 6e. The microfluidic chip consists of two reagent reservoirs, one reaction reservoir, and a mixing column. A hydrophobic porous polytetrafluoroethylene (PTFE) membrane was kept on top of the reaction reservoir to prevent the fluid from flowing out while letting the gas molecules in. Using a microfluidic chip sensor, a smartphone-based FA determination system was developed, which makes the measuring process automated and simple.
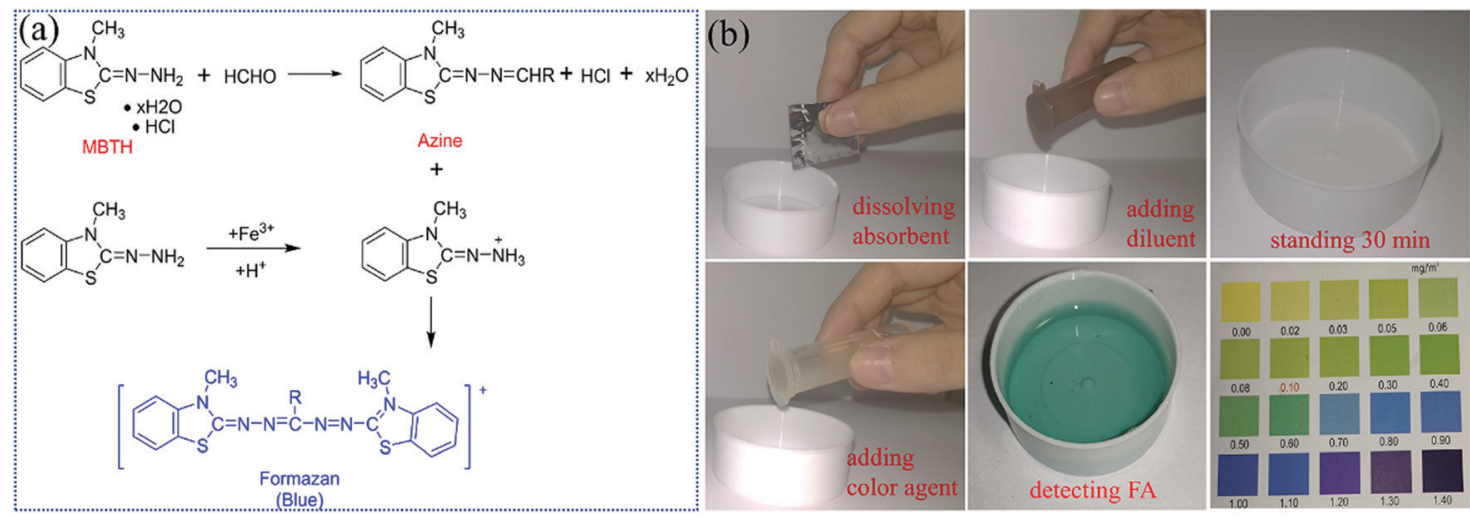

Fig. 5 (a) Schematic illustration of the FA detection reaction with MBTH. (b) Flow chart of the operation steps of FA detection based on a commercial MBTH method. 

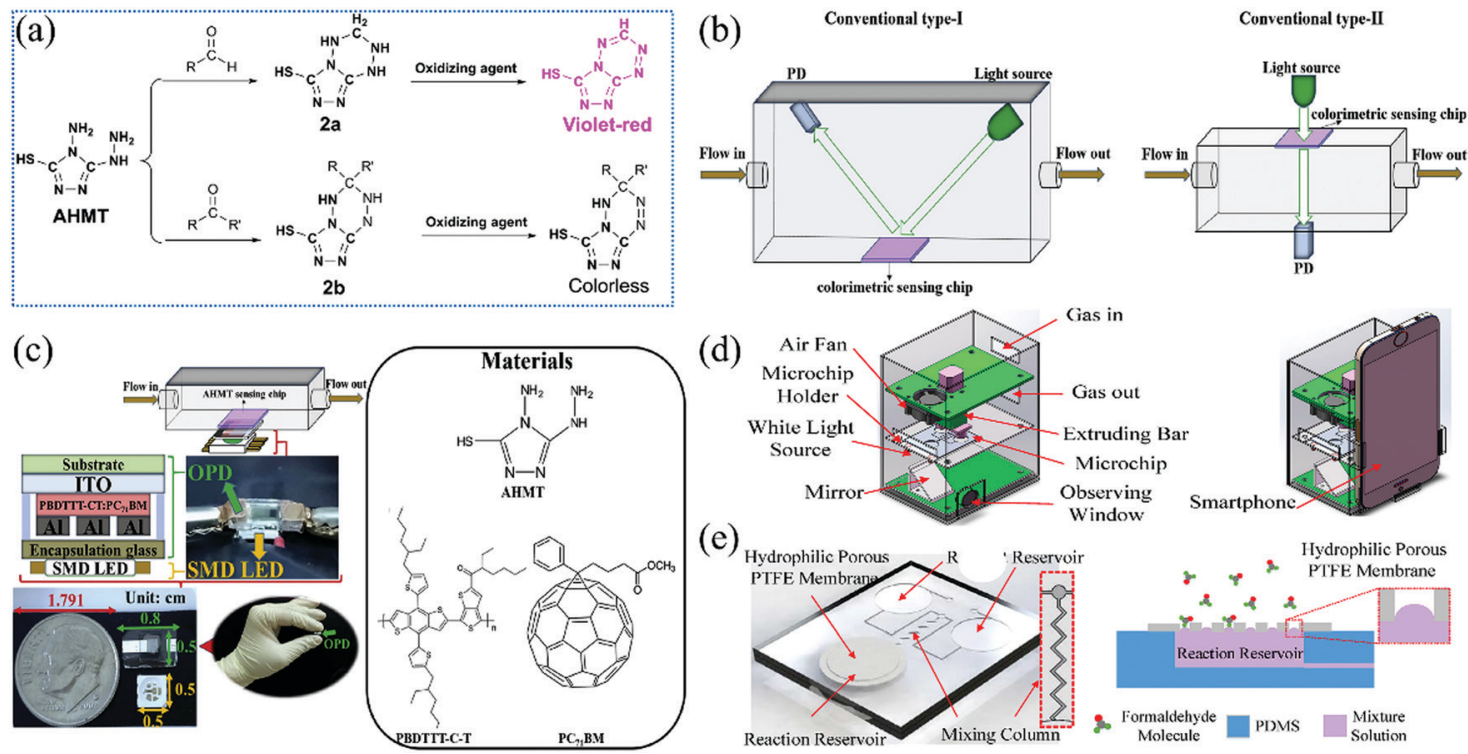

Fig. 6 (a) Chemical structure of colorimetric reagents (AHMT) and their transformation into lutidine derivatives after reaction with aldehyde and ketone. (b) Conventional type-I and Conventional type-II, and (c) the detailed structure of Conventional type-II (reproduced with permission. ${ }^{55}$ Copyright: 2019 , Elsevier). (d) FA determination system based on the smartphone, and (e) the primary parts of the microfluidic chip (reproduced with permission. ${ }^{56}$ Copyright: 2018, MDPI)

Although the operating temperature of about $20{ }^{\circ} \mathrm{C}$ and the controlled humidity within the range of $40-80 \%$ are very important for its LDL as low as $10 \mathrm{ppb}$ at a sampling time of $18 \mathrm{~min}$, such a smartphone-based FA determination system is quite suitable for indoor air monitoring.

2.1.3.2 2,4-Dinitrophenylhydrazine (DNPH). Unlike the special discrimination of AHMT toward FA, 2,4-dinitrophenylhydrazine (DNPH, Fig. 7a), named as Brady's or Borche's reagent, has been used as another well-known hydrazine dye. It can qualitatively respond to carbonyl groups associated with either aldehydes or ketones by the formation of brick red dinitrophenylhydrazone via the acid-catalyzed aldimine condensation reaction (Fig. 7a). A more yellow color change was observed toward saturated aliphatic analytes than unsaturated aliphatic or aromatic ones by this sensing mechanism. Researchers are actively involved in constructing the sensor arrays, which can play a crucial role in addressing a wide range of analytical challenges. ${ }^{58-60}$ By combining chemically reactive dyes with stoichiometric analysis, such colorimetric sensor arrays can be designed to distinguish various structurally similar analytes and analyte mixtures. Li et al. ${ }^{61}$ showed improved discriminatory power by a sensor array based on DNPH toward volatile aldehydes. This sensor array showed the sensitive (ranging from $40 \mathrm{ppb}$ to $10 \mathrm{ppm}$ ) and selective differentiation of nine kinds of C1-C7 aldehydes (Fig. 7b) with a short response time of about $4 \mathrm{~min}$ and with nearly no interference from sixteen other kinds of VOCs as lung cancer biomarker candidates. The theoretical LDL of FA gas can be down to $8.2 \mathrm{ppb}$ with a linear range from $10 \mathrm{ppb}$ to $150 \mathrm{ppm}$. The selection of dye and dye/DNPH ratio determines the sensitivity and specificity of the sensor array. It was reported that the selectivity of the sensor array depended on two characteristicsspecialized response of the dyes to different analytes and crossresponsive interaction between the analytes and the sensor (a)

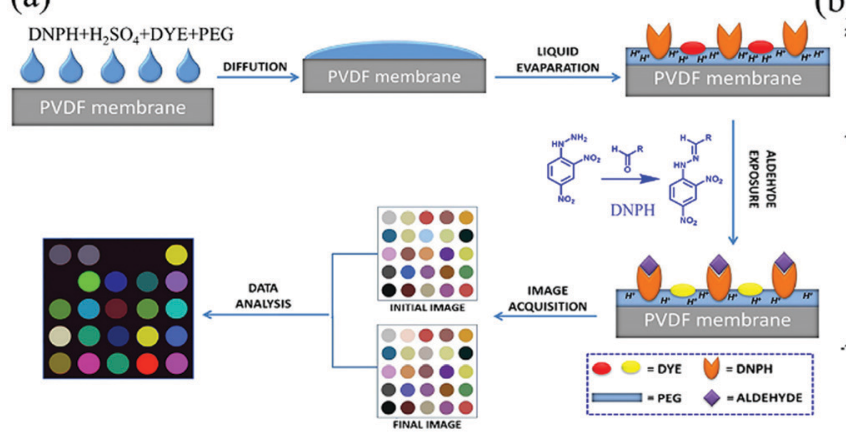

(b)

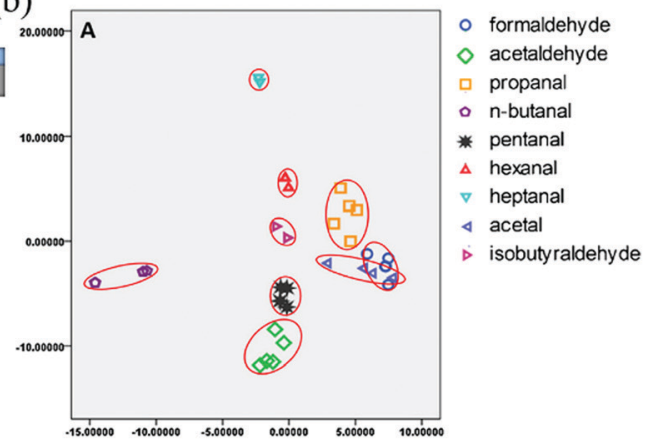

Fig. 7 (a) Schematic illustration of the procedure to develop the DNPH-based colorimetric sensor array, and (b) their linear discriminant analysis results of nine kinds of aldehydes with five parallel samples at the concentration of $40 \mathrm{ppb}$ (reproduced with permission. ${ }^{61}$ Copyright: 2014 , Elsevier). 
array. Herein, a stabilizer (polyethylene glycol) was necessary because it can protect dyes from an external environment and inhibit hydrophilic interference compounds such as water vapor, especially when this array sensor had to be stored in a $\mathrm{N}_{2}$-flushed container with a controllable $\mathrm{RH}$ of $50 \%$ and room temperature $\left(25^{\circ} \mathrm{C}\right)$ before being used. Other probe dyes support selective array response to structurally similar aldehydes. In addition, the experimental data of such a colorimetric sensor array highly depended on hierarchical cluster analysis and principal component analysis.

The use of high concentration strong acid $\mathrm{HClO}_{4}$ or $\mathrm{H}_{2} \mathrm{SO}_{4}$ to activate the dehydration reaction to hydrazones and the explosive hazard of DNPH, which is relatively sensitive to shock and friction, could weaken the future development of DNPHbased membrane gas sensors. In addition, as for colorimetric sensor arrays, the printing quality (e.g., the uniformity of color across an individual sensor spot or from one array to another) of the probing films has a significant influence on their sensitivity and accuracy. Sometimes, it is difficult to completely eliminate the variation of the array response to every gaseous analyte created by the non-reproducibility problem in printing. ${ }^{62}$

\subsection{Aldimine reaction-mediated biomimetic nanostructure tailoring}

In addition to chemical reaction or $\mathrm{pH}$-triggered sensing, other reactions also have been involved in the field of colorimetric gas sensors. In particular, liquid crystals or ordered nanostructures of biomimetic organic materials could undergo structural color changes by the alteration of their aggregation micro-/ nanostructures when exposed to external stimuli including volatile organic compounds (VOCs). ${ }^{63-66}$ With the assistance of the aldimine condensation reaction, amine-doped cholesteric liquid crystals (CLCs), ${ }^{67}$ amine-functionalized cellulose nanocrystals (CNCs) ${ }^{68}$ and self-assembled nanofibers of peptide amphiphiles (PAs) ${ }^{69}$ have been employed for the colorimetric sensing of gaseous aldehydes.

2.2.1 Amino-doped cholesteric liquid crystals (CLCs). Cholesteric liquid crystals (CLCs) exhibit colorimetric response not only toward the temperature due to their intrinsic liquid crystal feature but also toward chemical vapors due to the emergence of a vapor dissolution-induced concentration gradient in their bulk phase and subsequently the molecular orientation disruption effect of the intrinsic chiral structures of their molecules. ${ }^{70-72}$ The color changes are related to the molecular rotation, which triggers the wavelength change of the reflected light and can be reversed according to the vapor diffusing in or out. ${ }^{73}$ The CLC-based colorimetric sensors show relatively better response to aldehyde molecules with small size, especially FA. However, CLCs are more suitable for the selective detection of vapors, which have a large molecular size and can form hydrogen bonds with them, such as primary amines and alcohols rather than aldehydes. In view of this, the combination of aldimine condensation reaction with CLCs is a feasible method of detection.

By printing $2 \mathrm{wt} \%$ dodecylamine-doped CLCs films onto glass slides, Yang et al. ${ }^{67}$ successfully observed a color change from red to yellow-green upon the exposure of $300 \mathrm{ppm}$ pentyl aldehyde within $1 \mathrm{~min}$. No response was observed toward the vapors of water, ethanol, acetone, pentylamine, and pentyl alcohol. The estimated LDL for pentyl aldehyde, butyl aldehyde, and FA vapor are $2.77,4.99$, and $85.02 \mathrm{ppm}$, respectively, and the highest sensitivity is for pentyl aldehyde caused by the molecular size effect. Instead of the solvent phase, this research realized the amine-aldehyde reaction on the CLCs film, which was a melting mixture of cholesteryl benzoate, cholesteryl nonanoate, cholesteryl benzoate, and cholesteryl oleyl carbonate (Fig. 8a). Dodecylamine was used as a molecular receptor because the primary amine group of dodecylamine can react with an aldehyde group. First, they prepared a thin film of CLCs doped with $2 \mathrm{wt} \%$ dodecylamine, and half of the CLC thin film was covered with a clean glass slide. In this design, the CLC thin film under the glass slide does not come into contact with the aldehyde vapor. Therefore, this part did not produce any colorimetric responses after exposure to the aldehyde vapors, which makes it suitable to function as an internal visual control in this design. Remarkably, it was observed that even before exposure to any vapors, the doped CLCs showed different colors on the covered and uncovered parts. In contrast, undoped CLCs showed uniform color on both the parts. This result suggests that dodecylamine may adsorb at the cover slide and reduce the effective pitch of the CLCs. The authors proposed that the presence of dodecylamine lowers the surface energy and leads to a lower tilt angle of the CLC layer. To prevent the problem of having different colors on the covered and uncovered parts, firstly, a gas-permeable PDMS film $(\sim 100 \mu \mathrm{m})$ was placed on the entire surface of the CLCs doped with dodecylamine, and then half of the surface was covered with a glass slide, as shown in Fig. $8 \mathrm{~b}$ and c. In this design, the entire CLC thin film was in contact with PDMS only, and only the region not covered by the glass is permeable to gas. As a result, combining all the above designs, the sensor device showed good cycle reusability of the thin film for multiple exposures (Fig. 8d).

2.2.2 Amino-functionalized cellulose nanocrystals (CNCs). Another promising chromatic probing material, which works together with an external amino compound, is rod-like CNCs, which are derived from the hydrolysis process of native cellulose fiber sources caused by concentrated sulfuric acid treatment. ${ }^{65}$ It changes the optical properties under targeted stimuli such as humidity and VOCs via the controllable arrangement of their inherent liquid-crystalline structures. ${ }^{74}$ Since CNCs are much cheaper and,ore biocompatible compared to the synthesized CLCs, they are more suitable to develop as disposable and environment-friendly colorimetric film sensors for on-site gas detection. However, in contrast to the facile formation of their colored structures with chiral nematic phases in the liquid solutions due to the molecular self-assembly, the fabrication of their colored films on the solid substrates is more difficult. One critical reason is the presence of negatively charged surfaces due to the presence of abundant hydroxyl groups $\left(\mathrm{OH}^{-}\right)$and sulfate half ester motifs $\left(-\mathrm{C}-\mathrm{O}-\mathrm{SO}_{3}{ }^{-}\right)$, which inhibit the inter-particle interactions and the corresponding self-assembly process. ${ }^{68}$ Conversely, these groups are 
(a)

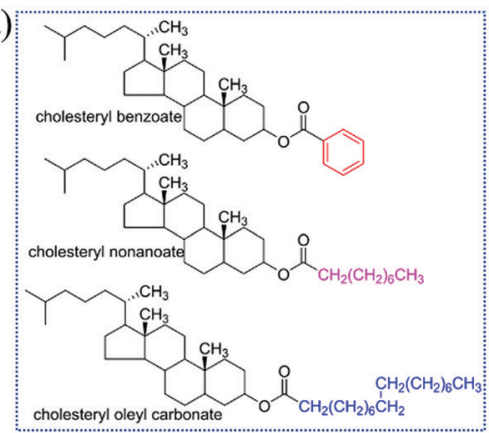

(c)
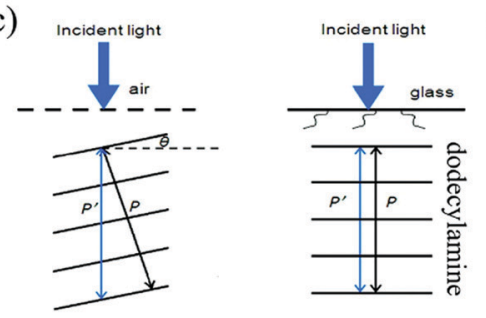

(b)

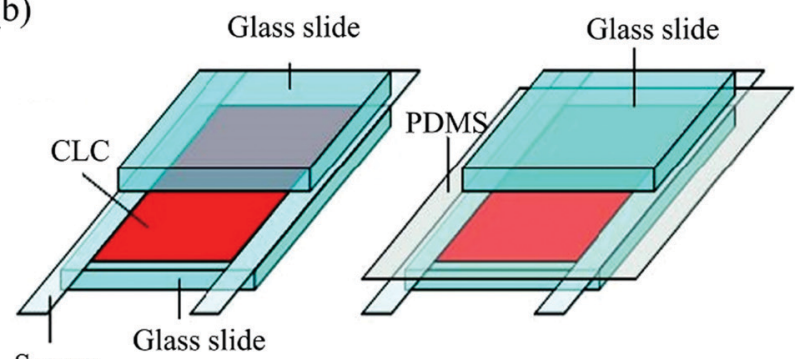

Spacer

Glass slide

(d)

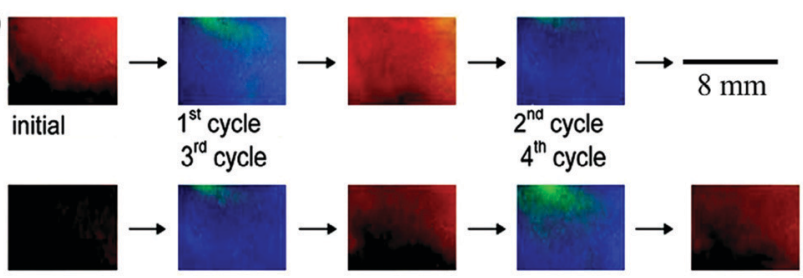

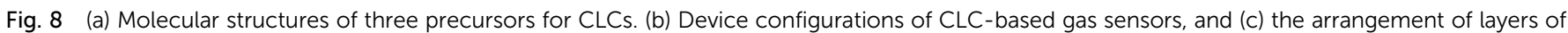

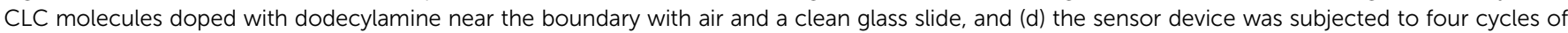

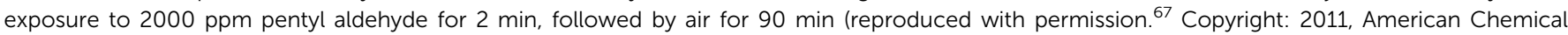
Society).

ideal for the further functionalization of CNCs because they are not responsive to aldehydes.

In 2018, by adjusting the assembly conditions of the CNC solution on stiff silicon wafer substrates with the assistance of ionic liquid during a dip-and-pull process, Song et al. ${ }^{68}$ fabricated a structure-stable CNCs films with a wider range of controllable colors, and subsequently developed a colorimetric gas sensor. The sensor film was chemically functionalized with amino groups to selectively detect aldehydes including FA and propyl aldehyde (Fig. 9a). In addition to a plastination function, the wise use of water-soluble ionic-liquid (1-butyl-3-methylimidazolium; BmimCl) molecules mainly aid in the close packing of the CNCs on the solid surface. This is because BmimCl could screen the repelling electrostatic charges between the CNCs owing to the chemical interactions between the $\mathrm{OH}^{-}$protons from cellulose and the $\mathrm{Cl}^{-}$anions from $\mathrm{BmimCl}$, as well as between the $\mathrm{Bmim}^{+}$cations and the ${ }_{-}^{-} \mathrm{C}-\mathrm{O}-\mathrm{SO}_{3}{ }^{-}$groups. It is clear that a suitable concentration ratio $(0.8 \mathrm{mg}$ BmimCl per $1 \mathrm{~mL}$ of CNC solution) should be severely considered. Such a robust assembled structure is the basis for realizing the clear color patterns of the CNCs films. An optimum pulling speed between 3 and $9 \mu \mathrm{m} / \mathrm{min}$ during the dip-and-pull fabrication process was also quite important for the control of the thickness (100-300 nm) of the thin CNC films and their varied colors (Fig. $9 \mathrm{~b}$ and c). The aldimine condensation reaction occurred between the amino groups and the aldehyde molecules and the films shows three color bands with both chemical and physical (e.g., swelling) changes. which results in a detectable change in the color patterns (Fig. 9d and e).
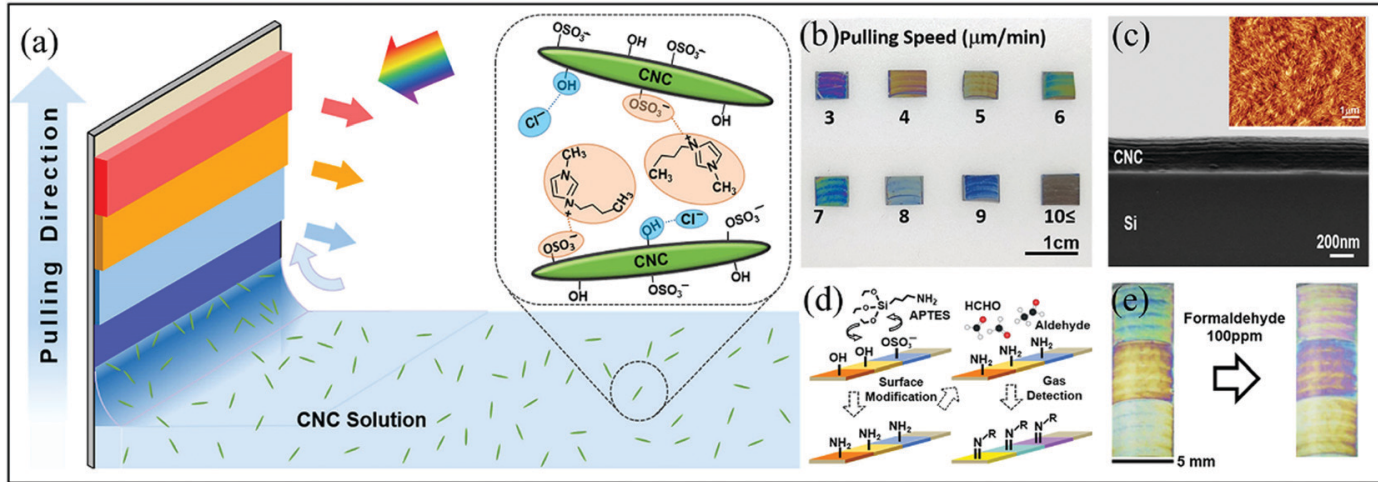

Fig. 9 (a) Schematic showing the fabrication of CNC-based color films on the solid substrates with the aid of ionic liquids, (b) the optical images of the assembled color bands at different pulling speeds, (c) the scanning electron microscopy (SEM) image of the cross section of the CNC-based colored film, (d) the schematic showing the surface modification and target gas binding of the CNC-based aldehyde gas sensors, and (e) the optical images of a CNC colorimetric sensor before (left) and after (right) exposure to 300 ppm FA vapor (Reproduced with permission. ${ }^{68}$ Copyright: 2018 , American Chemical Society). 
The film sensing capability can be recovered by exposing the film with water-bubbled $\mathrm{N}_{2}$ gas. It should be pointed out that the digital microscope camera was necessary to track the intensity of the emitting reflected light from multiple-color-band films in terms of the color change in the red/green/blue intensities $(\Delta \mathrm{RGB})$ for such lower ppm-level concentrations. Also, principal component analysis (PCA) should be employed to differentiate the aldehyde gases from other VOCs such as chloroform, isopropanol, and xylene.

2.2.3 Nanofibers assembled from peptide amphiphiles (PAs). Unlike the above-mentioned CLCs and CNCs films with an external amino group, colorimetric sensing toward gaseous aldehydes can be realized directly by nanofibril films of biomimetic peptide amphiphiles (PAs). ${ }^{75}$ On the one hand, in view of the structure of the hydrophobic tail (e.g., lipid alkyl chain) covalently conjugated to the hydrophilic peptide head, PAs molecules can self-assemble into various nanostructures with high aspect-ratio accompanied by color changes, arising from tunable intermolecular hydrogen bonding and electrostatic interactions under various conditions such as $\mathrm{pH}$, temperature, and ionic strength. ${ }^{76}$ On the other hand, bioactive peptides themselves have longer, continuous, unbranched peptide chain generated from the $-\mathrm{NH}_{2}$ and $-\mathrm{COOH}$ condensation of multiple amino acids. ${ }^{77}$ When basic amino acids (lysine or arginine) are used as the peptide source, the PAs could contain abundant amino groups on the surface of their nanostructured assemblies to support their interaction with the exposed aldehyde molecules. ${ }^{69}$

Recently, Gong et al. ${ }^{69}$ reported a colorimetric sensor fabricated from hierarchically structured Lysine-rich peptide amphiphiles (LRPAs) nanofibers with relatively uniform diameters of 7-9 $\mathrm{nm}$ for the selective detection of gaseous aldehydes (Fig. 10a and b). Similar to CNCs, the assembly structures and colors of the LRPAs films can also be tailored by controlling the dipping-pulling process conditions (especially, extraction speeds) on aluminum-coated silicon substrates from the peptide solution with a certain $\mathrm{pH}$ and concentration. When exposed to a range of volatile aldehydes, including FA, AA, propanal, and hexanal, the color band patterns exhibited detectable $\Delta$ RGB color changes within $1 \mathrm{~min}$ at concentrations as low as $10 \mathrm{ppm}$, largely due to the swelling/de-swelling process of the probe structures upon the aldimine reactions. PCA was also implemented to quantitatively identify their distinct and reproducible color changes to differentiate themselves from each other and from non-aldehyde VOCs (diethyl ether, hexane, acetone, methanol, etc.) at concentrations of 10-300 ppm (Fig. 10c and d). More promisingly, in contrast to liquid crystals, the pristine LRPAs film showed slight color change even at elevated temperature $\left(70{ }^{\circ} \mathrm{C}\right)$ before exposure to VOCs, which may provide a chance to shorten the response time by enhanced gas vaporization and diffusion via the heater element within an integrated analyzer.

\subsection{Sensors based on the Hantzsch reaction}

The Hantzsch reaction is one of the old and famous multicompetent reactions toward pyridine synthesis at room conditions, using a mixture of an aldehyde, two equivalents of a $\beta$-ketoester or $\beta$-diketone, and a nitrogen donor such as amino or ammonium (Fig. 11a). ${ }^{78}$ Thus, this one-pot three-component reaction has been used to quantitatively determine trace aldehydes under mild conditions without any intermediate materials. In most cases, the colorimetric gas sensor was constructed by impregnating the colorless solutions of $\beta$-diketone and ammonium ions within porous substrates such as optically transparent glass and sol-gels. Upon exposure to FA vapor, a colored pyridine product would be synthesized, usually yellow lutidine or its derivative products. Moreover, the LDL was slightly high for nontransparent substrates because their color change is measured by the reflected absorption.

Maruo et ll $^{79}$ presented the Hantzsch method to detect FA vapor grounded on a porous glass chip (with $1 \mathrm{~mm}$ thick and an average pore diameter of $4 \mathrm{~nm}$ ) impregnated with a solution of acetic acid, ammonium acetate, and one of three kinds of

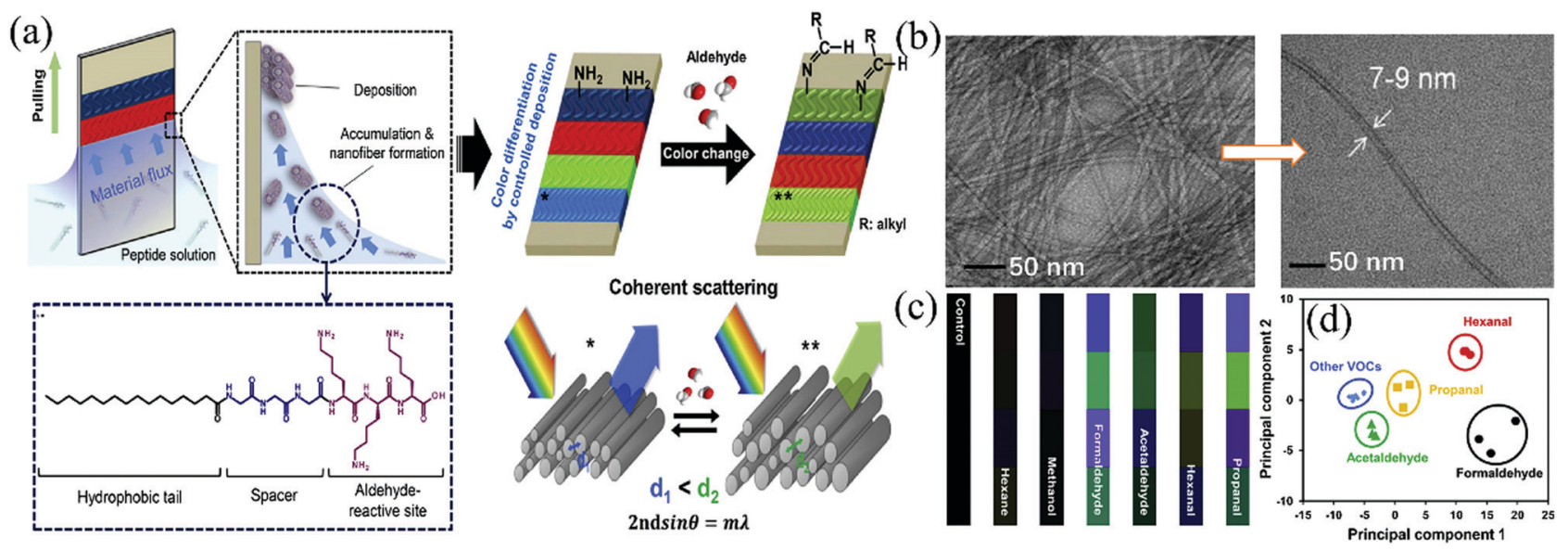

Fig. 10 (a) Schematic of the fabrication and operation mechanism of lysine-rich peptide-based color sensors for gaseous aldehyde detection, (b) the TEM images of the LRPA nanofibers, (c) differential color change of the LRPA sensor in response to various aldehyde and non-aldehyde gases at a concentration of $10 \mathrm{ppm}$, and (d) 2-dimensional PCA plot for the aldehydes and various non-aldehyde organic solvents at 10 ppm (Reproduced with permission. ${ }^{69}$ Copyright: 2019, Elsevier). 

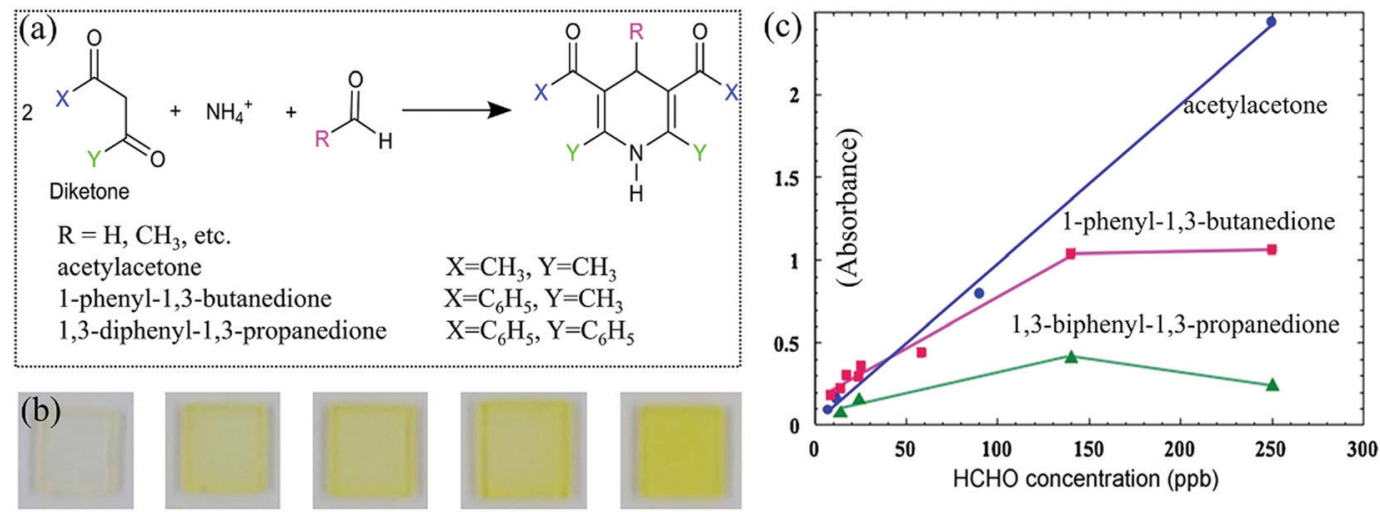

Fig. 11 (a) The reaction between FA and diketone. (b) Photographs of the acetylacetone-impregnated sensor element before and after exposure to FA: before exposure, 14 ppb, 24 ppb, 62 ppb, 110 ppb (from left to right). (c) Relationship between the exposure concentration and the peak absorbance of the three sensors (exposure time $=24$ h) (reproduced with permission. ${ }^{79}$ Copyright: 2008 , Elsevier).

$\beta$-diketones, which was subsequently dried at $20{ }^{\circ} \mathrm{C}$. When exposed to FA with increased concentrations, the sensor turned from colorless to deep yellow (Fig. 11b), corresponding to a shift in the specific absorbance from wavelengths below $340 \mathrm{~nm}$ to $407 \mathrm{~nm}$ for acetylacetone, $370 \mathrm{~nm}$ to $414 \mathrm{~nm}$ for 1-phenyl-1,3-butanedione, and $400 \mathrm{~nm}$ to $424 \mathrm{~nm}$ for 1,3diphenyl-1,3-propanedione. Under the same concentration of FA, the intensity of absorbance changes were in the order acetylacetone $>$ 1-phenyl-1,3-butanedione $>$ 1,3-diphenyl-1,3propanedione (Fig. 11c). The wavelength of the peak was $7 \mathrm{~nm}$ longer than that of acetylacetone because of the substituent effect of one benzene ring that is included in 1-phenyl-1,3butanedione. It was reported that both the formation and decomposition reactions of the lutidine derivative (yellow dye) occur on the acetylacetone element at $20^{\circ} \mathrm{C}$. On the other hand, only the formation reaction occurs on the 1-phenyl-1,3butanedione element at $20{ }^{\circ} \mathrm{C}$. The acetylacetone sensor element was suitable for short-term measurements (e.g., less than $8 \mathrm{~h}$ ), and the 1-phenyl-1,3-butanedione sensor element was suitable for long-term measurements (e.g., more than $8 \mathrm{~h}$ ). To improve the poor response time of about $24 \mathrm{~h}$, they ${ }^{80}$ further developed a 1-phenyl-1,3-butanedione-based portable sensing device to achieve hourly response to indoor FA with an LDL of $3.5 \mathrm{ppb}$ and an estimated reaction rate of $0.049 \mathrm{~min}^{-1}$. Meanwhile, the sensitivity was stable within the range of $15-35{ }^{\circ} \mathrm{C}$ and $30-70 \%$ humidity. Such a sensor also showed a negligible color-fading interference of $\mathrm{SO}_{2}$, which is not negligible for $\mathrm{NO}_{2}, \mathrm{O}_{3}$ in residential indoor air or indoor light. Moreover, it was interference-free from 2000 ppm common VOCs such as benzenes, alcohols, ketones, acetic acid, ethyl acetate as well as $200 \mathrm{ppm}$ of AA, propanal, nonylaldehyde, benzaldehyde, and styrene using the same experimental method.

Besides porous glass substrates, Kanatharana et al. ${ }^{81}$ achieved an LDL of $0.03 \mathrm{ppm}$ for FA based on an acetylacetone-based sensor with a soft sol-gel matrix in a disposable UV cuvette. The Hantzsch reaction can be completed within $2 \mathrm{~h}$, which is about three times faster than that between FA and methyl acetoacetate under the same conditions. Also, in view of the effect of the ester group, the maximum adsorption wavelength for the product from acetylacetone is $410 \mathrm{~nm}$ compared to $376 \mathrm{~nm}$ of that from methyl acetoacetate, leading to a stronger yellow coloration for reliable detection. This sol-gel sensor could discriminate FA from common interfering carbonyl compounds, e.g., aldehydes (AA, benzaldehyde, etc.) and ketones (acetone, butanone, etc.) due to its high selectivity. This sensor showed 15.5 times faster response toward FA compared to AA. Although the Hantzsch reactionbased method is simple and selective, the sensitivity is not satisfactory for FA detection (sampling time $>1 \mathrm{~h}$ ).

\subsection{Sensors based on the Sawicki reaction}

By integrating the amine source into the skeleton structure of dienone molecules, researchers developed the Sawicki reaction, which is a derived Hantzsch reaction that transforms colorless enaminones to yellow lutidine derivatives. ${ }^{82}$ The colorimetric chemosensor was developed by various research groups by entrapping enaminones (especially 4-amino-3-penten-2-one (Fluoral-p) and its phenyl derivatives, 4-amino-4-phenylbut-3en-2-one (KD-XA01) and 3-amino-1,3-diphenylprop-2-en-1-one (KD-XA02)) into the micro-/nanoporous thin film substrates. The chemosensor exhibited quantitative, selective, and sensitive response to FA vapor due to the formation of the detectable product, 3,5-diacetyl-1,4-dihydrolutidine (DDL) or its substituted derivatives. ${ }^{83-85}$ No interference was observed from common indoor gaseous pollutants such as chloromethanes, benzenes, ammonia, carbon oxides, nitrogen oxides, and other carbonyl compounds (AA, benzaldehyde, ketones, alcohols, etc.). The temperature variations also did not influence the kinetics in the temperature range of $15-35{ }^{\circ} \mathrm{C}$. It is worthwhile to note that AA vapor also can undergo the first stage reaction with Fluoral-p via water elimination in the second stage, leading to the formation of different end products (Mannich base) without color change. However, water (approximately $12000 \mathrm{ppm}$ at $50 \% \mathrm{RH}$ ) is the main interferent, which can cause the irreversible hydrolysis of Fluoral-p to form acetylacetone and ammonia. Thus, the influence from high RH has to be taken care of during the fabrication of the sensing films, considering their practical use. Another detrimental effect of 
high humidity was also demonstrated by Tran thi and Hue et al. ${ }^{83,84}$ where the filling of the hydrophilic nanopores with a diameter ranging from 0.8 to $2.0 \mathrm{~nm}$ by the water molecules inhibited the diffusion of the FA vapor inside the probe films. These nanoporous films with high specific surface areas were obtained via the room-temperature sol-gel processes from alkoxysilane precursors $\left(\mathrm{Si}(\mathrm{OR})_{4}\right)$ and/or modified alkoxysilane precursors $\left(\mathrm{R}^{\prime} \mathrm{xSi}(\mathrm{OR})_{4-2 x}\right)$, which have often been used to encapsulate the dye molecules for chemosensor applications (Fig. 12a). The ratio of silicane precursors and the gelation conditions are critical to tailor the sol-gel microstructure and the polarities are also important to trap the desired analyte molecules inside the pore. In one way, monolithic matrices of hybrid inorganic sol-gel polymers were prepared by pouring the aqueous solution of tetramethoxysilane (TMOS) and (3-aminopropyl)triethoxysilane (APTES) with Fluoral-p solution in methanol in specific molds, followed by heating. In another way, with the help of the photolithography technique to control the hydrophobic and hydrophilic zones of microscope glass slides. The hybrid organic-inorganic polymer films with various shapes and thicknesses were spin-coated onto their surfaces from aqueous-ethanol sol-gel solutions of silicon precursor TMOS and methyltrimethoxysilane (MeTMOS) with Fluoral-p ${ }^{84}$ (Fig. 12b). The use of modified alkoxysilane precursors APTES or MeTMOS with a faster hydrolysis rate than TMOS not only provides the silica-based hybrid films containing $\mathrm{Si}-\mathrm{O}-\mathrm{Si}$ and $\mathrm{Si}-\mathrm{C}$ bonds but also decreases the local polarity of the pores to eliminate $\mathrm{RH}$ interference by reducing the retention of water vapor. ${ }^{86}$ Nevertheless, a response time of $2 \mathrm{~h}$ can be achieved toward $>60 \mathrm{ppb}$ FA at $50 \% \mathrm{RH}$ by this design.
To avoid the problem of pore blocking by the water molecules, Ding et al. ${ }^{87}$ utilized 4-amino-3-penten-2-one (Fluoral-p)functionalized electrospun polyacrylonitrile (PAN) (PAN/ Fluoral-p) nanofibrous membranes for the naked-eye colorimetric assay of FA. The PAN/Fluoral-p colorimetric sensor strips were fabricated by dip-coating a piece of PAN fibrous membranes into 1 wt $\%$ Fluoral-p solution. The Fluoral-p solution was prepared by dissolving $0.1 \mathrm{~g}$ of Fluoral-p in a mixed solution consisting of methanol, glycerin, and phosphate buffer solution (PBS) (Fig. 12c). Due to the large specific areas, high porosity, and hydrophilicity toward FA vapor, the formed PAN/ Fluoral-p sensor strips possessed much more sensitive response with a lower naked-eye LDL of $40 \mathrm{ppb}$ than that of filter paperbased colorimetric sensor strips prepared by the same procedure including silicon films based on the sol-gel method. Since $\mathrm{H}^{+}$ can act as the activator to accelerate the Mannich and Sawicki reaction (Fig. 12d), the pH values of PBS should be carefully adjusted to reach such a high sensitivity. In particular, a low $\mathrm{pH}$ range $(<2.5)$ is optimized.

To reduce the hydrolysis of Fluoral-p, as shown in Fig. 13a, its aryl derivatives 4-amino-4-phenylbut-3-en-2-one (KD-XA01) and 3-amino-1,3-diphenylprop-2-en-1-one (KD-XA02) with good stability have been used for the Sawicki reaction-based colorimetric FA sensor. ${ }^{85}$ Suzuki et al. inserted a porous cellulose filter paper into the solution containing the methanolic solution of KD-XA01 and PBS ( $\mathrm{pH} 2.5)$, and silica gel as an adsorbent, and then dried under reduced pressure and nitrogen stream protection for $60 \mathrm{~min}$. The obtained film sensor had an LDL of $5 \mathrm{ppb}$ in a sampling time of $15 \mathrm{~min}$. Although it has a much longer lifetime (6 months) at high temperature and humidity, they can still have an influence on its sensitivity

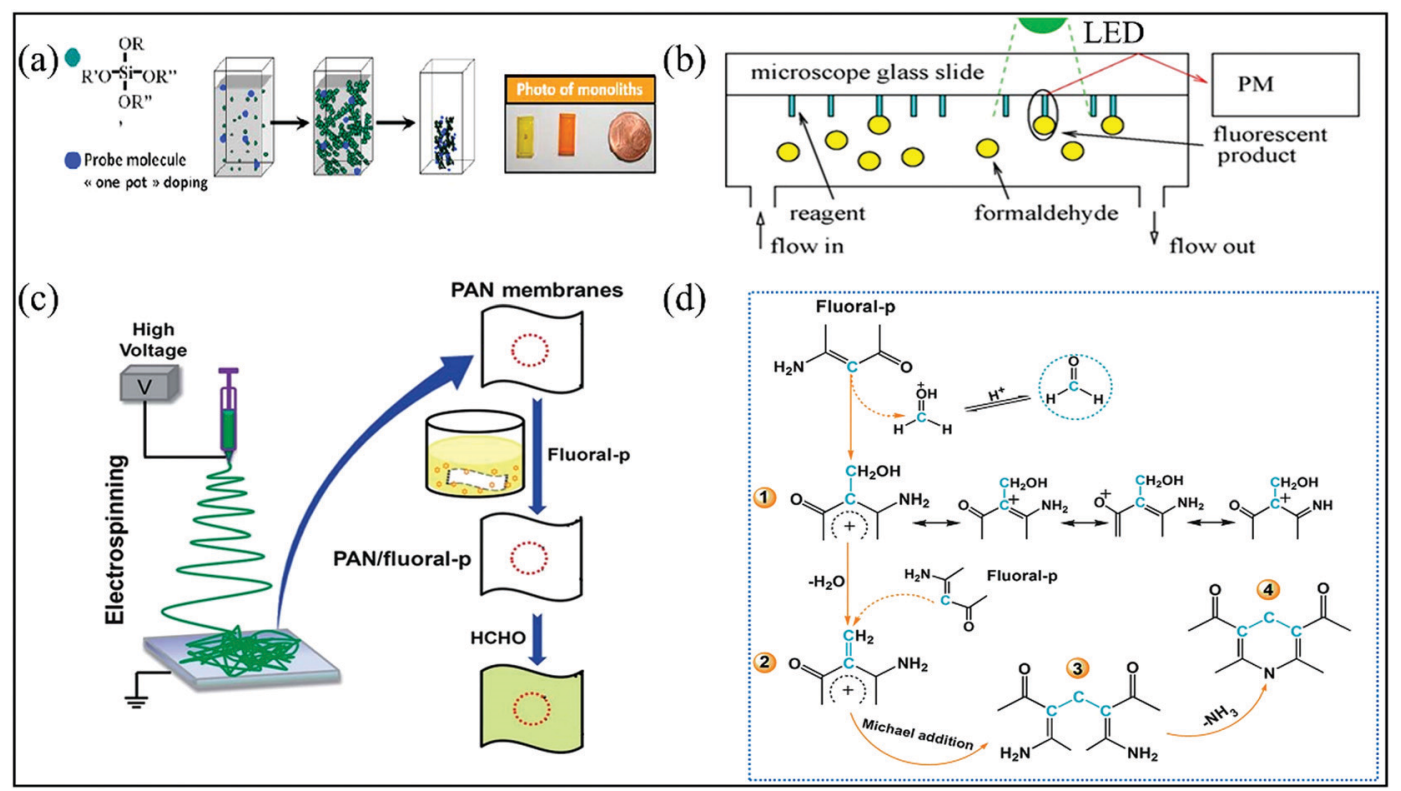

Fig. 12 (a) Schematic representation of the synthesis of the nanoporous monoliths doped with Fluoral-p (reproduced with permission. ${ }^{83}$ Copyright: 2010, Elsevier). (b) Detection principle of the apparatus (reproduced with permission. ${ }^{84}$ Copyright: 2012, Elsevier). (c) The procedure for the fabrication of PAN/Fluoral-p colorimetric sensor strips, and (d) the schematic representation of the proposed sensing mechanism (reproduced with permission. ${ }^{87}$ Copyright: 2013, Royal Society of Chemistry). 


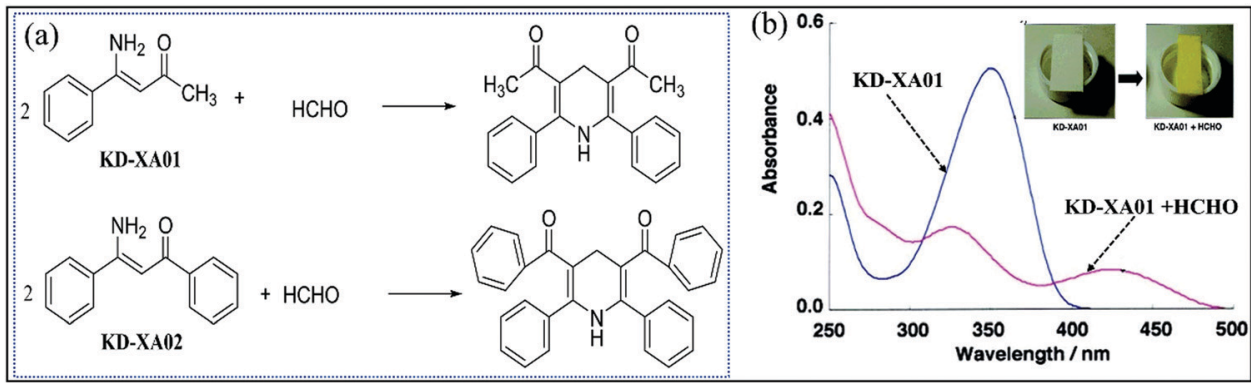

Fig. 13 (a) Chemical structures of the colorimetric reagents (KD-XA01 and KD-XA02) and their transformation into lutidine derivatives after reaction with $\mathrm{HCHO}$. (b) Absorption spectra of KD-XA01 before and after reaction with $300 \mathrm{ppb}$ FA at room temperature (reproduced with permission. ${ }^{85}$ Copyright: 2003, American Chemical Society).

under low concentration conditions $(<10 \mathrm{ppb})$. Promisingly, its response toward FA $(>10 \mathrm{ppb})$ was not affected by the $\mathrm{RH}$ in the range from $30 \%$ to $70 \%$ at $25{ }^{\circ} \mathrm{C}$. KD-XA01 itself and its yellow product with FA showed a red-shifted absorption peak of reflected light at $350 \mathrm{~nm}$ and $425 \mathrm{~nm}$, respectively (Fig. 13b), compared to $325 \mathrm{~nm}$ and $420 \mathrm{~nm}$ for that of KD-XA02. Thereby, the KDXA01-based sensor has a faster response rate than that based on KD-XA02.

\subsection{Tollens' reaction-mediated LSPR}

The Tollens' reaction is another well-known identification reaction to distinguish aldehydes from other interference analytes including ketones (except alpha-hydroxy ketones), alcohols, anilines, phenols, and other common organic chemicals (alkanes, furans, acetates, etc.). As shown in eqn (2) and (3), a silver mirror forms after dropping Tollens' reagent into an analyte solution, which is an aqueous alkalescent $\left[\mathrm{Ag}\left(\mathrm{NH}_{3}\right)_{2}\right]^{+}$ solution consisting of silver nitrate, an ammonium salt, and sodium hydroxide $(\mathrm{NaOH})$.

$$
\mathrm{HCHO}+4\left[\mathrm{Ag}\left(\mathrm{NH}_{3}\right)_{2}\right] \mathrm{OH}=\left(\mathrm{NH}_{4}\right)_{2} \mathrm{CO}_{3}+4 \mathrm{Ag} \downarrow+6 \mathrm{NH}_{3}+2 \mathrm{H}_{2} \mathrm{O}
$$

$2\left[\mathrm{Ag}\left(\mathrm{NH}_{3}\right)_{2}\right]^{+}+\mathrm{RCHO}+\mathrm{H}_{2} \mathrm{O} \rightarrow 2 \mathrm{Ag}(\mathrm{s})+4 \mathrm{NH}_{3}+\mathrm{RCOOH}+2 \mathrm{H}^{+}$

In recent decades, LSPR sensing has attracted a great deal of interest as a rapid and label-free technique for biological and chemical sensor applications. ${ }^{88}$ One of the most essential characteristics of metal NPs such as Au and Ag is their LSPR, which refers to the collective oscillation of the conducting electrons of the metal NPs when their frequency matches that of the incident electromagnetic radiation. Strong absorption or increased scattering intensity of the radiation occurs at certain wavelengths for the metal NPs as a result of this phenomenon. The inherent surface plasmon optical absorption feature in the visible light region of Ag NPs and Au NPs can be well adjusted by the chemical interactions (electrostatic, hydrogen bond, chemical reaction, etc.) between them and the target analyte molecules, leading to color variation. In general, a change of color from yellow to brown for Ag NPs and from red to blue for $\mathrm{Au}$ NPs corresponding to their transformation from well-spaced to aggregated state was observed ${ }^{89}$ Although Au NPs are more chemically and structurally stable, Ag NPs are a more popular choice in LSPR sensing due to their cheaper, wide range of plasmon adsorption wavelengths across $300-1200 \mathrm{~nm}$, stronger LSPR (i.e., highly sensitive to light excitation), and sharper absorbance peak. As a consequence, a variety of Tollens' reaction-mediated LSPR sensors have been developed for the visual detection of aldehydes in the solution phase, using the indirectly quantitative detection of LSPR adsorption intensity instead of the color change signal. ${ }^{90-94}$ In general, a polymer matrix stained with Tollens' reagent was employed as the wet probe, which was immersed into a solution mixture containing a strong alkali, a molecular mediator (usually a surfactant as the stabilizer), and the aldehyde analyte. The polymer matrix should be resistant to acid and alkali corrosion. Then, a UV-Vis spectrophotometer was used to record the LSPR absorption of $\mathrm{Ag}$ NPs embedded in the polymer matrix. These approaches exhibit excellent selectivity to aldehydes driven by the Tollens' reaction, and high sensitivities due to the strong LSPR effect of the generated Ag NPs in sequence. Although all aldehydes have a mild reducing power, FA has the fastest response and could still be differentiated from other aldehydes such as AA or benzaldehyde, even when using 100 times low concentration. This is probably due to its simplest molecular structure with virtually two aldehyde groups for the rapid Tollens' reaction, and relatively stronger diffusivity and reductive capability. The LSPR of the Ag, Au NPs and their sensing behavior is mainly related to the NP size, shape, composition, interparticle distance, and dielectric constant (refractive index) of the surrounding medium.

Huang et al..$^{90}$ reported that uniform Ag nanocubes with a mean edge length of $200 \mathrm{~nm}$ can be formed on the Nafion film by the reaction between FA and Tollens' reagent in the presence of adenosine-5'-triphosphate (ATP) disodium salt hydrate as a molecular mediator. A plasmon absorption band was recorded at $420 \mathrm{~nm}$ corresponding to a color change from colorless to yellow (Fig. 14a) as well as a plasmon light scattering signal was recorded at $560 \mathrm{~nm}$ by a fluorescence spectrophotometer with strongly scattered green light under white light excitation. ATP and its concentrations were necessary for morphology formation and to adjust the size-controllable and monodisperse $\mathrm{Ag}$ nanocubes on the Nafion film. The operation temperature $\left(25-28^{\circ} \mathrm{C}\right)$ was another important factor to support 

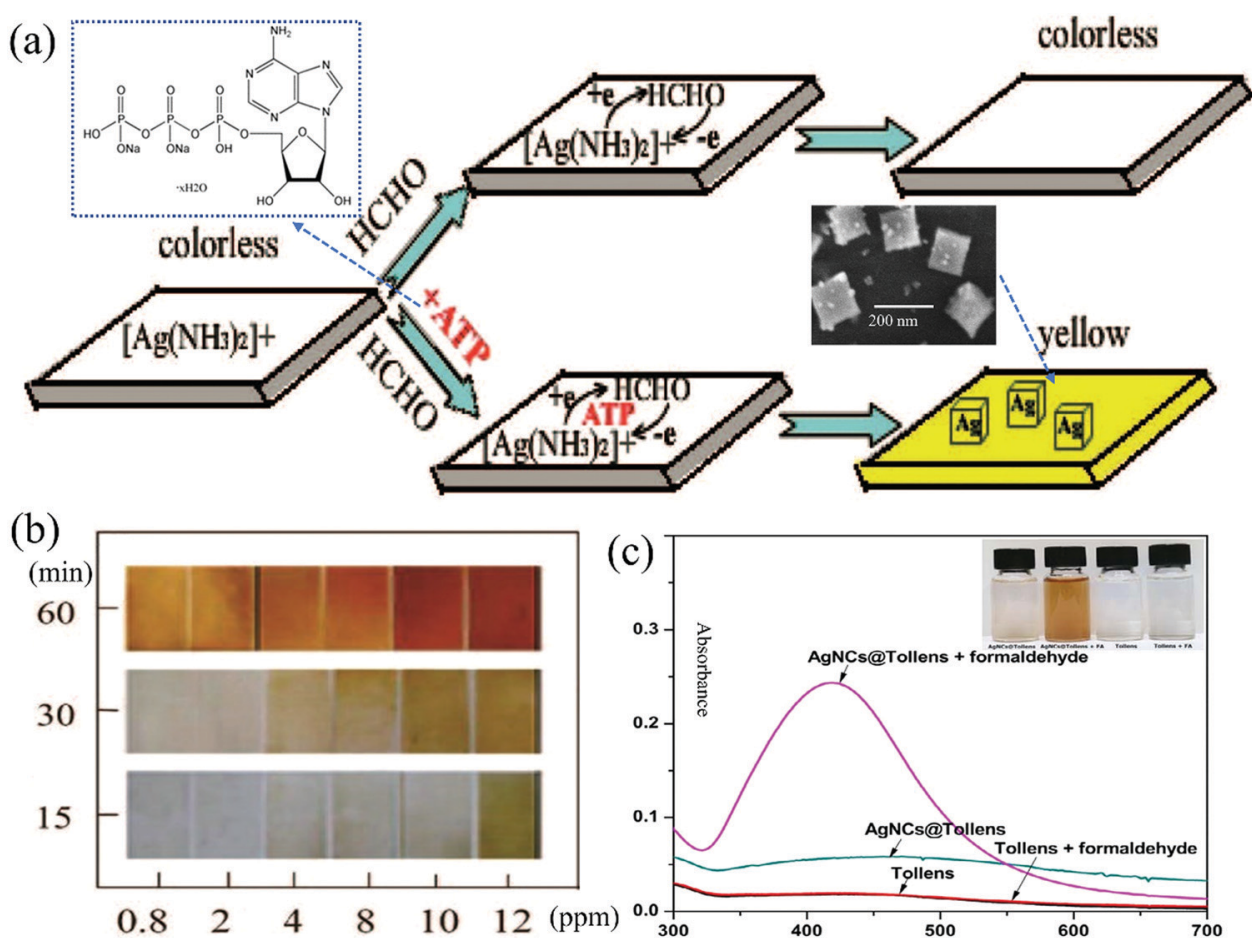

Fig. 14 (a) Schematic representation of the silver nanocubes formed on the Nafion film, and (b) the time course of color change of the Nafion film (reproduced with permission. ${ }^{90}$ Copyright: 2008, American Chemical Society). (c) Absorption spectrum of Ag NCs@Tollens' in the absence and presence of $35 \mu$ M FA (Reproduced with permission. ${ }^{91}$ Copyright: 2018, Elsevier).

the formation of the nanocube shape, while $\mathrm{NaOH}$ just supplied the alkaline environment. As shown in Fig. 14b, the color of the test strip changed with temperature and time. However, this method requires long reaction time (60 $\mathrm{min})$ and suffers from inadequate sensitivity with an LDL of $60 \mathrm{ppb}$ in the linear working range of $0.06-10 \mathrm{ppm}$ due to the weak reducing ability of FA to $\left[\mathrm{Ag}\left(\mathrm{NH}_{3}\right)_{2}\right]^{+}$. After comparison with other mediators such as cetyltrimethylammonium bromide (CTAB), sodium dodecyl sulfate, and poly(vinyl pyrrolidone), it was also found that ATP was a much better stabilizer to immobilize Ag nanocubes only on the Nafion film and not in the solution by avoiding the diffusion of the not promptly reduced $\left[\mathrm{Ag}\left(\mathrm{NH}_{3}\right)_{2}\right]^{+}$ into the solution.

To further improve the detection efficiency, Ngeontae et al. ${ }^{91}$ reported a colorimetric $\mathrm{Ag}$ nanoclusters (Ag NCs)@Tollens chemodosimeter with a very weak absorption band at approximately $350-500 \mathrm{~nm}$ corresponding to a light-yellow color. Its fabrication was based on a newly prepared Tollens' reagent in the presence of ready-made colloidal Ag NCs, and stabilized and templated by polymethacrylic acid. As shown in Fig. 14c, when the sensor was exposed to FA in an alkaline solution, a dark yellow color change occurred, accompanied by the growing average size from $0.9 \pm 0.1 \mathrm{~nm}$ of the NCs (no LSPR, size-dependent fluorescence) to $5.2 \pm 0.2 \mathrm{~nm}$ of the NPs (with an LSPR absorbance peak at $430 \mathrm{~nm}$, decreased photoluminescence) upon the deposition of $\mathrm{Ag}^{0}$ from the Tollens' reagent. An LDL of $840 \mathrm{ppb}$ was achieved under a linear working range of 900-1500 ppb. On the contrary, in the absence of the Ag NCs, only a gray precipitate of $\mathrm{Ag}$ was formed upon the Tollens' reaction. This indicated that $\mathrm{Ag}$ NCs exhibiting molecule-level size can act as crystal seeds to assist crystal growth into highquality NPs. It should be noted that the detection sensitivity highly depended on an optimized $\mathrm{pH}$ value of 4.5 , which was adjusted by the acetic-acetate buffer before the LSPR spectrum measurement of the Ag NPs.

The above colorimetric sensor based on the Tollens' reaction-mediated LSPR of Ag NPs was employed for industrial FA ( $37 \%$ in water) as the analyte. It is believed that these sensors are also feasible for gaseous aldehyde monitoring via additional solution-adsorbed trapping before detection, ${ }^{24}$ which has been widely used for gaseous FA detection including the commercial system. Moreover, similar to the Hantzsch reaction-based FA gas sensors, the solid matrices such as solgels could be utilized to immobilize another as-synthesized NPs (as the crystal seed similar to the above-mentioned AgNCs) along with Tollens' reagent for gaseous detection. Au@Ag coreshell nanostructures have been adopted due to the much abundant color variation and large optical peak shifts, which are in line with the strong color intensity change (i.e., visual resolution) than that from single $\mathrm{Ag}$ NPs' transformation caused by the combined LSPR effect of both Au and Ag NPs. ${ }^{92,93}$

The porous nature of the agarose hydrogel, which can provide a matrix to embed colloidal Au NPs (with a uniform diameter of $13.0 \pm 1.5 \mathrm{~nm}$ ) and Tollens' reagent as well as enough water to allow the Tollens' reaction. Taking advantage of agarose, Zeng et $a .^{92}$ fabricated a solid-phase agarose gel containing spherical and core-shell Au@Ag NPs (Fig. 15a). The plasmon adsorption spectra of these Au@Ag NPs highly 
depended on the shell-to-core thickness ratio, whose slight difference can lead to an apparent red-to-pink-to-yellow color change with blue-shifted plasmon maximum absorbance from $520 \mathrm{~nm}$ to $410 \mathrm{~nm}$. After exposure to aqueous FA, a distinct color change occurred from pink to deep yellow, which is related to increased plasmon absorbance at $410 \mathrm{~nm}$ as the concentration increased (Fig. 15b). The as-prepared agarose gels could be cut into small sizes with various shapes and showed the desired room-temperature colorimetric response within $1 \mathrm{~min}$ toward both aqueous and gaseous FA. The eye-distinguishable LDL was found to be $3.0 \mathrm{ppb}$ and the detectable LDL was found to be $1.5 \mathrm{ppb}$ within a linear range of $3.0 \mathrm{ppb}-1.2 \mathrm{ppm}$. A low LDL of $80 \mathrm{ppb}$ within a linear range of $80 \mathrm{ppb}-20 \mathrm{ppm}$ was recorded for the gaseous FA (Fig. 15c).

Au nanorods (NRs) possess two plasmon absorption band$\mathrm{s}$-longitudinal plasmon absorption band (LPAB) and transverse plasmon absorption band (TPAB). Thereinto, the location of LPAB mainly depends on the shape, surface charge, and dielectric conditions of the $\mathrm{Au}$ NRs. The shape effect of the nanostructured composites was also demonstrated by the core-shell Au@Ag NRs with a length of 40-50 nm, which were formed via anisotropically depositing reduced $\mathrm{Ag}^{0}$ on the surface of $\mathrm{Au} \mathrm{NRs}$ (initial length of 20-30 nm) from an aqueous solution mixture of FA and Tollens' reagent under the optimized $\mathrm{pH}$ value of 9.58 in the presence of glycine- $\mathrm{NaOH}$ as the buffer.

Liu et al. described a novel Au NRs colorimetric sensor based on the linear correlation of $\Delta \lambda_{\text {LPAB }}$ with the concentration of FA and the color change of the solution. ${ }^{93}$ The FA could reduce $\mathrm{Ag}^{+}$ to $\mathrm{Ag}$ on the surface of Au NRs to form the $\mathrm{Au}$ core-Ag shell nanorods (Au@AgNRs) in an Au NRs- $\mathrm{Ag}^{+}-\mathrm{HCHO}$ system, which caused the dielectric function to change, the LPAB of AuNRs to redshift $\left(\Delta \lambda_{\text {LPAB }}\right)$, and the color of the solution to change noticeably. A rapid colorimetric response was observed along with the transformation from the CTAB-stabilized Au NRs to the coreshell Au@Ag NRs (Fig. 15d, e) in the presence of FA. The light gray to cyan color change arose from the red-shifting of the maximum LPAB at $748 \mathrm{~nm}$ and TPAB at $555 \mathrm{~nm}$ (Fig. 15f). Similar to the role of ATP and polymethacrylic acid toward the shape- and sizecontrollable Ag nanocubes or NCs, ${ }^{90,91}$ the presence of CTAB was also critical to the increased aspect ratio (length/width) by adjusting the dielectric environment around the Au NRs. (a)

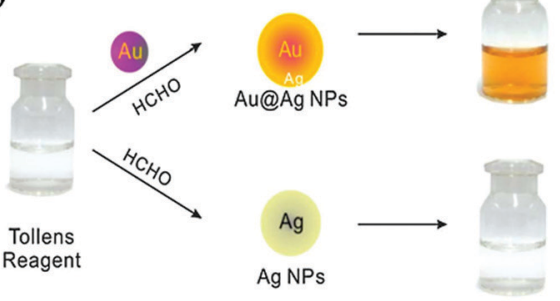

(d)

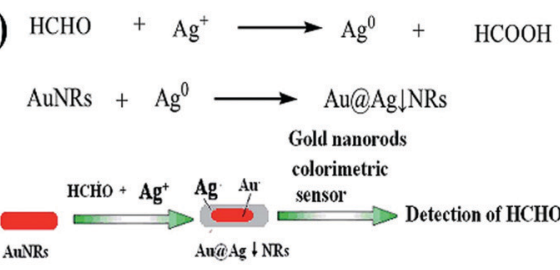

(g)

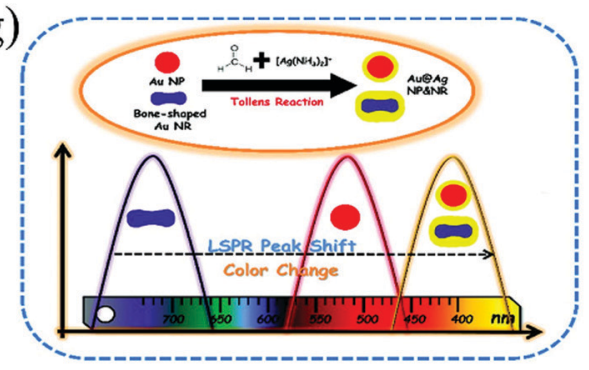

(b)
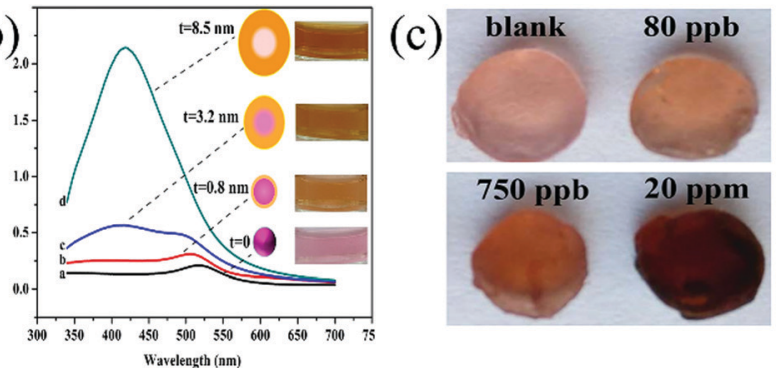

(e)
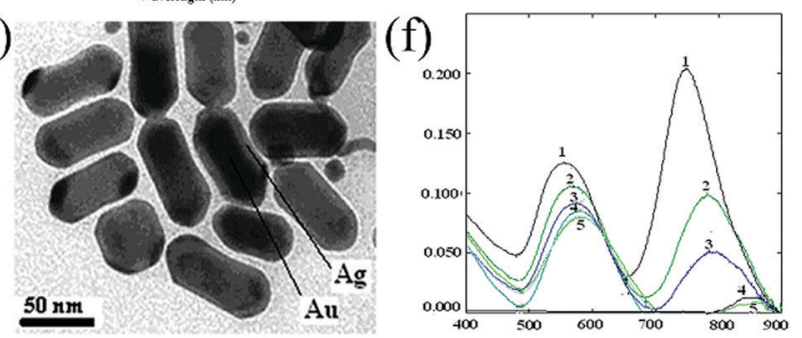

(i)

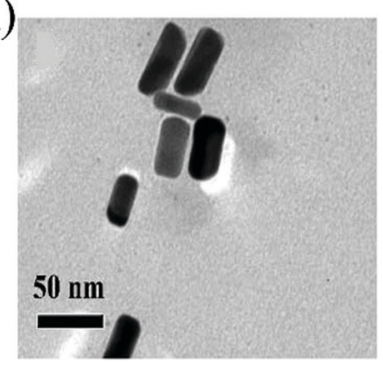

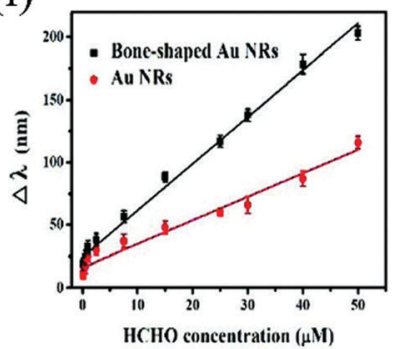

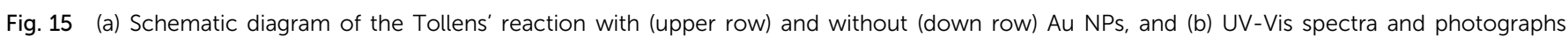

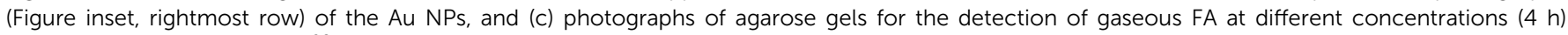

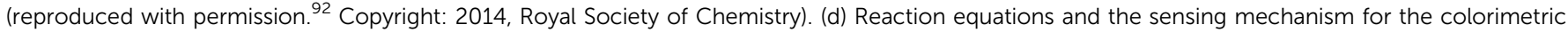

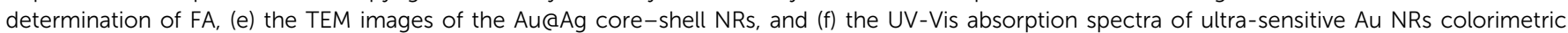

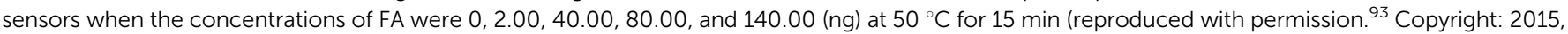

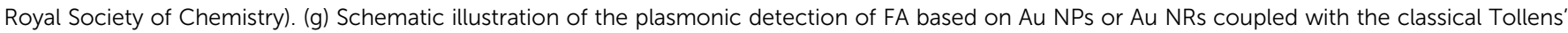

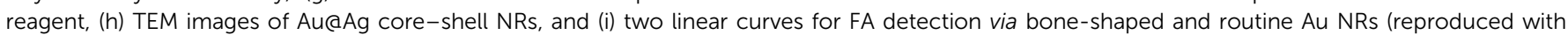
permission. ${ }^{94}$ Copyright: 2019, Royal Society of Chemistry). 
In another work reported by Duan et al. in $2019,{ }^{94}$ boneshaped Au@Ag core-shell NRs enabled the spectrophotometric (plasmon absorption) and naked-eye detection of FA (Fig. 15g and $\mathrm{h}$ ). The monodispersed cetyltrimethylammonium chloride (CTAC)-capped bone-shaped Au NRs with a length of ca. $55 \mathrm{~nm}$ and a diameter of $c a .20 \mathrm{~nm}$ and a Tollens' reagent was used for the colorimetric assay sensor design for FA measurement. Due to the unique bone-shaped structure, the preferential deposition of reduced $\mathrm{Ag}^{\mathrm{O}}$ occur onto the concave section of $\mathrm{Au}$ NRs. These Au NRs exhibited not only two main plasmon absorption bands at about $520 \mathrm{~nm}$ and $715 \mathrm{~nm}$ that arose from the transverse and longitudinal LSPR, respectively, but also a small characteristic peak of the bone-shaped $\mathrm{Au}$ NRs at about $575 \mathrm{~nm}$. After interaction with FA in solution, the LPAB shifted from $715 \mathrm{~nm}$ to $500 \mathrm{~nm}$ with the emergence of a new absorption band at about $400 \mathrm{~nm}$, almost covering the full visible region. Concurrently, the color changed apparently from light gray to dark blue, purple, red, orange, and finally to yellow. The sensor could detect FA at an LDL of $0.2 \mathrm{ppb}$ within a wide linear range (3-1500 ppb) (Fig. 15i). Anisotropical shell-to-core deposition and the use of CTAC (not CTAB) were two critical issues for such an extremely rapid and sensitive response due to the accelerated Tollens' reaction and dramatic morphology changes of NRs.

Much recently, based on size-controlled Au NPs seeds for Tollens' reaction (Fig. 16a), Huo et al. ${ }^{95}$ further developed a 3-channel colorimetric sensor array for the determination of 10 aldehydes (Fig. 16b) at low concentrations with the help of $\Delta$ RGB-model optical signal capture, followed by data analysis using PCA and the pattern recognition method (Fig. 16c). Three negatively charged Au NPs with different particle diameters $(13,22$, and $40 \mathrm{~nm})$ were selected, which could significantly increase to $27 \mathrm{~nm}, 40 \mathrm{~nm}$, and $50 \mathrm{~nm}$ for the Au@Ag core-shell NRs, respectively, after exposure to model FA with an LDL of $0.9 \mathrm{ppb}$. The 3-channel array originated from the different plasmon absorption peaks at 517, 529, and $532 \mathrm{~nm}$, corresponding to the three sizes of Au NPs, respectively. For each aldehyde, the discrimination sensitivity could reach $100 \%$

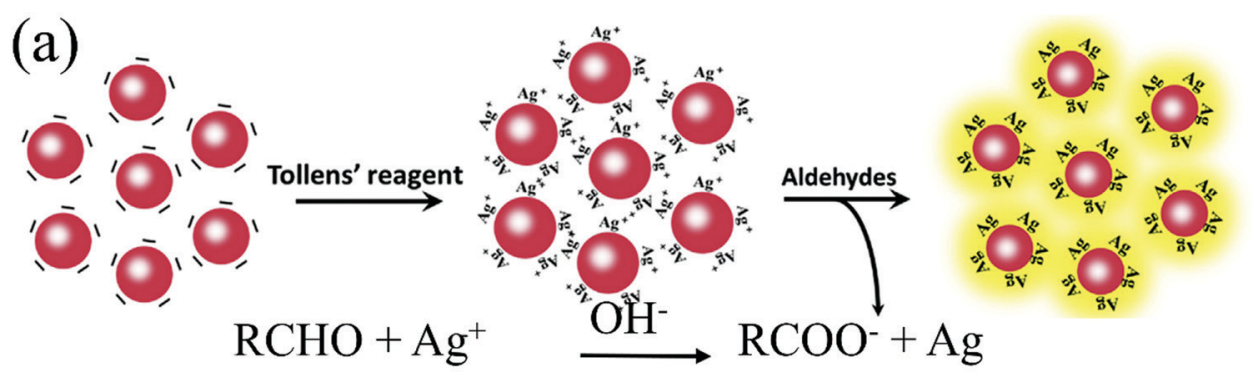

(b)
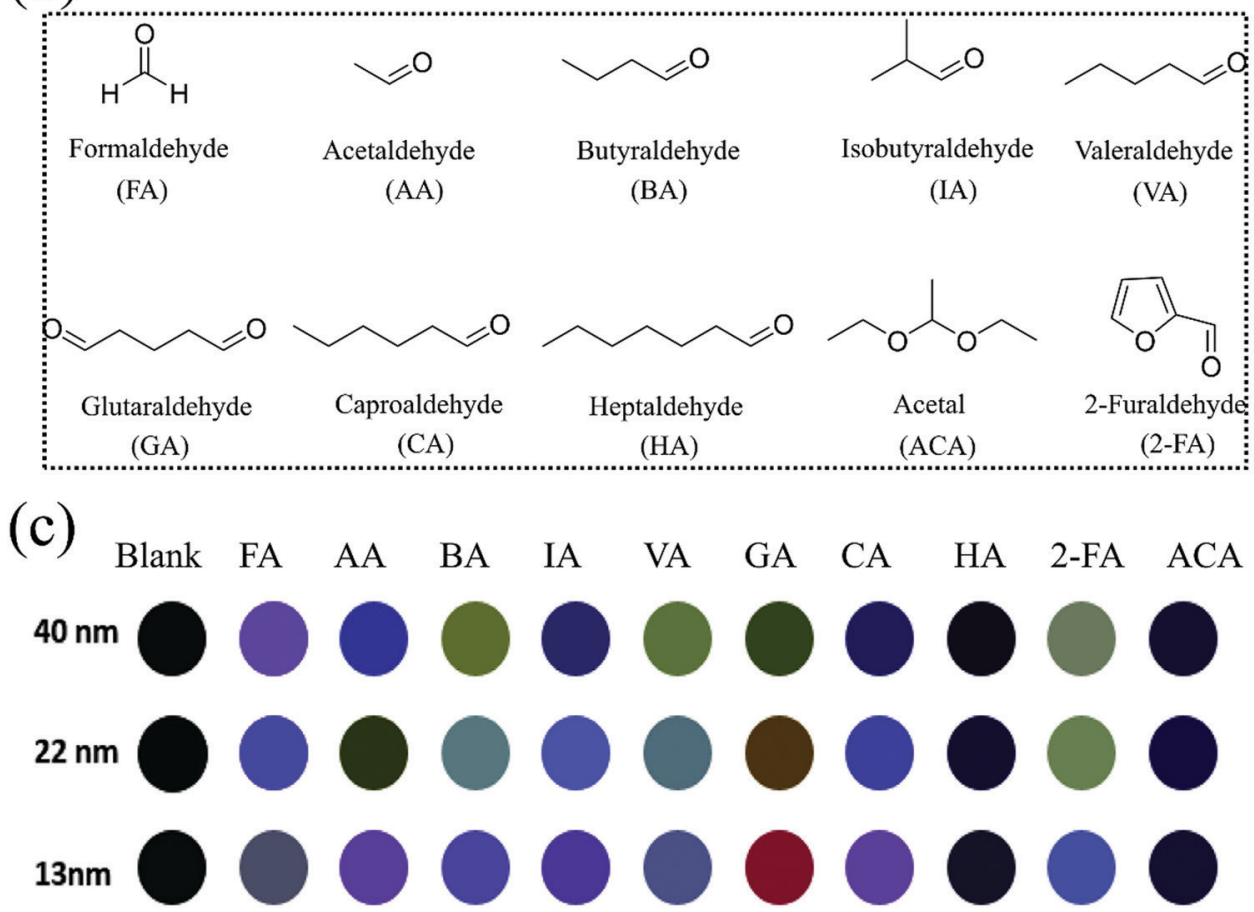

Fig. 16 (a) Schematic of the colorimetric sensing mechanism of Au NPs toward aldehydes, (b) chemical structures of the targeted 10 aldehydes, and (c) actual difference maps of the sensor arrays for 10 aldehydes at the concentration of $30 \mathrm{ppb}$ and the blank sample (in the absence of aldehyde) (reproduced with permission. ${ }^{95}$ Copyright: 2020, Elsevier). 
(except for butyraldehyde), and the recognition accuracy was above $90 \%$.

\section{Fluorometric sensors}

Compared to naked-eye-visual colorimetric methods, fluorometric chemosensors exhibit relatively rapid response and high sensitivity for the detection of trace gaseous analytes under UV light irradiation. ${ }^{34,60,96,97}$ According to fluorometric chemosensors for detecting aldehydes, this paper divides the reaction mechanism into four categories, including aldimine condensation, ${ }^{98-105}$ Aza-Cope rearrangement, ${ }^{106-109}$ QDs adjustment, ${ }^{110,111}$ and functional MOFs interaction. ${ }^{112,113}$ Most of these reactive fluorescent probes have been applied to detect aldehydes through the chemical reaction between the - $\mathrm{CHO}$ group and the amine group on the probe. A summary and comparison of these sensing mechanisms and the corresponding probing materials, substrates, as well as the detection results (selectivity, LDL, response time, switching type, etc.) will be discussed in the following section in line with a brief list in Table 2.

\subsection{Sensors based on aldimine condensation}

Similar to colorimetric chemosensors, a large number of fluorometric chemosensors can also be realized via the aldimine condensation reaction between the aldehyde and the amine group or hydrazine $\left(-\mathrm{NH}-\mathrm{NH}_{2}\right)$. A nonfluorescent or weakly fluorescent probe forms formimine or methylenehydrazine, which leads to turn-on fluorescence signal due to the adjusted charge transfer process, photoinduced electron transfer (PET), or intramolecular charge transfer (ICT). This kind of probe generally consist of two parts: an energy-matched fluorophore and a receptor, which can be regarded as a fluorescence quencher due to the strong electron-donating effect of the amino or hydrazine functional group to the conjugated core. In addition to these sensing methods based on aggregation induced quenching (ACQ) probing molecules, the fluorescence response based on aggregation-induced emission (AIE) adjusted by the aldimine condensation reactions will also be discussed.

3.1.1 7-Nitrobenz-2-oxa-1,3-diazole (NBD) derivatives. As a fluorophore, 7-nitrobenz-2-oxa-1,3-diazole (NBD) has a high quantum yield in organic solvents or membranes and its chlorine and fluorine derivatives can be easily substituted with the amine groups. ${ }^{114}$ The NBD-derivatives grafted with $-\mathrm{NH}_{2} /-$ $\mathrm{NH}-\mathrm{NH}_{2}$ show no or weak fluorescence due to the PET effect between $-\mathrm{NH}_{2} /-\mathrm{NH}-\mathrm{NH}_{2}$ and the fluorophore. When aldehyde reacts with the amine groups on the NBD-derivatives via aldimine condensation, the PET process is inhibited, leading to the fluorescence enhancement (turn-on process), which shows the potential of NBD-derivatives in the field of gaseous aldehyde detection by attaching them to the solid substrate.

To develop a fluorometric chemosensor for the real-time and sensitive detection of FA vapor, Mahapatra et al. ${ }^{98}$ synthesized NBD-piperazine conjugate (NPC) probe (Fig. 17a) with PET-induced fluorescence quenching by bridging 2-aminoethyl piperazine to NBD. When aldimine condensation occurred, the PET process from 2-aminoethyl piperazine to NBD stopped and resulted in a turn-on fluorescence signal. The NPC showed good sensitivity in liquid FA detection with an LDL of $2.52 \mathrm{ppb}$ (also a linear range of $0-75 \mathrm{ppb}$ ). In addition, the NPC probe also showed excellent selectivity for FA under the presence of other aldehydes (AA, methylglyoxal, $p$-nitrobenzaldehyde). The strong condensation between FA and NPC was also helpful for the detection from weak acid to neutral $\mathrm{pH}$. However, the research on the concentration determination of gaseous FA was

Table 2 Comparison of fluorometric chemosensors fabricated from different materials

\begin{tabular}{|c|c|c|c|c|c|}
\hline Probing material & Aldehyde & $\mathrm{LDL}(\mathrm{ppb})$ & Response times (min) & Switching type & Ref. \\
\hline Test paper kits with NPC & FA & 2.52 & 10 & Turn-on & 98 \\
\hline FAP & FA & 12 & 30 & Turn-on & 99 \\
\hline NA3 & FA & - & - & Turn-on & 100 \\
\hline CNC-ANH & FA & 438 & - & Turn-on & 102 \\
\hline DP5J-Bi & FA & 0.1 & 0.125 & Turn-on & 105 \\
\hline Filter paper strips with HBT-FA & FA & - & 60 & Turn-on & 106 \\
\hline FA-P & FA & - & 30 & Turn-on & 107 \\
\hline TPE-FA & FA & 25.2 & - & Turn-on & 108 \\
\hline TP-FA & FA & 15.3 & 45 & Turn-on & 109 \\
\hline QDMF & FA & 1 & 120 & Turn-off & 110 \\
\hline CdTe QDs (array) & FA & 80 & - & Turn-off & 139 \\
\hline CdSe@ZnS QDs & FA & 500 & 1.7 & Turn-off & 111 \\
\hline $\mathrm{CDs@SiO}{ }_{2}-\mathrm{NH}_{2}-\mathrm{Ag}^{+}$ & FA & 3 & 30 & Turn-off & 140 \\
\hline LTW & FA & 600 & - & Turn-on & 141 \\
\hline NGQDs & FA & 4.5 & - & Turn-off & 143 \\
\hline \multirow[t]{3}{*}{$\mathrm{Eu}^{3+}$-Functionalized ZnO@Uio-MOFs } & FA & 42 & - & Turn-on & 157 \\
\hline & $\mathrm{AA}$ & 58 & & & \\
\hline & Acraldehyde & 66 & & & \\
\hline Dual-emissive $4 \mathrm{~d}-4 \mathrm{f} \mathrm{Ag}^{+}-\mathrm{Eu}^{3+} \mathrm{MOF}$ & FA & 51 & 60 & Turn-on & 158 \\
\hline $\mathrm{Eu}\left(\mathrm{NO}_{3}\right)_{3} 6 \mathrm{H}_{2} \mathrm{O}$ & FA & 11.8 & - & Turn-on & 159 \\
\hline Cu-BTC MOFs & FA & 18.67 & 1 & Turn-off & 113 \\
\hline $\mathrm{Al}^{3+}$-MOFs with $\mathrm{H}_{2} \mathrm{BDC}-\mathrm{N}_{2} \mathrm{H}_{3}$ & FA & - & 20 & Turn-on & 103 \\
\hline $\mathrm{Cd}^{2+}$-Based MOFs & FA & 78000 & - & Turn-on & 160 \\
\hline Chair-conformation resorcin[4]arene & Aldehydes & - & - & Turn-on & 161 \\
\hline
\end{tabular}




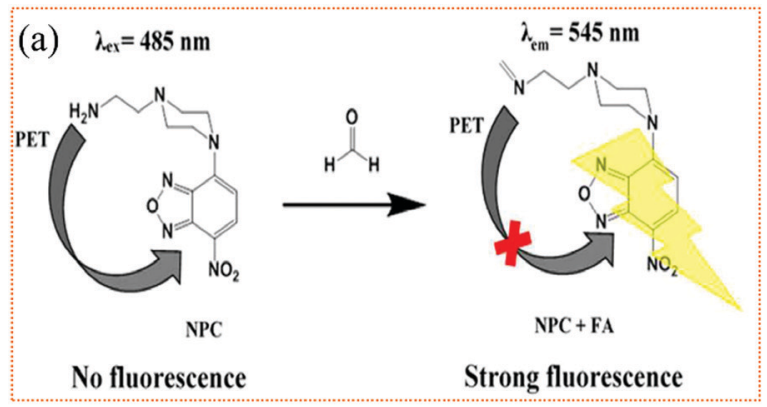

(b)

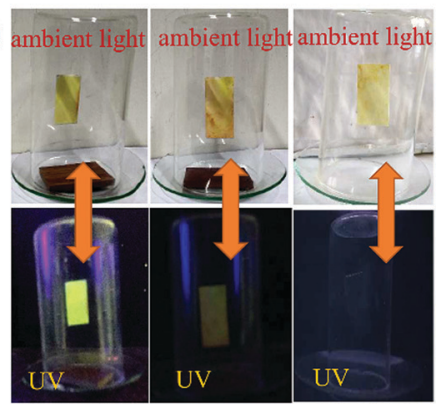

(c)

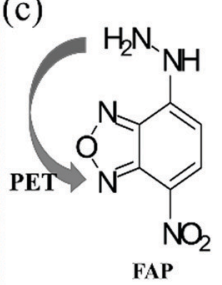

Weak fluorescence

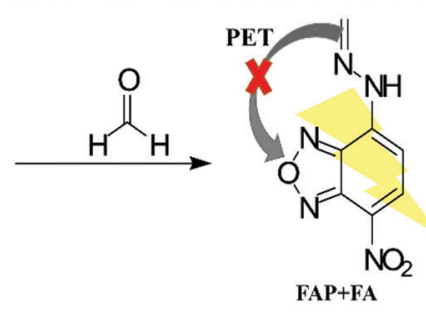

Strong fluorescence (d)

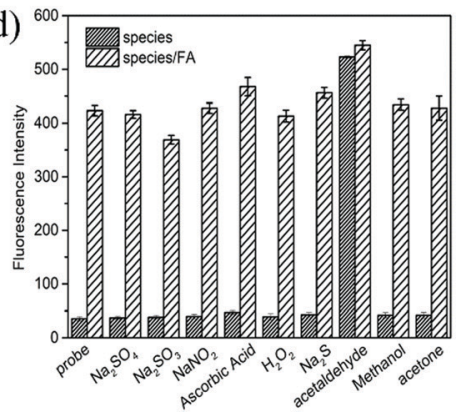

Fig. 17 (a) The proposed sensing mechanism of NPC for FA, and (b) the photographs of the TLC strips under NPC in the presence of new plywood, one month old plywood, and without substrate (reproduced with permission. ${ }^{98}$ Copyright: 2018, Royal Society of Chemistry). (c) The proposed sensing mechanism of FAP for FA, and (d) the selectivity and anti-interference effect of the FAP for FA (reproduced with permission. ${ }^{99}$ Copyright: 2020 , Elsevier).

not further discussed; they just used thin-layer chromatography (TLC) silica gel plate as the substrate to prove the feasibility of detection, as shown in Fig. 17b.

The hydrazine group can also act as a fluorescence quencher for NBD based on the PET process, which can be employed for the selective and fast detection of aldehydes at room temperature. Recently, Wang et al. ${ }^{99}$ synthesized a probe molecule, FAP (Fig. 17c), which shows significant turn-on fluorescence response to FA vapor in the range of $12-640 \mathrm{ppb}$. The effect of $\mathrm{pH}$ on the fluorescence intensity was significant, especially in the low $\mathrm{pH}$ region $(\mathrm{pH}=2.48-4.55)$. However, sodium sulfate $\left(\mathrm{Na}_{2} \mathrm{SO}_{3}\right)$ would also have an influence on the accuracy detection in the experiment (Fig. 17d) because FA and sodium hydrogen sulfite would undergo the nucleophilic addition reaction to form $\mathrm{CH}_{2}(\mathrm{OH}) \mathrm{SO}_{3} \mathrm{Na}$.

3.1.2 Naphthalimide derivatives. Another familiar fluorophore for chemosensors is naphthalimide and its derivatives, which benefit from their excellent properties such as optical stability, large Stokes' shift, strong absorption, and UV-Vis emission. ${ }^{115}$ In recent years, some researchers have developed a series of probes for the detection of FA using naphthalimide derivatives as the fluorophore and amine/hydrazine as the receptor as well as fluorescence quencher, relying on the PET or ICT effect. ${ }^{100,102}$

Sun et al. ${ }^{100}$ reported a sensitive probe based on 1,8naphthylamino fluorometric derivatization reagent (NA3) utilizing hydrazine as the reaction site, which can rapidly detect $\mathrm{FA}$ at room temperature within $9 \mathrm{~min}$. When the structure NA3 + HCHO was formed along with aldimine condensation, the PET process was inhibited by the protonation of the tertiary amine group, resulting in a "turn-on" fluorescent signal (Fig. 18a).
While the ICT process (the imine moiety and amino group located at the 4-position of the 1,8-naphthalimide unit to 1,8-naphthalimide) was carried out, the fluorescence was further enhanced. The sensor showed excellent selectivity toward FA in the presence of other interfering compounds (benzene, acetone, 2,4-dihydroxybenzaldehyde, terephthalaldehyde, 4-methoxybenzaldehyde, trichloroacetic aldehyde, 4-hydroxybenzaldehyde). As shown in Fig. 18b, the test paper also showed turn-on fluorescence signal in the presence of FA. However, LDL in the gas phase was not discussed in detail.

As we know, FA always coexists with water molecules in a humid environment, and the change in the RH makes chemosensors unable to measure the concentration of FA accurately, which greatly limits the practical application of the sensor. In order to reduce the influence of $\mathrm{RH}$, a chemosensor that can simultaneously detect the $\mathrm{RH}$ and FA concentration was reported by Gao et al. ${ }^{102}$ A kind of naphthalimide derivative containing hydrazine and cationic groups, [N-(3-N-benzyl$N, N$-dimethylpropyl ammonium chloride)-1,8-naphthalimide]hydrazine (ANH), was assembled on the surfaces of the CNCs, which had numerous sulfate groups to form the CNC-ANH membranes (Fig. 18c). Notably, ANH molecules were welldispersed on the surface of the CNCs, which prevented the AIE effect of the molecules caused by the $\pi-\pi$ stacking interaction among the fluorophores, and further improved the off-on sensitivity. When the membrane was exposed to FA vapor, the PET process was inhibited and provided a "turn-on" fluorescent signal (Fig. 18d). The membrane had an LDL of $438 \mathrm{ppb}$ FA at $65 \% \mathrm{RH}$. Besides, it was also proved that the functional CNCs membrane had great application value as the substrate for small-molecule signal probe. 
(a)

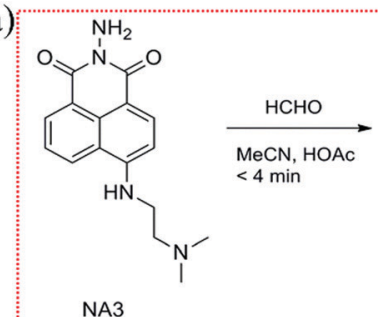

NA3

(c)

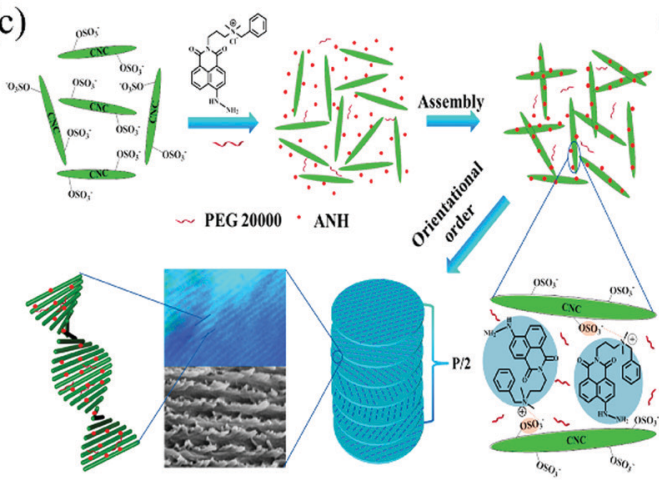

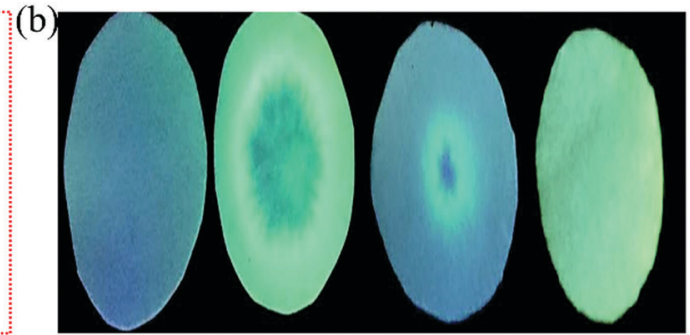

(d)

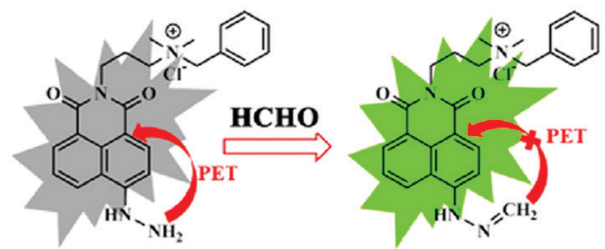

Weak Fluorescence Strong Fluorescence
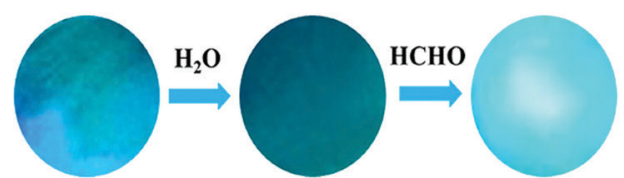

Fig. 18 (a) Schematic diagram of the mechanism for detecting FA by NA3, and (b) photographs of the test paper in the absence and presence of FA under 365 nm ultraviolet light (blank; FA in acetonitrile; FA in water; FA in air) (reproduced with permission. ${ }^{100}$ Copyright: 2016 , Royal Society of Chemistry), (c) formation process of functionalized fluorescent photonic crystal films, and (d) the schematic diagram of the mechanism for detecting FA by ANH and the photographs of the membrane (reproduced with permission. ${ }^{102}$ Copyright: 2020, American Chemical Society).

3.1.3 Pillar $[n]$ arene derivatives. Most of the fluorometric probes for aldehyde detection are composed of fluorophores with the characteristics of ACQ. When fluorescent sensor molecules with ACQ features are loaded on the solid substrate to detect FA gas, there will always be some fluorophores in the aggregation state emitting weak fluorescence, thus representing the weak detection sensitivity. Therefore, ACQ-based probes need to be dispersed in solvents, which are often used to detect aldehydes in solution. In comparison, the AIE fluorophores emit strong fluorescence in the aggregation state. ${ }^{116}$ Therefore, the AIE fluorophore is an ideal candidate for the design of solid probes, which can realize the simple and direct detection of gaseous aldehydes. ${ }^{117}$

Pillar[n]arenes, a new kind of cylindrical macrocyclic host, has been widely used in the fields of chemistry and biomedical materials due to its easy synthesis, unique columnar structure, multiple functions, interesting host-guest properties, and unique supramolecular assembly characteristics. In addition, the unique molecular structure of pillar $[n]$ arenes leads to various supramolecular assembly driving forces, including $\mathrm{C}-\mathrm{H}-\pi, \pi-\pi$, cation $-\pi$, and hydrophobic/hydrophilic, which provides a novel platform for the construction of various interesting supramolecular systems, as well as AIE-based chemosensors. ${ }^{118-121}$ An AIE-based sensor ${ }^{105}$ with an LDL of $0.1 \mathrm{ppb}$ toward FA in $7.5 \mathrm{~s}$ was developed by introducing hydrazide groups into pillar[5] arene (DP5J) (Fig. 19a). In order to improve the aldimine condensation reaction rate, a Lewis acidic metal triflate $\left(\left(\mathrm{CF}_{3} \mathrm{SO}_{3}\right)_{2} \mathrm{Bi}\right)$ was introduced as the catalyst. ${ }^{122}$ The test kit was prepared by loading the catalyst $\left(\mathrm{CF}_{3} \mathrm{SO}_{3}\right)_{2} \mathrm{Bi}$-preplaced DP5J sensor onto a silica gel plate. As shown in Fig. 19a and b, DP5J reacted with FA to produce a carbinolamine intermediate (DP5J$\mathrm{OH})$ via aldimine condensation and then produce an imine product (DP5J-S) via dehydration. Subsequently, due to intermolecular hydrogen bonds between the neighboring DP5J-OH and DP5J-S, the AIE phenomenon occurred through the aggregation of $\pi-\pi$ interaction, resulting in turn-on fluorescence. In addition to the excellent sensitivity toward FA, the probe also showed good selectivity toward FA in the presence of other miscellaneous competitive aldehydes including benzaldehyde, salicylaldehyde, and $p$-fluorobenzaldehyde. AIE-based response is quite faster than previous chemosensors, relying on the ACQ-type organic fluorophores. Although the molecular design and synthesis of AIE-type fluorophores are more complex than the easily achieved ACQ-type dyes, it is believed that further breakthroughs will be made in AIE fluorophores with great efforts, which may broaden the horizon of supramolecular chemistry.

\subsection{Sensors based on Aza-Cope rearrangement}

The fluorescent probes mentioned in the previous section can react with - CHO by directly modifying with the amino groups, hydrazine, etc., on their main chains and trigger the change in the fluorescence of the probe molecule through various mechanisms (PET, ICT, tunable aggregation, etc.). However, any attempt to improve the sensitivity and selectivity of optical chemosensors toward the aldehydes often interferes with the photophysical properties of the fluorophore cores themselves. It more or less limits the choice of the skeleton structures of the fluorophores, as shown in Section 3.1. Thus, it is necessary to develop another method to modify the probes without affecting the photophysical properties of the conjugated fluorophore 


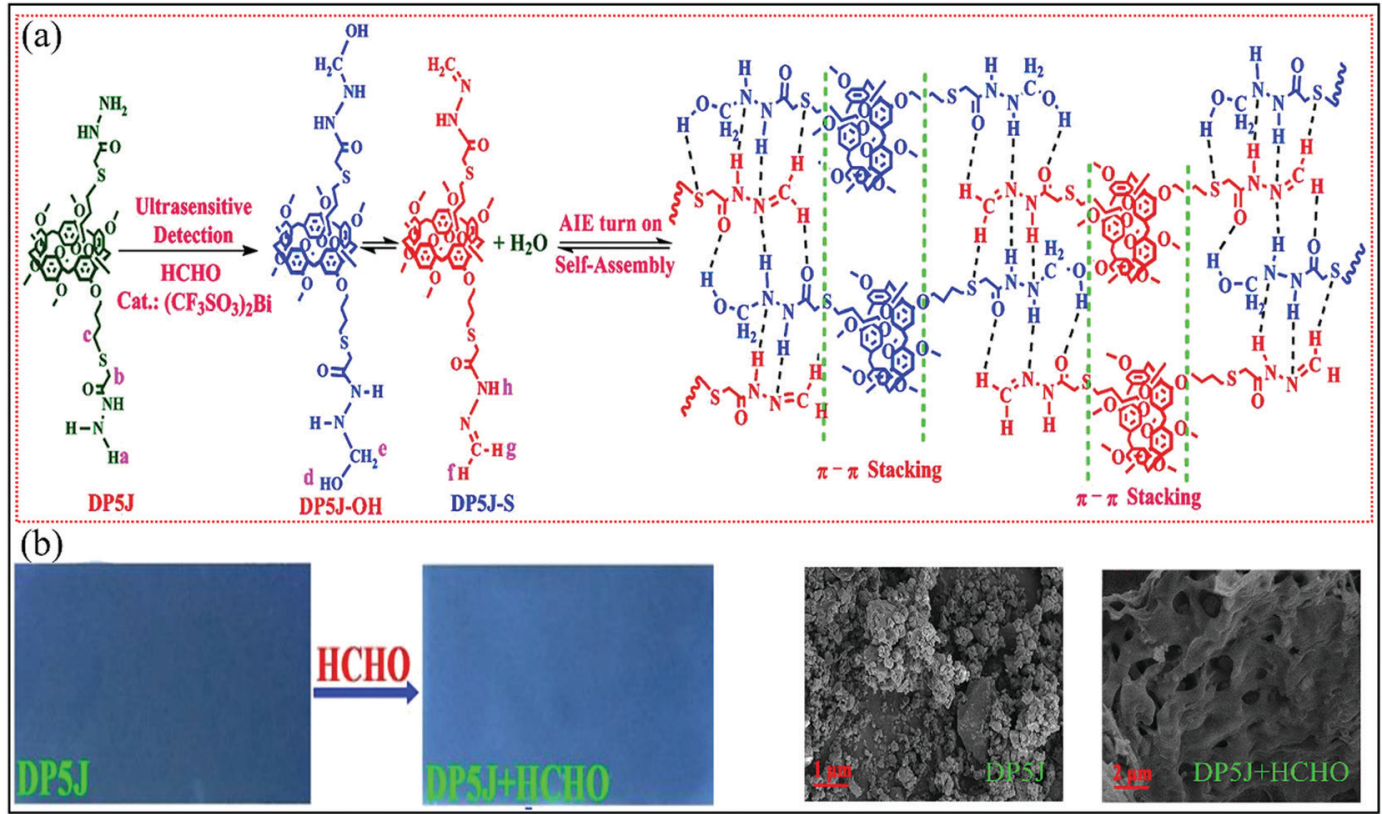

Fig. 19 (a) Proposed recognition mechanism of DP5J for monitoring FA, and (b) fluorescence color changes of silica gel plates and SEM of DP5J (amorphous powder) and DP5J + HCHO (cross-linked network structure) (reproduced with permission. ${ }^{105}$ Copyright: 2018, American Chemical Society).

cores in the presence of fluorescence excitation. In this case, Aza-Cope rearrangement has been demonstrated to provide more choices of fluorophores for the detection of FA. ${ }^{123}$ It can be attributed to a self-immolative $\beta$-elimination linker, which can be installed into any fluorophore containing a common phenolic group. In addition, the Aza-Cope rearrangement exhibits high selectivity for FA over other reactive carbonyl species and carbonyl species, such as acetaldehyde, methylglyoxal, and 4-hydroxynonenal. ${ }^{30,124}$ Other available fluorophores can also provide more options for substrates, which is more convenient for the construction of portable solid-state sensors for the selective and sensitive detection of gaseous FA.

Based on the ICT effect induced by the Aza-Cope rearrangement leading to a significant red shift, Zheng et al. ${ }^{106}$ synthesized a fluorescent probe (HBT, Fig. 20a) using 2-(2-hydroxyphenyl)benzothiazole, which showed high selectivity and quick fluorescence response to FA. As shown in Fig. 20a and b, HBT reacted with FA to form HBT-HCHO via the Aza-Cope rearrangement.
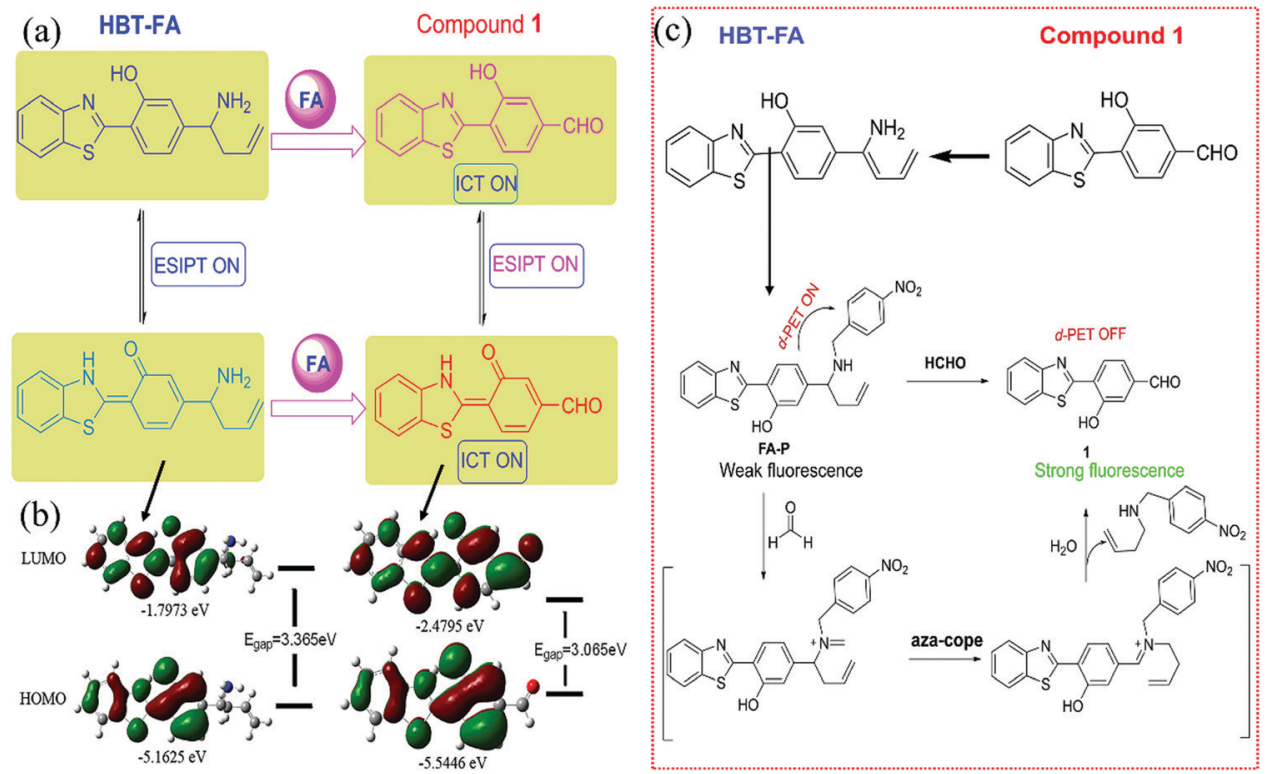

Fig. 20 (a) Scheme of the reaction between HBT and FA, and (b) the HOMO-LUMO energy level and the electron distribution of HBT and HBT-HCHO (reproduced with permission. ${ }^{106}$ Copyright: 2018, Elsevier). (c) Synthetic route of FA-P and its reaction with FA (reproduced with permission. ${ }^{107}$ Copyright: 2019, Elsevier). 
Strong ICT effect was obtained between the electron-deficient aldehyde group formed in HBT-HCHO and the fluorophores (fluorophore to the aldehyde group), leading to a red-shift of the fluorescence bands. FA in the serum could be detected by this probe in the range of $0-300$ ppm through visible fluorescence change $(365 \mathrm{~nm})$. In the presence of other interfering gases such as acetone, butyaldehyde, and glucose, the probe displayed only negligible fluorescence change, which indicated the good selectivity for FA. However, for gaseous FA detection, a fluorescence change from blue to yellow within $1 \mathrm{~h}$ was verified by only one substrate (filter paper strip) and no data of the LDL was reported. Subsequently, they ${ }^{107}$ modified HBT with 4-nitrobenzyl ( $\left.\mathrm{Ph}-\mathrm{NO}_{2}\right)$, which possesses strong electron donor effect to synthesize a more sensitive probe (FA-P) for FA detection (Fig. 20c). The fluorescence quenching of FA-P is attributed to the PET effect from the donor 2-(2-hydroxyphenyl) benzothiazole skeleton to the receptor (nitrophenyl). When the probe reacted with FA via Aza-Cope rearrangement, the fluorescence was turned on. FA-P had excellent selectivity for FA in the presence of other aldehydes. However, only the feasibility of gaseous detection was confirmed, and no LDL was reported.

In contrast to the above 2-(2-hydroxyphenyl) benzothiazole as the ACQ-type molecules, fluorophores such as tetraphenylethylene and triphenylamine are more suitable to design portable sensors for gaseous aldehydes due to the AIE effect.

Recently, by combining the homoallylic amine group and the fluorescence quenching group (4-nitrobenzyl $\left(\mathrm{Ph}-\mathrm{NO}_{2}\right)$ ) on tetraphenylethylene, Yin et al. ${ }^{108}$ synthesized a probe (TPE-FA) with PET effect-induced turn-off fluorescence sensor for FA (Fig. 21a). TPE-FA was directly loaded on a high performance thin-layer chromatography silica gel plate to fabricate the test plate. Initially, the test plate showed weak fluorescence due to the PET process between the electron acceptor $\left(\mathrm{Ph}-\mathrm{NO}_{2}\right)$ and the electron donor (TPE-HA- $\mathrm{NH}_{2}$ ) (Fig. 21b). After the homoallylic amine group reacted with FA via 2-Aza-Cope rearrangement, the inhibited PET resulted in turn-on fluorescence. The test plate showed sensitive detection toward gaseous FA with an LDL of $25.2 \mathrm{ppb}$ in the range of $20-30{ }^{\circ} \mathrm{C}$ and $25-40 \% \mathrm{RH}$. In addition, the probe also showed high selectivity toward FA in the presence of other aldehydes such as AA, propylaldehyde, benzaldehyde, and methylglyoxal.

Another familiar fluorophore for chemosensors is triphenylamine and its derivatives, which can be used as a strong electron donor with an AIE-type skeleton. Based on their good light stability, chemical stability, and feasible modification, Li et al. ${ }^{109}$ reported an AIE-based probe (TP-FA) for FA detection, which showed color change from blue to green due to the AIE and ICT effects (Fig. 21c). The probe, which was dyed on the cotton, displayed the automatic and intelligent sensing of gaseous FA with an LDL of $15.3 \mathrm{ppb}$ in $45 \mathrm{~min}$ because of the fluffy structure of cotton. In addition, the mechanical properties of cotton were better than the test paper due to its higher shear and tensile resistance. Initially, cotton showed a deep blue fluorescence under $365 \mathrm{~nm}$ light irradiation due to the AIE feature of the
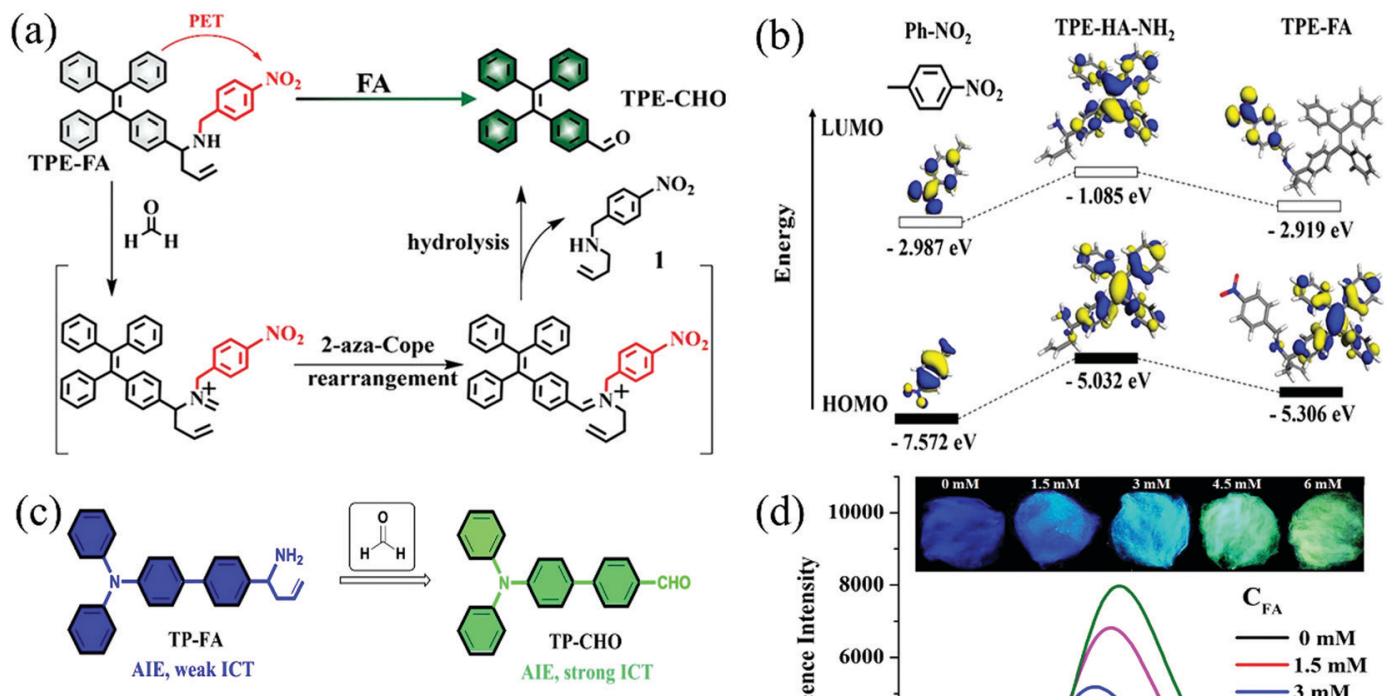

(d)

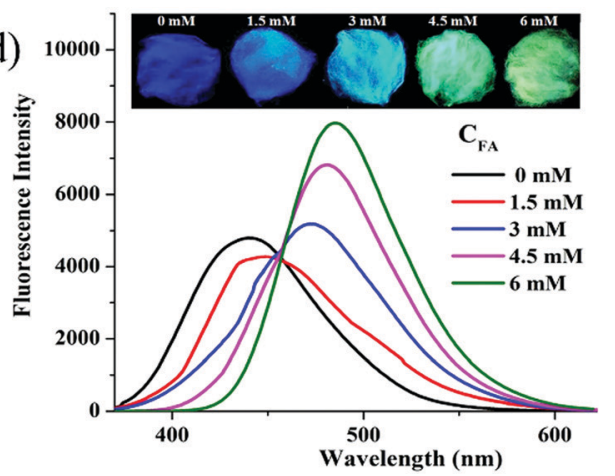

Fig. 21 (a) Schematic diagram for the detection mechanism for FA, and (b) theoretical calculations of the HOMO/LUMO energies of the compound (Reproduced with permission. ${ }^{108}$ Copyright: 2018, American Chemical Society), (c) The detection mechanism of probe TP-FA toward FA, and (d) the fluorescence spectra of the test cottons treated with different concentrations of FA. Inset: The photos of the test cottons treated with different concentrations of gaseous aldehyde (reproduced with permission. ${ }^{109}$ Copyright: 2019, Elsevier). 
fluorophores. When reacted with FA, TP-FA generated TP-CHO via 2-Aza-Cope rearrangement, which propelled the progress of ICT and resulted in a red-shift in the emission spectrum. As a result, blue fluorescence was changed to green. As shown in Fig. 21d, with the increase in the FA concentration, the emission peak of cotton gradually shifted to red. Besides, in the presence of other volatile aldehydes (including AA, propylaldehyde, butyraldehyde, isovaleraldehyde), alkaline vapor, and acidic vapor, TP-FA showed high selectivity toward FA.

\subsection{Quantum dots (QDs)}

Compared with organic fluorescent dyes, QDs have excellent chemical and optical properties (wide excitation spectra, narrow emission spectra, long fluorescence lifetime, precisely tunable emission wavelength, negligible photobleaching, etc.) and large surface for functionalization, ${ }^{125-127}$ and have been widely used in the field of fluorometric sensing. Inorganic QDs are mainly metal nanoparticles that exhibit different fluorescence properties from macroscopic materials due to quantum confinement and surface effects. ${ }^{128}$ These type of QDs are generally composed of hybrid metals with II-VI, III-V, IV-VI, or I-VII form, while the most commonly used groups are II-VI (CdSe, CdTe, CdS, etc.) and III-V (InP, InAs, etc.), which can receive excitation light and produce visible and near-infrared fluorescence. ${ }^{129}$ Carbonic QDs (CDs) are usually 0D carbon $(d \leq 10 \mathrm{~nm})$ doped with one or more atoms such as nitrogen, sulphur, and other atoms. ${ }^{130}$ In general, CDs possess several advantages that promote their application in optical chemosensors; the advantages include excellent optical properties, high photostability, resistance against chemical or photobleaching, high biocompatibility, and low cytotoxicity. Instead of electronic transitions from one valence orbital to another, quantum-dot fluorescence involves electron excitation from the bulk valence band of the semiconductor or carbon material across the energy gap, making it a conduction electron and leaving behind a hole. When the electron-hole pair eventually recombines, a characteristic photon is emitted. Small changes in the size of the nanocrystal alter the energy bandgap, thus determining the wavelength of the fluorescence. ${ }^{131}$ The sensing mechanism based on QDs is to change the fluorescence intensity of QDs through the interaction between the analyte and the recognition groups on the surface of QDs, such as excited state reaction, molecular rearrangement, and energy transfer. QDs have been widely used in biological/chemical sensing, biomedical imaging, and other fields, ${ }^{132-134}$ such as metal ion detection, enzyme activity and protein detection, and small molecule compound detection. The QD-based chemosensors usually have QDs loaded on glass because the $\mathrm{Si}-\mathrm{O}$ tetrahedral structure in glass contributes to providing a stable environment for the formation of QDs. ${ }^{135}$ Moreover, cadmium telluride (CdTe) QDs are widely used because CdTe crystals are more stable, particularly under high temperature, maintaining high crystalline purity (i.e., with minimal defects), thus providing high yield of fluorescence. ${ }^{136-138}$ Several turn-off fluorescent probes based on QDs for FA detection will be discussed as follows.
By the layer-by-layer (LBL) deposition of oppositely charged CdTe QDs and poly(dimethyldiallylemmonium chloride), Su et al. ${ }^{110}$ synthesized functional quantum dots multilayer films (QDMF). Then, bovine serum albumin was deposited on the outermost surface of the CdTe QDs multilayers by electrostatic interaction between the carboxyl groups of CdTe QDs and the amino group of bovine serum albumin. Due to the combination of electron-hole pairs between the amino group and QDs, QDMF exhibited fluorescence emission effect. When FA was adsorbed on the surface of the CdTe QDs, the excitons in them could be dissociated by transferring electrons to the acceptor (carbonyl group of FA), preventing the normal binding of electrons and holes in the QDs, which leads to turn-off fluorescence. Therefore, the concentration of FA gas can be determined by the total fluorescence decay rate and quenching intensity of the CdTe QDs. The sensing film showed an LDL of $1 \mathrm{ppb}$ toward FA with a linear range of 5-500 ppb. Moreover, due to the existence of the amino group in the outermost layer of QDMF, it also showed good selectivity for FA in the presence of other interferences.

In addition, an RGB (red, green, and blue)-sensor array based on CdTe QDs has been used to identify FA more sensitively. By modifying six ligands (three amino acids, namely, glutathione (GSH), $N$-acetyl-L-cysteine ( $\mathrm{N}$-A-Cys), L-cysteine (L-Cys), and three carboxylic acids, namely, thioglycolic acid (TGA), 3-mercaptopropionic acid, and mercaptosuccinic acid) on the surface of CdTe QDs, Wu et al. ${ }^{139}$ synthesized a red emitting fluorescence sensor array. The different ligand-modified CdTe QDs showed red fluorescence quenching phenomenon in the presence of FA. When green fluorescein was introduced, the CdTe QDs-fluorescein mixed sensor exhibited a clear fluorescence color change from red to green. The red fluorescence quenching of amino QDs was due to the aldimine condensation reaction between the $-\mathrm{NH}_{2}$ group and the $-\mathrm{CHO}$ group (Fig. 22a). The quenching effect follows the order L-Cys > $\mathrm{GSH}>\mathrm{N}$-A-cys because the nucleophilicity of primary amino and $-\mathrm{CHO}$ is stronger than that of the secondary amino group. The red fluorescence quenching of carboxylic acidfunctionalized QDs was due to the hydrogen bond between the hydrogen in FA and the oxygen on the hydroxyl group (Fig. 22b). TGA-capped QDs showed heavier quenching because the surface carboxyl groups in TGA-capped QDs are closer to the QDs. As shown in Fig. 22c, according to the linear discriminant analysis dendrogram, FA, AA, propanal, benzene, and ammonia can be distinguished within the $95 \%$ confidence interval. The sensor array also had an LDL of 80 ppb toward FA, which can meet the detection standard of WHO.

Besides, in order to distinguish the intensity of the fluorescence change more clearly and reduce the measurement error, some groups have carried out extensive research on fluorescence enhancement. For example, Xing et al. ${ }^{111}$ reported a rapid, sensitive, and highly convenient reproducible surface plasmon-enhanced optical sensor by depositing the mixture of surfactant (amino)-modified CdSe@ZnS QDs (Fig. 22d), fumed silica, and $\mathrm{Au}$ NPs with a diameter of $20 \mathrm{~nm}$ on the surface of the silica sphere array. The increase in the fluorescence 

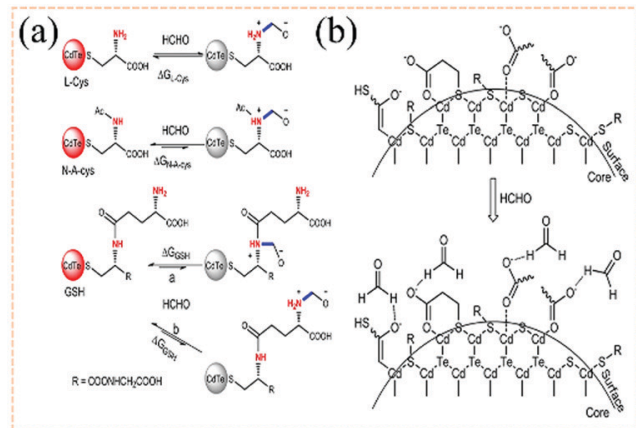

(d)

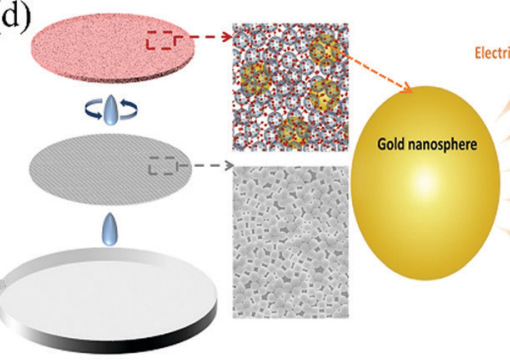

(c)

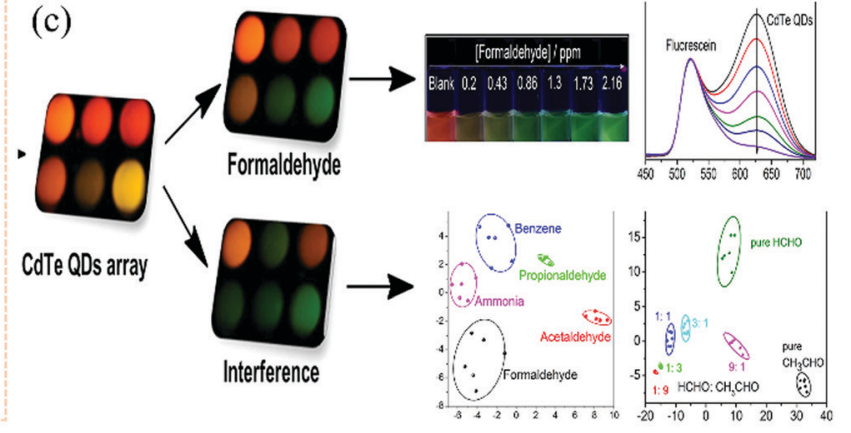

(e)

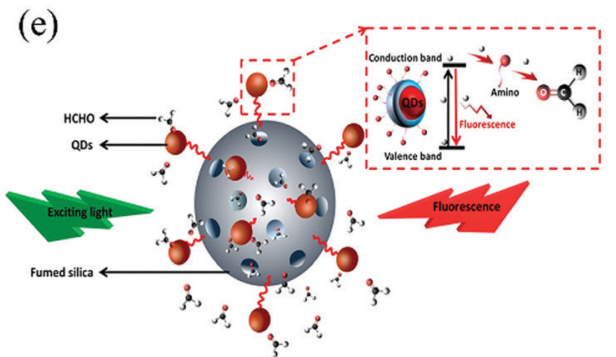

Fig. 22 (a) Possible interaction pathways for FA with L-Cys, N-A-cys, and GSH-capped CdTe QDs, (b) possible interaction pathways for FA with TGA, 3-mercaptopropionic acid, and mercaptosuccinic acid-capped CdTe QDs, (c) schematic illustration of the process for sample analysis and discrimination (Reproduced with permission. ${ }^{139}$ Copyright: 2016, Springer Nature). (d) Schematic of the fabrication process of the QDs/fumed silica/Au NPs sensing film along with the magnified cartoons of the Au NPs, fumed silica, and QD nanoparticles, and the SEM image of the silica sphere array, and (e) the schematic of the fluorescence quenching mechanism (reproduced with permission. ${ }^{111}$ Copyright: 2020, American Chemical Society).

baseline is due to the introduction of Au NPs because the LSPR of noble metal nanoparticles usually enhances the fluorescence intensity of the QDs through plasma-coupled emission or localfield enhancement (Fig. 22d). The sensing film was fabricated by the layer-by-layer method, and the self-aggregation of QDs adsorbed on the fumed silica surface significantly reduced due to electrostatic force, and the fluorescence quenching caused by AIE effect reduced. The sensing mechanism was similar to the one mentioned above; the carbonyl group of FA as the electron acceptor hindered the recombination of electrons and holes in the QDs (Fig. 22e). Besides, with the increase in the LSPR excitation efficiency of the Au NPs, a deeper and faster quenching process could be observed. The chemosensors showed excellent response (100 s) and recovery (80 s) time with an LDL of $500 \mathrm{ppb}$. It also showed excellent stability after exposure to open air for 10 days.

In addition to the above-discussed QDs based on inorganic semiconductor materials, the nanoparticles based on carbon materials, namely, carbon dots (CDs), have also been developed into fluorescence sensors, taking advantage of the CDs such as chemical robustness and surface flexibility for chemical modification. One challenge of using CDs in fluorescence sensors is the intrinsically low yield of fluorescence emission. Nonetheless, this technical drawback can be overcome by the surface passivation or doping of heteroatoms, which have been proven to be effective in improving the fluorescence yield of CD. Yang et al. ${ }^{140}$ reported a fluorescent sensor based on the structure of $\mathrm{CDs@SiO} \mathrm{SH}_{2}-\mathrm{NH}_{2}-\mathrm{Ag}^{+}$loaded onto nanosponge pieces for FA detection. Fluorescent $\mathrm{CDs@SiO}{ }_{2}-\mathrm{NH}_{2}$ was prepared by the encapsulation of CDs within the silica spheres, followed by modification with amino groups. Such aminemodified surface has strong chelation with $\mathrm{Ag}^{+}$to form $\mathrm{CDs} @ \mathrm{SiO}_{2}-\mathrm{NH}_{2}-\mathrm{Ag}^{+}$. On the basis of the silver mirror reaction, surface $\mathrm{Ag}^{+}$can be reduced by $\mathrm{FA}$ to silver nanoparticles (AgNPs), which in turn results in a significant enhancement

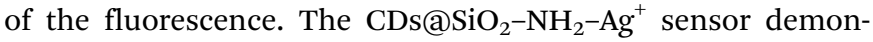
strated excellent selectivity toward FA, which is attributed to the specific silver mirror reaction between $\mathrm{Ag}^{+}$and FA. Linear dynamic range from 10 to $1000 \mathrm{ppb}$ was obtained for the sensor as the fluorescence enhancement was tested as a function of the gas concentration of FA. One technical drawback of this sensor is the long reaction (response) time, typically in the range of a few tens of minutes or hours. Under a reaction time of $30 \mathrm{~min}$, the LDL of $3 \mathrm{ppb}$ was determined.

Using fluorescent CDs, a luminescent transparent wood (LTW) was developed as the chemosensor for the real-time detection of FA gas in the gas phase. ${ }^{141}$ LTW was fabricated by encapsulating multicolor lignin-derived CDs and poly(vinyl alcohol) (PVA) into a delignified wood framework. The composite thus fabricated exhibited tunable room-temperature phosphorescence, and ratiometric fluorescence emission, in which both can be used for sensor response modulation upon interaction with FA, which in turn causes emission quenching. The ratiometric fluorescence and delayed phosphorescence sensor response gave a detection limit of 0.6 and $45 \mathrm{ppm}$, respectively.

A special kind of $\mathrm{CD}$, graphene quantum dots (GQDs), has also attracted increasing attention due to the unique electronic and optical properties of graphene intrinsic to the 2D $\pi$-comjugation. ${ }^{142}$ Wang et al. ${ }^{143}$ synthesized $\mathrm{N}$-doped graphene quantum dots (NGQDs) encapsulated by organic species, which 
exhibited $22.9 \%$ quantum yield of fluorescence, which is for conducive to development as a fluorescence sensor. Fluorescence emission was found to be capable of switching "on-off", via redox reactions with chemicals such as FA. As tested with FA, the fluorescence intensity modulation demonstrated a good linear relationship in the low concentration range of FA, with an LDL of $4.5 \mathrm{ppb}$. The NGQDs sensor was also proven to be selective toward FA.

\subsection{Functional metal-organic frameworks (MOFs)}

Unlike the QDs mentioned above, MOFs are unique crystalline porous materials with a large specific surface area, tunable pore size, and multiple functional groups. MOFs could be selfassembled by coordination bonds between the metal (ions or clusters) as nodes and functional organic ligands as supports. ${ }^{144}$ In addition, there are many kinds of fluorescence emitted by MOFs, such as organic ligand luminescence, metal-centered emission, charge transfer luminescence (ligand-to-ligand, ligandto-metal, metal-to-ligand, metal-to-metal), guest emission from lanthanide ions, fluorescent dyes encapsulated in the pores of MOFs, and guest-sensitized luminescence. The fluorescence of MOF is very sensitive to the type and concentration of analytes and to the surrounding environment, which can be changed by PET, ICT, resonance energy transfer (FRET), and competitive adsorption. ${ }^{145-148}$ Based on these unique characteristics, ${ }^{149}$ MOFs have been widely used to detect harmful gases, special air pollutants, and VOCs as ideal fluorometric gas sensing materials. ${ }^{150-153}$ In addition, lanthanide elements are usually chosen as the fluorescence center of the MOFs because of their high color purity, high quantum efficiency, and long luminescence life. ${ }^{154-156}$ Some MOF-based sensors have been reported for the sensitive detection of gaseous aldehydes.

Recently, to achieve the sensitive detection of aldehydes vapor at room temperature, Yan et al. ${ }^{157}$ reported a ligand-tometal fluorometric sensor based on $\mathrm{Eu}^{3+}$-functionalized ZnO@Uio-MOFs (precursors $\mathrm{H}_{2}$ bpydc and $\mathrm{ZrCl}_{4}$ ) heterostructures. $\mathrm{Eu}^{3+}$ could respond to the charge transfer (MOFs-to-ZnO) as a fluorescence sensing signal. The pre-adsorbed oxygen molecules were ionized (by $\mathrm{ZnO}$ ) into more active oxygen $\left(\mathrm{O}^{2-}, \mathrm{O}_{2}{ }^{-}\right)$, which would react with FA-releasing electrons. Then, the released electrons could be transferred to the excited state of $\mathrm{Eu}^{3+}$, thus enhancing the fluorescence emission of $\mathrm{Eu}^{3+}$ (Fig. 23a). A portable sensing device was fabricated by loading $\mathrm{Eu}^{3+}$-functionalized ZnO@Uio-MOFs on the test paper, which showed selectivity and sensitivity to the aldehydes with excellent LDL (42 ppb for FA, $58 \mathrm{ppb}$ for AA, and $66 \mathrm{ppb}$ for acraldehyde). The sensing mechanism was based on the signal change of the luminescence intensity, which is usually affected by the surrounding environment. In order to reduce the quantization error caused by environmental interference, Yan et al. ${ }^{158}$ subsequently reported a dual emission self-calibration and proportional light-emitting chemosensor $\left(\mathrm{Ag}^{+}-\mathrm{Eu}^{3+}\right.$ functionalized MOF) based on charge

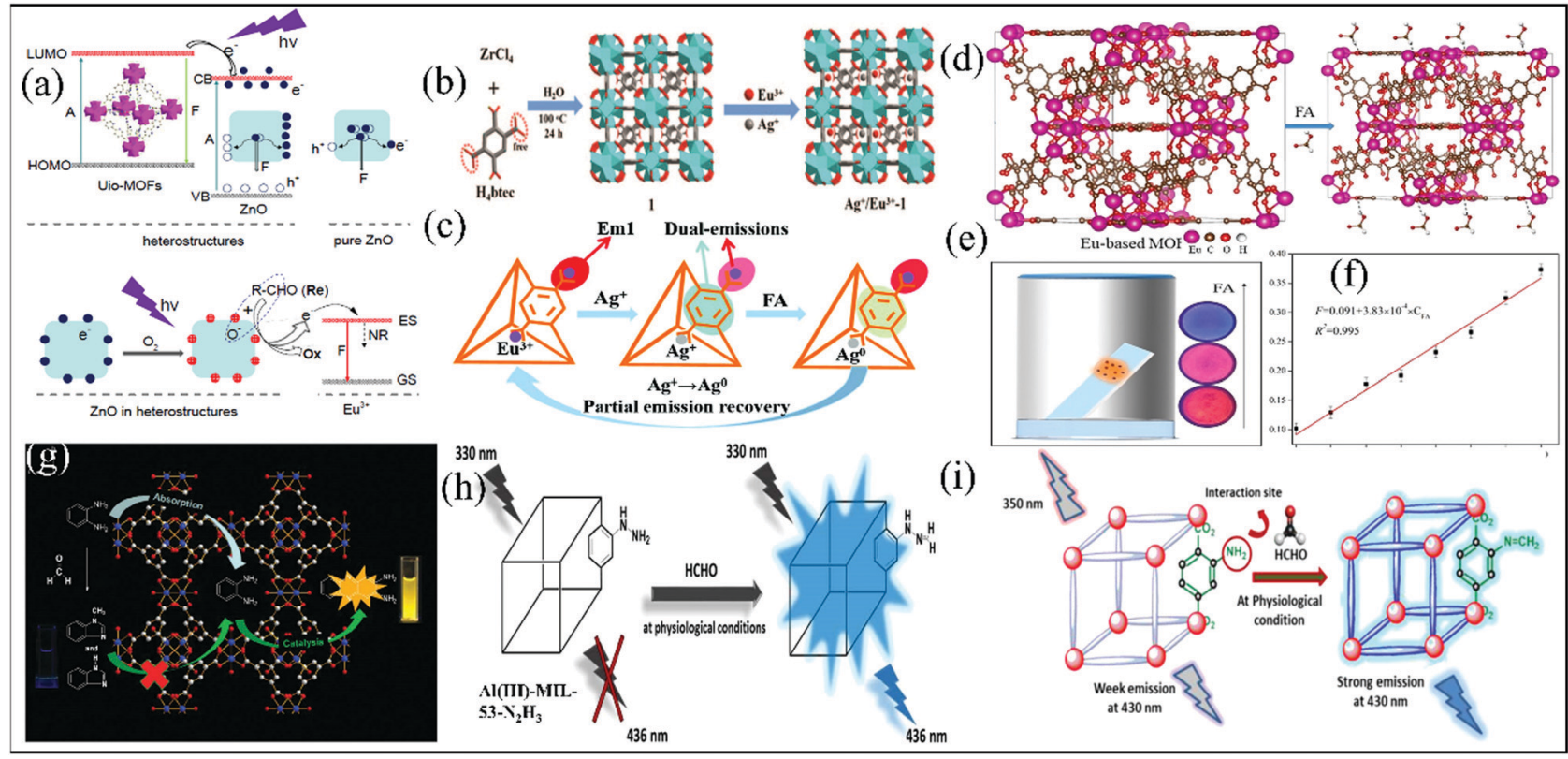

Fig. 23 (a) Schematic diagrams of MOFs-to-ZnO energy transfer in ZnO@Uio-MOFs heterostructures and the sensing mechanism toward aldehydes over $\mathrm{ZnO}$ in the heterostructures for enhanced $\mathrm{Eu}^{3+}$ based emission (reproduced with permission. ${ }^{157}$ Copyright: 2017, Royal Society of Chemistry). (b) Schematic diagram of $\mathrm{Ag}^{+}-\mathrm{Eu}^{3+}$ functionalized MOF synthesis, and (c) schematic diagrams of $\mathrm{FA}$ sensing by $\mathrm{Ag}^{+}$-Eu ${ }^{3+}$ functionalized $\mathrm{MOF}$ (Reproduced with permission. ${ }^{158}$ Copyright: 2016, Royal Society of Chemistry). (d) Schematic diagrams of Eu-based MOF in detecting FA. (e) Schematic diagram of gaseous FA detection process. (f) The changes in the fluorescence intensity ratio of Eu-based MOF with the increase in FA (0-700 ppb) (Reproduced with permission. ${ }^{159}$ Copyright: 2021, Elsevier). (g) Schematic diagrams of $\mathrm{Cu}^{2+}$-BTC MOFs for detecting the aldehydes (reproduced with permission. ${ }^{113}$ Copyright:2018, Royal Society of Chemistry). (h) Fluorogenic "turn-on" sensing of the activated Al ${ }^{3+}-\mathrm{MIL}_{-53}-\mathrm{N}_{2} \mathrm{H}_{3}$ probe at physiological conditions (reproduced with permission. ${ }^{103}$ with permission from ACS Publications). (i) Schematic diagrams of $\mathrm{Cd}^{2+}-$ based MOFs decorated with 2-amino terephthalic acid for detecting FA (Reproduced with permission. ${ }^{160}$ Copyright: 2021, American Chemical Society). 
transfer from the ligand to the metal. Moreover, the selectivity of the chemosensor to FA was increased by doping $\mathrm{Ag}^{+}$in the presence of other interfering substances (benzene, toluene and butyl acetate, ethylbenzene, etc.). Initially, fluorescence originated in the energy transfer process of the ligand $\left(\mathrm{H}_{4} \mathrm{btec}\right)$-to$\mathrm{Eu}^{3+}$. Then, with the doping of $\mathrm{Ag}^{+}$, the fluorescence of $\mathrm{Eu}^{3+}$ was gradually quenched and the emission of the functional ligand dominated due to the fact that $\mathrm{Ag}^{+}$could change the electronic energy transfer process of the lanthanide ions (Fig. 23b). Thus, the $\mathrm{Ag}^{+}-\mathrm{Eu}^{3+}$-functionalized MOF showed dual emission. When exposed to FA gas, $\mathrm{Ag}^{+}$would be reduced to $\mathrm{Ag}^{0}$ by FA, resulting in the enhanced emission of $\mathrm{Eu}^{3+}$ and the decrease in the fluorescence of organic ligands (Fig. 23c). The sensor showed good selectivity for FA with an LDL of $51 \mathrm{ppb}$ in at the room temperature.

Moreover, a fluorescent film sensor was successfully fabricated from the Eu-based MOF and tested the detection of FA in the gaseous phase (Fig. 23d). ${ }^{159}$ The Eu-based MOF was prepared by the reaction between $\mathrm{Eu}\left(\mathrm{NO}_{3}\right)_{3} 6 \mathrm{H}_{2} \mathrm{O}$ and pyromellitic acid in the presence of anhydrous sodium acetate. Upon exposure to FA, the fluorescence of the ligand increased gradually with the concentration of FA, while the characteristic emission peak of Eu showed almost no change. As a result, the emission color of the Eu-based MOF film under UV excitation changed from red to blue depending on the concentration of FA (Fig. 23e). This dramatic color change, in conjunction with the strong absorption of FA by the Eu-based MOF, makes the fabricated film an ideal sensor for the gas phase detection of $\mathrm{FA}$, which provides a linear dynamic range of $0-700 \mathrm{ppb}$ and an LDL of $11.8 \mathrm{ppb}$ for FA (Fig. 23f).

Unlike the above detection types, Shen et $a l .{ }^{113}$ synthesized a FA sensor based on $\mathrm{Cu}^{2+}$-BTC MOFs with high catalytic activity, employing 1,3,5-benzene tricarboxylic acid $\left(\mathrm{H}_{3} \mathrm{BTC}\right)$ and $\mathrm{Cu}^{2+}$ ions as the building blocks. The $\mathrm{Cu}^{2+}-\mathrm{BTC}$ MOFs could catalyze $o$-phenylenediamine (OPE) to produce the fluorometric compound (Fig. 23g). When exposed to FA vapor, the amino group of OPE reacted with FA to prevent the fluorescence emission. The probe showed good selectivity and sensitivity toward gaseous FA with an LDL of $18.67 \mathrm{ppb}$ within $60 \mathrm{~s}$. In addition, it is important to mention that $\mathrm{Cu}^{2+}$-BTC MOF can be reused at least 5 times to catalyze OPE, and its original catalytic activity will not be significantly reduced.

In addition, a new fluorescence turn-on probe $\left(\mathrm{Al}^{3+}{ }^{3+} \mathrm{MIL}-53-\right.$ $\mathrm{N}_{2} \mathrm{H}_{3}$ ) based on the PET (hydrazine to benzene ring) effect was reported by Biswas et al. ${ }^{103}$ for FA detection. The probe was composed of $\mathrm{Al}^{3+}$-MOF, containing MIL-53 (MIL = Material of Institute Lavoisier) framework topology and the hydrazinefunctionalized $\mathrm{H}_{2} \mathrm{BDC}-\mathrm{N}_{2} \mathrm{H}_{3}$ (2-hydrazinyl-1,4 benzenedicarboxylic acid) ligand, which was loaded on the quartz slide. The hydrazine functional group in the $\mathrm{H}_{2} \mathrm{BDC}-\mathrm{N}_{2} \mathrm{H}_{3}$ ligand reacts with FA to produce hydrazone, which inhibited the process of PET and resulted in a fluorescence turn-on signal (Fig. 23h). The probe had an LDL toward FA in different solvents (250 ppb in HEPES buffer, $64.33 \mathrm{ppb}$ in aqueous). Although the LDL in the mixed gas phase had not been reported, it showed selectivity toward FA in the presence of other aldehydes vapors. Similar to the $\mathrm{Al}^{3+}-\mathrm{MIL}-53-\mathrm{N}_{2} \mathrm{H}_{3}$ probe, Bej et al. ${ }^{160}$ synthesized two $\mathrm{Cd}^{2+}$-based MOFs (CMERI-1 \& CMERI-2), which can be used as an effective fluorescence turn-on sensor for detecting FA (Fig. 23i). The mechanism of fluorescence turn-on involves the blocking of the photoinduced electron transfer (from amine subunit to phenyl backbone) by the absorption of FA On fabricating the MOFs, interestingly, they used a porous hydrogel membrane, and the efficient detection of FA in the gas phase could be achieved as the substrate with the emission intensity of the film increasing gradually. The emission of the MOF film increased with the gradual exposure to FA vapor. A with an LDL of $7.8 \mathrm{ppm}$ was determined for the film sensor. Moreover, the MOF film demonstrated show high sensitivity toward the targeted analyte (FA) over other competing aldehyde analogues such as analytes (butyraldehyde, propionaldehyde, valeraldehyde, 1-napthaldehyde, etc.).

Utilizing a new chair-conformation resorcin[4]arene-based octacarboxylate ligand, Ma et al. synthesized four MOFs, which demonstrated the efficient detection of aldehydes in the gas phase. ${ }^{161}$ The sensor mechanism is based on fluorescence quenching, which in turn is due to competitive absorption between the MOFs and aldehydes, both in the wavelength range of 240-300 $\mathrm{nm}$. When the aldehydes are present in high concentration, UV excitation would be mostly absorbed by the aldehydes, leaving no excitation of the MOF, thus resulting in a decrease in the fluorescence. Particularly for benzaldehyde, which has an absorption spectrum almost overlapping with the MOF, the fluorescence quenching was found to be the strongest among all the aldehydes tested, which may lead to the selective detection of this specific aldehyde.

\section{Colorimetric/fluorometric dual-mode sensors}

Compared with the single-mode sensor, the sensor based on colorimetric and fluorometric dual-modes can reduce or eliminate the errors caused by the changes in the external environment, the instrument itself, and the parameters through the additional correction of two kinds of output signals. The detection mechanism of the dual-mode sensor toward FA detection is also based on the above-mentioned mechanism.

In 2018, a dual-mode sensor was prepared by Aquino et al. ${ }^{162}$ by immersing the silica gel plate in a dopamine solution containing sucrose and glycine. Initially, the test plate was colorless and not fluorescent. As shown in Fig. 24a, the test plate reacted with FA to form dopamine derivatives. Then, the dopamine derivatives were oxidized to tetrahydroisoquinoline, which showed fluorescence emission and yellow color (Fig. 24a). The dual-mode sensor also showed good selectivity toward FA vapor in the presence of other interfering substances such as acetone, methanol, toluene, and AA. When exposed to FA vapor for about $30 \mathrm{~min}$, a chromogenic change from white to yellow was clearly observed. However, the colorimetric LDL is 
(a)

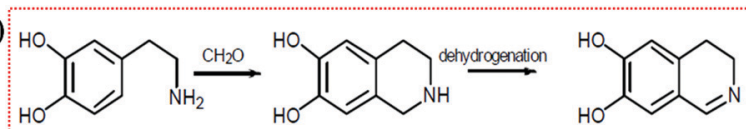

(b)

colorless, not fluorescent

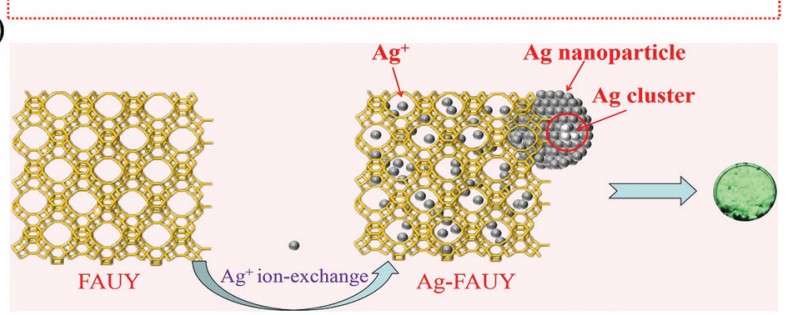

(c)

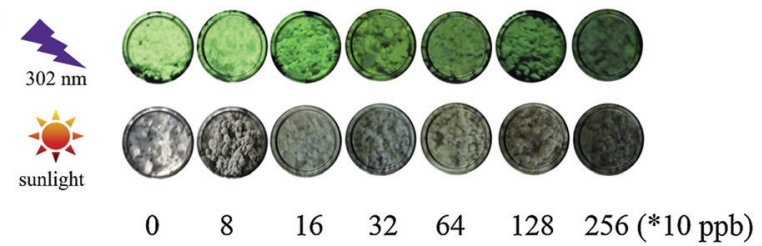

Fig. 24 (a) Schematic diagrams of the dual-mode sensor for detecting FA. $^{152}$ (b) Scheme of the luminescent mechanism of Ag-zeolites of faujasite $\mathrm{Y}$-type, and (c) digital photographs of Ag-zeolites of faujasite $\mathrm{Y}$-type at different FA concentrations (reproduced with permission. ${ }^{153}$ Copyright: 2017, Elsevier)

$700 \mathrm{ppb}$, which cannot meet the detection limit required by WHO. The sensor can also detect FA in water based on an intense fluorescence emission, and the fluorometric LDL is $0.24 \mathrm{mM}$. Therefore, the dual-mode sensor can also be used for the detection of aldehydes in different media based on different optical signals.

Taking advantage of the high selectivity and sensitivity of $\mathrm{Ag}^{+}$toward FA, a dual-mode sensor with an LDL of $750 \mathrm{ppb}$ in the presence of potentially interfering chemical substances (ethanol, ethyl acetate, benzene, etc.) was reported Li et al. ${ }^{163}$ The sensor was composed of Ag-zeolites of faujasite Y-type with bright green fluorescence, which used zeolites of faujasite Y-type $\left(\left(\mathrm{Na}^{+}{ }_{6.5}\right)\left[\mathrm{Al}_{6.5} \mathrm{Si}_{17.5} \mathrm{O}_{48}\right]\right)$ as the scaffold. When exposed to $\mathrm{FA}, \mathrm{Ag}$ clusters were formed due to the reduction of $\mathrm{Ag}^{+}$by FA, resulting in fluorescence quenching (Fig. 24b). Then, Ag clusters were oxidized to silver oxide with a detectable color change. As shown in Fig. 24c, with the increase in the FA concentration, the fluorescence was quenched and the color changed gradually.

\section{Chemiluminescent Sensors}

Chemiluminescence, an electromagnetic radiation phenomenon caused by chemical reactions, shows promising potential in the detection of certain gases owing to its independent light generation without external instruments for optical excitation system. ${ }^{164-166}$ Besides, since electronic excited states are specially determined in the process of chemiluminescence aroused by certain chemical reactions, this kind of luminescence has great advantages of high quantum yields, superior sensitivity, rapidity, safety, and controllable emission rate, which is distinct from fluorescence emission. Many research groups conducted their research to develop a new chemiluminescence-based sensor platform in the liquid phase samples during the past decades. ${ }^{167-169}$ In fact, though several kinds of light emitting mechanisms have been developed, most of them cannot be applied to gas detection readily due to the limitation of reaction environments and elevated temperature during the chemiluminescence process. Nevertheless, the attempt at the detection of reactive gas through chemiluminescence, such as nitrogen oxides $\left(\mathrm{NO}_{x}\right)^{170}$ and aldehydes, ${ }^{171}$ was developed since 1970s. With the discovery of chemical reactions among functional groups or on certain surfaces, many research groups noticed that choosing a certain compound for detecting an analyte or providing a probe on the surface to monitor the luminescence are both promising methods for gaseous aldehyde detection.

Back in 1973, Sheinson et al. ${ }^{171}$ noticed the emission spectra of electronically excited FA $\left({ }^{1} \mathrm{~A}_{2} \rightarrow{ }^{1} \mathrm{~A}_{1}\right)$ from two-stage ignition in a Vertical Tube Reactor combustion flow system. The very low intensity UV-visible emission from both the cool flame reaction zone $\left(200-400{ }^{\circ} \mathrm{C}\right)$ and the blue flame reaction zone (400-800 ${ }^{\circ} \mathrm{C}$ ) was due to FA chemiluminescence. Afterward, the reactions based on the conjugated effect and the inductive effect of the aldehyde group provide an innovative vision on gaseous aldehyde detection. For example, Maeda et al. ${ }^{172}$ developed a continuous flow method to determine gaseous FA by the reaction with hydrogen peroxide. It showed that the emission intensity of the CL reaction was proportionate to the FA concentration from $10 \mathrm{ppb}$ to $50 \mathrm{ppb}$, and the LDL was as low as $10 \mathrm{ppb}$.

To construct on-line FA monitoring systems, Zhou et al. ${ }^{173}$ designed an FA sensor using nanosized $\mathrm{V}_{2} \mathrm{Ti}_{4} \mathrm{O}_{13}$ as a sensing probe through a rapid and sensitive cataluminescence process, which is an electromagnetic radiation emission produced by catalytic oxidation reactions. As FA in air was oxidized on the surface at a certain temperature, the luminescence intensity at a certain wavelength was observed on the surface of the sensing nanomaterials $\left(\mathrm{V}_{2} \mathrm{Ti}_{4} \mathrm{O}_{13}\right)$. The result indicated high selectivity for FA at $490 \mathrm{~nm}$ and satisfactory activity at $370{ }^{\circ} \mathrm{C}$, and the LDL was about $44.78 \mathrm{ppb}$, demonstrating the feasibility of gaseous FA sensors based on chemiluminescence sensed by nanomaterials.

The process of CL is always accompanied with energy transfer, indicating potential inferior performance due to heat dissipation. Thus, designing and choosing a proper chemical reaction for the CL process affects the practical performance and application. In the future, as more reaction mechanisms of reactions will be proved and discovered by theories and experiments, and more materials doped with metal ions will be developed to detect gaseous VOCs, CL may represent another research direction for optical aldehyde sensing methods. Further research is still required to develop existing mechanisms in various environments or to design new materials to improve the sensitivity, selectivity, stability, and response time. Moreover, accessory components are necessary for designing portable devices at the same time. 


\section{Conclusion and prospects}

Aldehydes, particularly FA, have been widely used in the industry, causing serious environmental pollution and health threats. The development of quick, sensitive, and ideally lowcost sensor techniques that can detect and monitor aldehydes has become increasingly important and attracted more attention in the recent years. For small and volatile aldehydes, the detection in gas phase represents the quick and direct monitoring of the air quality. Moreover, aldehydes (e.g., FA, acetaldehyde) are also a product of the human metabolism and present in exhaled breath. Monitoring the concentration change of the aldehydes in the breath sample may lead to the quick diagnosis of diseases at an early stage. This is especially essential for cancer treatment, for which there would be more options for medical treatment, if diagnosed at an early stage. ${ }^{174}$ This is especially essential for cancer treatment, for which there would be more options for medical treatment if diagnosed at an early stage. With the rapid advancement of functional materials and structural design along with computational modeling assistance, numerous optical chemosensors have recently been developed as covered in this review. These sensors demonstrate remarkable performance in the gas phase detection of aldehydes regarding both sensitivity and selectivity. Compared to other analytical methods such as spectrophotometry and electrochemistry, optical chemosensors are usually simple to operate and are low in cost, while still remaining the same level of detection sensitivity. More importantly, because of the uniqueness of molecular design, optical chemosensors can be optimized to be selective for targeting a specific analyte. This is superior to many other detection techniques (e.g., electrochemical sensors) that often suffer from the interference of the species similar in structure or chemical property to the target analyte.

This review summarizes gaseous aldehydes' detection based on colorimetric, fluorometric, colorimetric/fluorometric dualmode, and chemiluminescence in the recent years with the comparison of their sensing performance. In particular, the selection and design of chemical sensing mechanisms and corresponding functional materials are emphasized. Different mechanisms have complex influences on the design and choice of probing materials, sensor architectures, and their fabrication, as well as performances, especially sensitivity, selectivity, and the used environment (in the solution or gas phase). Therefore, the sensing performances between sensors employing different principles cannot be arbitrarily evaluated. Indeed, a suitable optical chemosensor toward aldehyde detection should be significantly considered according to the need for special practical application.

Because of their simplicity of use, colorimetric chemosensors have great potential of application in the detection of indoor aldehydes, which enables real time monitoring of the aldehyde level by the color change even with naked eyes, etc. Using a color reader instead, the detection can be more quantitative with LDL down to the ppb level. Colorimetric sensors are normally based on reactions between the aldehyde
Table 3 Comparison between different optical chemosensors

\begin{tabular}{|c|c|c|c|}
\hline Sensor mechanism or system & Selectivity & $\begin{array}{l}\text { Interference } \\
\text { by ketone }\end{array}$ & $\begin{array}{l}\text { Interference } \\
\text { by other } \\
\text { VOCs }\end{array}$ \\
\hline Hydroxylamine acid salt & FA & $\nu$ & $x$ \\
\hline Schiff reagents & FA & $x$ & $x$ \\
\hline AHMT & FA & $x$ & $x$ \\
\hline DNPH & Aldehydes & レ & $x$ \\
\hline Amino-doped CLCs & Aldehydes & $x$ & $x$ \\
\hline Amino-functionalized CNCs & Aldehydes & $x$ & $x$ \\
\hline $\begin{array}{l}\text { Nanofibers assembled } \\
\text { from PAs }\end{array}$ & Aldehydes & $x$ & $x$ \\
\hline Sensors based on & FA & $x$ & $x$ \\
\hline \multicolumn{4}{|l|}{ Hantzsch reaction } \\
\hline Sawicki reaction & FA & $x$ & $x$ \\
\hline $\begin{array}{l}\text { Tollens' reaction- } \\
\text { mediated LSPR }\end{array}$ & Aldehydes & $x$ & $x$ \\
\hline NBD derivatives & FA, AA & $x$ & $x$ \\
\hline Naphthalimide derivatives & FA & $x$ & $x$ \\
\hline Pillar[n] arene derivatives & FA & $x$ & $x$ \\
\hline Aza-Cope rearrangement & FA & $x$ & $x$ \\
\hline QDs & FA & $x$ & $x$ \\
\hline MOFs & Aldehydes & $x$ & $x$ \\
\hline
\end{tabular}

and the sensing reagents such as hydroxylamine acid salt, DNPH reagents, amine-functionalized CNCs, and Tollens' reaction-mediated LSPR (Table 3). Since these sensing reactions have both high sensitivity and selectivity toward aldehydes, the corresponding sensors can also be potentially used for breath analysis by detecting the concentration change in the aldehydes, though a challenge may exist for distinguishing different types of aldehydes present in breath. In comparison, fluorometric sensors are usually more sensitive and thus quantitively accurate than colorimetric sensors. Most of the fluorometric sensors based on Aza-Cope rearrangement work well (regarding sensitivity and selectivity) only toward FA; thus, they are not suitable for the detection of other aldehydes. Alternatively, fluorometric sensors based on functional MOFs and QDS materials can detect both FA and other aldehydes by modifying their surface with different functional groups (Table 3). In addition to the sensitivity and selectivity, the safety and stability also have to be considered when designing new sensors for aldehyde detection. For example, silver ammonia solution used in colorimetric sensors is a strong base, corrosive, and unstable when stored under ambient conditions as it will be converted into $\mathrm{Ag}_{3} \mathrm{~N}$. Thus, sensor reagents such as silver ammonia are not suitable for use in indoor detection. In addition, the cost of the matrix material, the time consumption, and the difficulty of operation should also be considered in the practical application. The development of optical chemosensors for gaseous aldehyde detection, irrespective of whether via indirect (solution-mediated) or direct (film) analyte exposure, is flourishing and urgently required in various fields, Thus, the design and manufacturing of a sensor system with high sensitivity and selectivity as well as rapid response and optimal reversibility is still highly in need. In the future, more efforts should be invested into promoting the practical use of current sensors, designing novel functional materials relying on existing or new principles, as well as bringing new sensor fabrication 
approaches into this field. In this regard, we focus mostly on the novel molecular and structural design of optical chemosensors in this review, along with the various sensor mechanisms that enable the gas phase detection of aldehydes including FA. As FA represents the most volatile, most commonly used aldehyde compound, causing the most serious hazards and health threats, it has been studied the most extensively as the target analyte, representing the aldehydes, especially in gas phase detection. Thus, it is no surprise that FA is still dominant as the example aldehyde analyst in the research of optical chemosensors. Nevertheless, with a more comprehensive review on the sensing mechanisms and the related molecular designs, we hope to provide some design rules that may eventually help to expand the current detection capability from FA to many other aldehydes such as acetaldehyde, and butyl and pentyl aldehydes. Many of these aldehydes are proven to be effective biomarkers for the breath analysis of diseases for early-stage diagnosis.

\section{Author contributions}

CL: writing the original draft, preparing the figures, and finishing up the revisions. SF: reading and editing; MZ, RD, QT and CW: editing and revising the manuscript; SC: research and supervision of the whole review content, helping in writing and editing the original draft; LZ: supervising the whole writing process, and revising the manuscript.

\section{Conflicts of interest}

There are no conflicts to declare.

\section{Acknowledgements}

We thank the financial support of the China Scholarship Council (No. 201808360327) and the financial support from the Scientific Research Fund of Shaanxi University of Science and Technology.

\section{References}

1 H. Plaisance, P. Mocho and V. Desauziers, Indoor Air, 2020, 30, 1256-1267.

2 T. Salthammer, Data Brief, 2019, 22, 400-435.

3 D. Calestani, R. Mosca, M. Zanichelli, M. Villani and A. Zappettini, J. Mater. Chem., 2011, 21, 15532-15536.

4 D. Sun, Y. Le, C. Jiang and B. Cheng, Appl. Surf. Sci., 2018, 441, 429-437.

5 M. M. Boucher, M. H. Furigay, P. K. Quach and C. S. Brindle, Org. Process Res. Dev., 2017, 21, 1394-1403.

6 Z. Bo, M. Yuan, S. Mao, X. Chen, J. Yan and K. Cen, Sens. Actuators, B, 2018, 256, 1011-1020.

7 D. Liu, J. Wan, H. Wang, G. Pang and Z. Tang, Inorg. Chem. Commun., 2019, 102, 203-209.
8 G. D. Nielsen, S. T. Larsen and P. Wolkoff, Arch. Toxicol., 2017, 91, 35-61.

9 Z. Wang, H. Zhou, D. Han and F. Gu, J. Mater. Chem. C, 2017, 5, 3254-3263.

10 D. McGregor, H. Bolt, V. Cogliano and H. B. RichterReichhelm, Crit. Rev. Toxicol., 2006, 36, 821-835.

11 W. Miekisch, J. K. Schubert and G. F. E. NoeldgeSchomburg, Clin. Chim. Acta, 2004, 347, 25-39.

12 Z. Zhang, W. Yu, J. Wang, D. Luo, X. Qiao, X. Qin and T. Wang, Anal. Chem., 2017, 89, 1416-1420.

13 X. Qiao, B. Su, C. Liu, Q. Song, D. Luo, G. Mo and T. Wang, Adv. Mater., 2018, 30, 1702275.

14 T. Salthammer, S. Mentese and R. Marutzky, Chem. Rev., 2010, 110, 2536-2572.

15 F. F. Giubbina, C. Scaramboni, B. S. De Martinis, D. GodoySilva, R. F. P. Nogueira and M. L. A. M. Campos, Anal. Methods, 2017, 9, 2915-2922.

16 X. Nie, Z. Chen, Y. Tian, S. Chen, L. Qu and M. Fan, Food Chem., 2021, 340, 127930.

17 N. Canha, J. Lage, S. Candeias, C. Alves and S. M. Almeida, Atmos. Pollut. Res., 2017, 8, 1132-1142.

18 X. Cui, G. Fang, L. Jiang and S. Wang, Anal. Chim. Acta, 2007, 590, 253-259.

19 C. Gong, W. Huang, J. Liu, F. Wei, J. Yu, X. Si, F. Liu and Y. Li, Fuel, 2018, 221, 188-195.

20 M. Yang, M. Ospina, C. Tse, S. Toth, S. P. Caudill and H. W. Vesper, Chem. Res. Toxicol., 2017, 30, 1592-1598.

21 G. Sakai, N. Matsunaga, K. Shimanoe and N. Yamazoe, Sens. Actuators, B, 2001, 80, 125-131.

22 J. Devkota, P. R. Ohodnicki and D. W. Greve, Sensors, 2017, 17, 801.

23 D. Calestani, R. Mosca, M. Zanichelli, M. Villani and A. Zappettini, J. Mater. Chem., 2011, 21, 1553215536

24 A. Allouch, M. Guglielmino, P. Bernhardt, C. A. Serra and S. Le Calvé, Sens. Actuators, B, 2013, 181, 551-558.

25 P. R. Chung, C. T. Zeng, M. T. Ke and C. Y. Lee, Sensors, 2013, 13, 4468-4484.

26 Z. Xu, J. Chen, L. L. Hu, Y. Tan, S. H. Liu and J. Yin, Chin. Chem. Lett., 2017, 28, 1935-1942.

27 S. K. Kim, M. Gupta and H. I. Lee, Sens. Actuators, B, 2018, 257, 728-733.

28 V. Kumar, B. Maiti, M. Chini, P. De and S. Satapathi, Sci. Rep., 2019, 9, 7269.

29 S. M. Shaban, J. Y. Lee and D. H. Kim, ACS Omega, 2020, 5, 10696-10703.

30 X. Liu, N. Li, M. Li, H. Chen, N. Zhang, Y. Wang and K. Zheng, Coord. Chem. Rev., 2020, 404, 213109.

$31 \mathrm{M} . \mathrm{Xu}, \mathrm{B} . \mathrm{R}$. Bunes and L. Zang, ACS Appl. Mater. Interfaces, 2011, 3, 642-647.

32 S. Dai, N. Prempeh, D. Liu, Y. Fan, M. Gu and Y. Chang, Carbohydr. Polym., 2017, 174, 531-539.

33 T. Endo, Y. Yanagida and T. Hatsuzawa, Sens. Actuators, B, 2007, 125, 589-595.

34 J. M. Garcia, F. García, F. Serna and J. Peña, Polym. Rev., 2011, 51, 341-390. 
35 B. T. E. Thornton, A. Harrison, A. L. Pham, C. E. Castano and C. Tang, ACS Omega, 2018, 3, 3587-3591.

36 A. Ahmed, P. John, M. H. Nawaz, A. Hayat and M. Nasir, ACS Appl. Nano Mater., 2019, 2, 5156-5168.

37 X. Chen, T. Pradhan, F. Wang, J. S. Kim and J. Yoon, Chem. Rev., 2012, 112, 1910-1956.

38 X. Chen, Y. Zhou, X. Peng and J. Yoon, Chem. Soc. Rev., 2010, 39, 2120-2135.

39 H. N. Kim, W. X. Ren, J. S. Kim and J. Yoon, Chem. Soc. Rev., 2012, 41, 3210-3244.

40 X. Zhang, J. Yin and J. Yoon, Chem. Rev., 2014, 114, 4918-4959.

41 C. M. da Silva, D. L. da Silva, L. V. Modolo, R. B. Alves, M. A. de Resende, C. V. B. Martins and Â. de Fátima, J. Adv. Res., 2011, 2, 1-8.

42 N. Nakano, M. Ishikawa, Y. Kobayashi and K. Nagashima, Anal. Sci., 1994, 10, 641-645.

43 N. Nakano and K. Nagashima, J. Environ. Monit., 1999, 1, 255-258.

44 X. Wang, Y. Si, J. Wang, B. Ding, J. Yu and S. S. Al-Deyab, Sens. Actuators, B, 2012, 163, 186-193.

45 Q. Meng, T. Han, G. Wang, N. Zheng, C. Cao and S. Xie, Sens. Actuators, B, 2014, 196, 238-244.

46 J. Park, Y. Kim, C. Lee, J. W. Kook, D. Kim, J. H. Kim, K. S. Hwang and J. Y. Lee, Polymers, 2020, 12, 998.

47 B. Ding, M. Wang, X. Wang, J. Yu and G. Sun, Mater. Today, 2010, 13, 16-27.

48 X. Wang, B. Ding, J. Yu, Y. Si, S. Yang and G. Sun, Nanoscale, 2011, 3, 911-915.

49 X. Wang, B. Ding, J. Yu, M. Wang and F. Pan, Nanotechnology, 2009, 21, 055502.

50 P. J. Stoward, J. Histochem. Cytochem., 1966, 14, 681-683.

51 Y. Y. Maruo, J. Nakamura, M. Uchiyama, M. Higuchi and K. Izumi, Sens. Actuators, B, 2008, 129, 544-550.

52 X. Wang, Y. Li, X. Li, J. Yu, S. S. Al-Deyab and B. Ding, Sens. Actuators, B, 2014, 203, 333-339.

53 R. G. Dickinson and N. W. Jacobsen, J. Chem. Soc. D, 1970, 1719-1720.

54 K. Kawamura, K. Kerman, M. Fujihara, N. Nagatani, T. Hashiba and E. Tamiya, Sens. Actuators, B, 2005, 105, 495-501.

55 C. F. Lin, H. W. Zan, C. J. Lu, H. F. Meng and O. Soppera, Org. Electron., 2019, 73, 115-121.

56 X. L. Guo, Y. Chen, H. L. Jiang, X. B. Qiu and D. L. Yu, Sensors, 2018, 18, 3141.

57 Vinita, M. Tiwari, N. Agnihotri, M. Singh, A. K. Singh and R. Prakash, ACS Omega, 2019, 4, 16733-16742.

58 J. R. Askim, M. Mahmoudi and K. S. Suslick, Chem. Soc. Rev., 2013, 42, 8649-8682.

59 Y. Y. Broza, R. Vishinkin, O. Barash, M. K. Nakhleh and H. Haick, Chem. Soc. Rev., 2018, 47, 4781-4859.

60 Z. Li, J. R. Askim and K. S. Suslick, Chem. Rev., 2019, 119, 231-292.

61 J. Li, C. Hou, D. Huo, M. Yang, H. B. Fa and P. Yang, Sens. Actuators, B, 2014, 196, 10-17.

62 M. C. Janzen, J. B. Ponder, D. P. Bailey, C. K. Ingison and K. S. Suslick, Anal. Chem., 2006, 78, 3591-3600.
63 D. I. Avşar and E. Bukusoglu, Soft Matter, 2020, 16, 8683-8691.

64 D. A. Winterbottom, R. Narayanaswamy and I. M. Raimundo, Sens. Actuators, B, 2003, 90, 52-57.

65 L. Sutarlie, H. Qin and K. L. Yang, Analyst, 2010, 135, 1691-1696.

66 Y. Habibi, L. A. Lucia and O. J. Rojas, Chem. Rev., 2010, 110, 3479-3500.

67 L. Sutarlie, J. Y. Lim and K. L. Yang, Anal. Chem., 2011, 83, 5253-5258.

68 W. Song, J. K. Lee, M. S. Gong, K. Heo, W. J. Chung and B. Y. Lee, ACS Appl. Mater. Interfaces, 2018, 10, 10353-10361.

69 M. S. Gong, G. Oh, J. Chung, H. S. Jang, B. Y. Lee and W. J. Chung, Sens. Actuators, B, 2019, 282, 868-875.

70 C. K. Chang, H. L. Kuo, K. T. Tang and S. W. Chiu, Appl. Phys. Lett., 2011, 99, 073504.

71 N. Kirchner, L. Zedler, T. G. Mayerhöfer and G. J. Mohr, Chem. Commun., 2006, 1512-1514.

72 N. Tamaoki, Adv. Mater., 2001, 13, 1135-1147.

73 Y. Han, K. Pacheco, C. W. M. Bastiaansen, D. J. Broer and R. P. Sijbesma, J. Am. Chem. Soc., 2010, 132, 2961-2967.

74 J. George and S. N. Sabapathi, Nanotechnol. Sci. Appl., 2015, 8, 45-54.

75 A. Dasgupta and D. Das, Langmuir, 2019, 35, 10704-10724.

76 M. P. Hendricks, K. Sato, L. C. Palmer and S. I. Stupp, Acc. Chem. Res., 2017, 50, 2440-2448.

77 A. Dehsorkhi, V. Castelletto and I. W. Hamley, J. Pept. Sci., 2014, 20, 453-467.

78 L. Shen, S. Cao, J. Wu, J. Zhang, H. Li, N. Liu and X. Qian, Green Chem., 2009, 11, 1414-1420.

79 Y. Y. Maruo, J. Nakamura and M. Uchiyama, Talanta, 2008, 74, 1141-1147.

80 Y. Y. Maruo and J. Nakamura, Anal. Chim. Acta, 2011, 702, 247-253.

81 O. Bunkoed, F. Davis, P. Kanatharana, P. Thavarungkul and S. P. J. Higson, Anal. Chim. Acta, 2010, 659, 251-257.

82 B. J. Compton and W. C. Purdy, Can. J. Chem., 1980, 58, 2207-2211.

83 S. Mariano, W. Wang, G. Brunelle, Y. Bigay and T. H. TranThi, Procedia Eng., 2010, 5, 1184-1187.

84 M. N. Descamps, T. Bordy, J. Hue, S. Mariano, G. Nonglaton, E. Schultz, T. H. Tran-Thi and S. VignoudDespond, Sens. Actuators, B, 2012, 170, 104-108.

85 Y. Suzuki, N. Nakano and K. Suzuki, Environ. Sci. Technol., 2003, 37, 5695-5700.

86 M. L. Calvo-Muñoz, C. Roux, F. Brunet, J. P. Bourgoin, A. Ayral, A. El-Mansouri and T. H. Tran-Thi, J. Mater. Chem., 2002, 12, 461-467.

87 X. Wang, Y. Si, X. Mao, Y. Li, J. Yu, H. Wang and B. Ding, Analyst, 2013, 138, 5129-5136.

88 K. M. Mayer and J. H. Hafner, Chem. Rev., 2011, 111, 3828-3857.

89 D. Vilela, M. C. González and A. Escarpa, Anal. Chim. Acta, 2012, 751, 24-43.

90 Q. Zhang, C. Z. Huang, J. Ling and Y. F. Li, J. Phys. Chem. B, 2008, 112, 16990-16994. 
91 K. Chaiendoo, S. Sooksin, S. Kulchat, V. Promarak, T. Tuntulani and W. Ngeontae, Food Chem., 2018, 255, 41-48.

92 J. B. Zeng, S. G. Fan, C. Y. Zhao, Q. R. Wang, T. Y. Zhou, X. Chen, Z. F. Yan, Y. P. Li, W. Xing and X. D. Wang, Chem. Commun., 2014, 50, 8121-8123.

93 J. M. Lin, Y. Q. Huang, Z. B. Liu, C. Q. Lin, X. Ma and J. M. Liu, RSC Adv., 2015, 5, 99944-99950.

94 W. Duan, A. Liu, Q. Li, Z. Li, C. Y. Wen, Z. Cai, S. Tang, X. Li and J. B. Zeng, Analyst, 2019, 144, 4582-4588.

95 J. Li, Y. Wang, Q. Zhang, D. Huo, C. Hou, J. Zhou, H. Luo and M. Yang, Ana. Chim. Acta, 2020, 1096, 138-147.

96 M. Xu, J. M. Han, Y. Zhang, X. Yang and L. Zang, Chem. Commun., 2013, 49, 11779-11781.

97 F. Wang, L. Wang, X. Chen and J. Yoon, Chem. Soc. Rev., 2014, 43, 4312-4324.

98 A. Gangopadhyay, K. Maiti, S. S. Ali, A. K. Pramanik, U. N. Guria, S. K. Samanta, R. Sarkar, P. Datta and A. K. Mahapatra, Anal. Methods, 2018, 10, 2888-2894.

99 H. Ge, G. Liu, R. Yin, Z. Sun, H. Chen, L. Yu, P. Su, M. Sun, K. A. Alamry, H. M. Marwani and S. Wang, Microchem. J., 2020, 156, 104793.

100 C. Liu, A. W. Cheng, X. K. Xia, Y. F. Liu, S. W. He, X. Guo and J. Y. Sun, Anal. Methods, 2016, 8, 2764-2770.

101 K. Wei, L. Ma, G. Ma, C. Ji and M. Yin, Dyes Pigm., 2019, 165, 294-300.

102 A. Hou, H. Chen, C. Zheng, K. Xie and A. Gao, ACS Nano, 2020, 14, 7380-7388.

103 S. Nandi, E. Sharma, V. Trivedi and S. Biswas, Inorg. Chem., 2018, 57, 15149-15157.

104 Q. Lin, K. P. Zhong, J. H. Zhu, L. Ding, J. X. Su, H. Yao, T.B. Wei and Y. M. Zhang, Macromolecules, 2017, 50, 7863-7871.

105 Q. Lin, Y. Q. Fan, G. F. Gong, P. P. Mao, J. Wang, X. W. Guan, J. Liu, Y. M. Zhang, H. Yao and T. B. Wei, ACS Sustainable Chem. Eng., 2018, 6, 8775-8781.

106 Y. Zhou, J. Yan, N. Zhang, D. Li, S. Xiao and K. Zheng, Sens. Actuators, B, 2018, 258, 156-162.

107 H. Chen, Y. Zhou, K. Zheng, N. Zhang, X. Tan and W. Chen, ChemistrySelect, 2019, 4, 9622-9626.

108 X. Zhao, C. Ji, L. Ma, Z. Wu, W. Cheng and M. Yin, ACS Sens., 2018, 3, 2112-2117.

109 B. Zhai, Y. Zhang, Z. Hu, J. He, J. Liu, C. Gao and W. Li, Dyes Pigm., 2019, 171, 107743.

110 Q. Ma, H. Cui and X. Su, Biosens. Bioelectron., 2009, 25, 839-844.

111 S. Xue, X. F. Jiang, G. Zhang, H. Wang, Z. Li, X. Hu, M. Chen, T. Wang, A. Luo, H. P. Ho, S. He and X. Xing, ACS Sens., 2020, 5, 1002-1009.

112 X. Y. Xu and B. Yan, J. Mater. Chem. A, 2017, 5, 2215-2223.

113 P. Gao, X. Y. Sun, B. Liu, H. T. Lian, X. Q. Liu and J. S. Shen, J. Mater. Chem. C, 2018, 6, 8105-8114.

114 T. Mistri, R. Alam, M. Dolai, S. K. Mandal, A. R. Khuda-Bukhsh and M. Ali, Org. Biomol. Chem., 2013, 11, 1563-1569.

115 X. Wang, P. Li, W. Zhang and B. Tang, Chin. J. Anal. Chem., 2019, 47, 1537-1548.
116 M. Gao and B. Z. Tang, ACS Sens., 2017, 2, 1382-1399.

117 L. Cui, Y. Baek, S. Lee, N. Kwon and J. Yoon, J. Mater. Chem. C, 2016, 4, 2909-2914.

118 Q. Lin, Y. Q. Fan, P. P. Mao, L. Liu, J. Liu, Y. M. Zhang, H. Yao and T. B. Wei, Chem. - Eur. J., 2018, 24, 777-783.

119 L. Guo, J. Du, Y. Wang, K. Shi and E. Ma, J. Inclusion Phenom. Macrocyclic Chem., 2020, 97, 1-17.

120 J. F. Chen, G. Meng, Q. Zhu, S. Zhang and P. Chen, J. Mater. Chem. C, 2019, 7, 11747-11751.

121 X. Y. Hu, X. Liu, W. Zhang, S. Qin, C. Yao, Y. Li, D. Cao, L. Peng and L. Wang, Chem. Mater., 2016, 28, 3778-3788.

122 M. Matsumoto, R. R. Dasari, W. Ji, C. H. Feriante, T. C. Parker, S. R. Marder and W. R. Dichtel, J. Am. Chem. Soc., 2017, 139, 4999-5002.

123 K. J. Bruemmer, R. R. Walvoord, T. F. Brewer, G. BurgosBarragan, N. Wit, L. B. Pontel, K. J. Patel and C. J. Chang, J. Am. Chem. Soc., 2017, 139, 5338-5350.

124 K. J. Bruemmer, T. F. Brewer and C. J. Chang, Curr. Opin. Chem. Biol., 2017, 39, 17-23.

125 M. Chen, S. Xue, L. Liu, Z. Li, H. Wang, C. Tan, J. Yang, X. Hu, X. F. Jiang, Y. Cheng, H. Wang, X. Xing and S. He, Sens. Actuators, B, 2019, 287, 329-337.

126 S. M. Ng, M. Koneswaran and R. Narayanaswamy, RSC Adv., 2016, 6, 21624-21661.

127 S. Pany, B. Naik, S. Martha and K. Parida, ACS Appl. Mater. Interfaces, 2014, 6, 839-846.

128 R. Freeman and I. Willner, Chem. Soc. Rev., 2012, 41, 4067-4085.

129 R. Ghosh Chaudhuri and S. Paria, Chem. Rev., 2012, 112, 2373-2433.

130 B. Li, Y. Wang, L. Huang, H. Qu, Z. Han, Y. Wang, M. J. Kipper, L. A. Belfiore and J. Tang, Synth. Met., 2021, 276, 116758.

131 T. J. Deerinck, Toxicol. Pathol., 2008, 36, 112-116.

132 K. D. Wegner and N. Hildebrandt, Chem. Soc. Rev., 2015, 44, 4792-4834.

133 I. V. Martynenko, A. P. Litvin, F. Purcell-Milton, A. V. Baranov, A. V. Fedorov and Y. K. Gun'ko, J. Mater. Chem. B, 2017, 5, 6701-6727.

134 P. Wu, T. Zhao, S. Wang and X. Hou, Nanoscale, 2014, 6, 43-64.

135 M. Xia, J. Luo, C. Chen, H. Liu and J. Tang, Adv. Opt. Mater., 2019, 7, 1900851.

136 J. Kim, B. T. Huy, K. Sakthivel, H. J. Choi, W. H. Joo, S. K. Shin, M. J. Lee and Y. I. Lee, Sens. Bio-Sens. Res., 2015, 3, 46-52.

137 K. Jiang, L. Zhang, J. Lu, C. Xu, C. Cai and H. Lin, Angew. Chem., 2016, 128, 7347-7351.

138 S. M. Borisov, R. Pommer, J. Svec, S. Peters, V. Novakova and I. Klimant, J. Mater. Chem. C, 2018, 6, 8999-9009.

139 H. Xia, J. Hu, J. Tang, K. Xu, X. Hou and P. Wu, Sci. Rep., 2016, 6, 36794.

140 W. Yang, G. Zhang, J. Ni and Z. Lin, Microchim. Acta, 2020, 187, 1-8.

141 Y. Liu, H. Yang, C. Ma, S. Luo, M. Xu, Z. Wu, W. Li and S. Liu, ACS Appl. Mater. Interfaces, 2020, 12, 36628-36638. 
142 C. Xia, S. Zhu, T. Feng, M. Yang and B. Yang, Adv. Sci., 2019, 6, 1901316.

143 H. J. Li, X. Sun, F. Xue, N. Ou, B. W. Sun, D. J. Qian, M. Chen, D. Wang, J. Yang and X. Wang, ACS Sustainable Chem. Eng., 2018, 6, 1708-1716.

144 H. Y. Li, S. N. Zhao, S. Q. Zang and J. Li, Chem. Soc. Rev., 2020, 49, 6364-6401.

145 M. Pan, W. M. Liao, S. Y. Yin, S. S. Sun and C. Y. Su, Chem. Rev., 2018, 118, 8889-8935.

146 Y. Zhang, S. Yuan, G. Day, X. Wang, X. Yang and H. C. Zhou, Coord. Chem. Rev., 2018, 354, 28-45.

147 Y. Cui, Y. Yue, G. Qian and B. Chen, Chem. Rev., 2012, 112, 1126-1162.

148 W. P. Lustig, S. Mukherjee, N. D. Rudd, A. V. Desai, J. Li and S. K. Ghosh, Chem. Soc. Rev., 2017, 46, 3242-3285.

149 S. Yuan, L. Feng, K. Wang, J. Pang, M. Bosch, C. Lollar, Y. Sun, J. Qin, X. Yang, P. Zhang, Q. Wang, L. Zou, Y. Zhang, L. Zhang, Y. Fang, J. Li and H. C. Zhou, Adv. Mater., 2018, 30, 1704303.

150 M. S. Yao, J. J. Zheng, A. Q. Wu, G. Xu, S. S. Nagarkar, G. Zhang, M. Tsujimoto, S. Sakaki, S. Horike, K. Otake and S. Kitagawa, Angew. Chem., Int. Ed., 2020, 59, 172-176.

151 M. G. Campbell, D. Sheberla, S. F. Liu, T. M. Swager and M. Dincă, Angew. Chem., Int. Ed., 2015, 54, 4349-4352.

152 M. K. Smith and K. A. Mirica, J. Am. Chem. Soc., 2017, 139, 16759-16767.

153 R. W. Huang, Y. S. Wei, X. Y. Dong, X. H. Wu, C. X. Du, S. Q. Zang and T. C. W. Mak, Nat. Chem., 2017, 9, 689-697. 154 X. Y. Xu and B. Yan, J. Mater. Chem. C, 2016, 4, 8514-8521. 155 Z. Dou, J. Yu, Y. Cui, Y. Yang, Z. Wang, D. Yang and G. Qian, J. Am. Chem. Soc., 2014, 136, 5527-5530.

156 J. An, C. M. Shade, D. A. Czegan, S. Petoud and N. L. Rosi, J. Am. Chem. Soc., 2011, 133, 1220-1223.

157 Y. Zhang and B. Yan, J. Mater. Chem. A, 2017, 5, 2215-2223. 158 J. N. Hao and B. Yan, Nanoscale, 2016, 8, 12047-12053.
159 H. Che, Y. Li, X. Tian, C. Yang, L. Lu and Y. Nie, J. Hazard. Mater., 2021, 410, 124624.

160 S. Bej, S. Mandal, A. Mondal, T. K. Pal and P. Banerjee, ACS Appl. Mater. Interfaces, 2021, 13, 25153-25163.

161 H. Zhang, J. Yang, Y. Y. Liu, S. Song and J. F. Ma, Cryst. Growth Des., 2016, 16, 3244-3255.

162 C. Martínez-Aquino, A. Costero, S. Gil and P. Gaviña, Molecules, 2018, 23, 2646.

163 D. Yao, Y. Wang and H. Li, Sens. Actuators, B, 2020, 305, 127451.

164 Y. Zhu, J. Shi, Z. Zhang, C. Zhang and X. Zhang, Anal. Chem., 2002, 74, 120-124.

165 L. Q. Wu, L. C. Zhang, M. X. Sun, R. Liu, L. Z. Yu and Y. Lv, Anal. Chem., 2017, 89, 13666-13672.

166 L. Yang, G. J. Guan, S. H. Wang and Z. P. Zhang, J. Phys. Chem. C, 2012, 116, 3356-3362.

167 P. Fletcher, K. N. Andrew, A. C. Calokerinos, S. Forbes and P. J. Worsfold, Luminescence, 2001, 16, 1-23.

168 M. Liu, Z. Lin and J. M. Lin, Anal. Chim. Acta, 2010, 670, 1-10.

169 M. Ali, N. Kishikawa, K. Ohyama, H. A. Mohamed, H. AbdelWadood, A. Mahmoud, T. Imazato, Y. Ueki, M. Wada and N. Kuroda, J. Chromatogr. B: Anal. Technol. Biomed. Life Sci., 2014, 953, 147-152.

170 A. Fried, R. Sams, W. Dorko, J. W. Elkins and Z. T. Cai, Anal. Chem., 1988, 60, 394-403.

171 R. S. Sheinson and F. W. Williams, Combust. Flame, 1973, 21, 221-230.

172 Y. Maeda, X. Hu, S. Itou, M. Kitano, N. Takenaka, H. Bandow and M. Munemori, Analyst, 1994, 119, 2237-2240.

173 K. Zhou, X. Ji, N. Zhang and X. Zhang, Sens. Actuators, B, 2006, 119, 392-397.

174 J. Pereira, P. Porto-Figueira, C. Cavaco, K. Taunk, S. Rapole, R. Dhakne, H. Nagarajaram and J. S. Câmara, Metabolites, 2015, 5, 3-55. 